\title{
Insights into the Generalized Fourier's and Fick's Laws for Simulating Mixed Bioconvective Flows of Radiative-Reactive Walters-B Fluids Conveying Tiny Particles subject to Lorentz Force
}

\author{
A. Wakif \\ University of Hassan II Casablanca \\ I. L. Animasaun ( $\nabla$ ilanimasaun@futa.edu.ng ) \\ Federal University of Technology \\ Umair Khan \\ Sukkur IBA University \\ Ahmed Mohammed Alshehri \\ King Abdulaziz University
}

\section{Research Article}

Keywords: Bioconvective Walters-B nanofluid flow, Cattaneo-Christov flux, Generalized Fick's law, Arrhenius activation energy, Thermal radiation

Posted Date: April 8th, 2021

DOI: https://doi.org/10.21203/rs.3.rs-398087/v1

License: (c) (1) This work is licensed under a Creative Commons Attribution 4.0 International License.

Read Full License 


\title{
Insights into the Generalized Fourier's and Fick's Laws for Simulating Mixed Bioconvective Flows of Radiative-Reactive Walters-B Fluids Conveying Tiny Particles subject to Lorentz force
}

\author{
A.Wakif ${ }^{1 *}$, I. L. Animasaun ${ }^{2}$, Umair Khan ${ }^{3}$, Ahmed Mohammed Alshehri ${ }^{4}$ \\ ${ }^{1 *}$ Faculty of Sciences Aïn Chock, University Hassan II, Casablanca, Morocco \\ ${ }^{2}$ Fluid Dynamics and Survey Research Group, Department of Mathematical Sciences, Federal University of \\ Technology, Akure P.M.B. 704, Nigeria \\ ${ }^{3}$ Department of Mathematics and Social Sciences, Sukkur IBA University, Sukkur 65200, Sindh Pakistan \\ ${ }^{4}$ Mathematics Department, Faculty of Science, King Abdulaziz University, Jeddah 21521, Saudi Arabia \\ 1*abderrahim.wakif-etu@etu.univh2c.ma, 2ilanimasaun@futa.edu.ng, ${ }^{3}$ umairkhan@iba-suk.edu.pk, \\ 4amalshehre@kau.edu.sa
}

\begin{abstract}
The current improvement in nanoscience and nanotechnology areas has attracted researchers' attention to biofuel, bioengineering, and biomedical and mechanical engineering applications. However, there is no report on the extension of Buongiorno's model incorporating the Cattaneo-Christov theory and the generalized Fick's law to reflect the significant impacts of Brownian motion, thermophoresis diffusion, thermal radiation, and activation energy. The governing partial differential equations (PDEs) suitable to model the case as mentioned above were converted into a unified set of ordinary differential equations (ODEs) by applying appropriate similarity transformations and solved numerically by using the Spectral Local Linearization Method (SLLM) and MATLAB in-built package. The SLLM numerical method provides robustness results with a higher level of exactness and low-computational cost. It is worthy to conclude that the nanoparticles concentration distribution can be heightened considerably either by diminishing the Prandtl number and concentration relaxation parameter or increasing the values of nanoparticles concentration Biot number and activation energy parameter. An attractive reduction in the surface drag force coefficient is achievable via the intensifying values of the non-Newtonian parameter.
\end{abstract}

Keywords: Bioconvective Walters-B nanofluid flow; Cattaneo-Christov flux; Generalized Fick's law; Arrhenius activation energy; Thermal radiation

\section{Background Information}

Significant attempts have been made recently on nanotechnological topics due to nanofluids' remarkable thermodynamic uses, such as in cooling the engines of vehicles, air conditioners, highflux devices, washing machines, microwave ovens, crystal manufacturing, and numerous welding systems. Besides, the nano-engineering sector's significant advances have opened up the possibility of using magnetized nanomaterials to treat brain tumors, pharmacological treatments, artificial heart surgery, artificial lungs, and cancer therapy. Advanced nanotechnology has proposed some useful tools focused on nanomaterials' interaction to boost fossil fuel consumption and mitigate environmental crises. In this favor, the nanofluids can be utilized in a broad range of industries from transport to power generation and electronic devices, for example in transistors, silicon chip, computer processors, and micro-electro-mechanical systems as well as in the bioengineering and nanotechnology areas. Choi and Eastman [1] first introduced the nanofluid notion in 1995 as a nonhomogeneous medium. This new kind of mixtures has been received considerable attention in current times. Nanoliquids are considered to be the most satisfactory future for the coolants in the industry. Moreover, the nanofluids are essentially a combination of tiny solid nanomaterials inserted in a fluid named a base fluid to increase its thermal characteristics. For this purpose, Buongiorno [2] examines two unusual slip phenomena, specifically the Brownian motion and thermophoresis influence for the enhancement of the convective rate of thermal energy transmission. 
Based on the Buongiorno's nanofluid model, Khan and Pop [3] scrutinized the boundary layer flows of nanofluids over a stretching surface. Zaydan et al. [4] exploited the non-homogenous nanofluid model to inspect numerically the importance of suction and blowing effects on the onset of magnetoconvection in a confined nanofluidic medium. Sheikholeslami and Bhatti [5] identified the impacts of nanomaterial shapes on the features of nanofluid flows via the magnetohydrodynamic forced convection mechanism. Turkyilmazoglu [6] established a closed-form arrangement for the movement of different nanomaterials through a curved vertical surface. Bhatti and Rashidi [7] investigated the Brownian process and heat diffusion in the motion of tangent hyperbolic nanofluids submerged in a porous medium. Hayat et al. [8] analyzed the double diffusion effect of second-grade nanoliquids across a stretched sheet. In another investigation, Hayat et al. [9] examined the movement of nanofluids distracted in a Darcy-Forchheimer porous medium. The micropolar nanofluid flows with multidimensional characteristics have been numerically investigated by Hsiao [10] by considering the viscous dissipation and magnetic field effects. Gireesha et al. [11] inspected the Hall influences in two-phase flows of dusty nanofluids. More interesting works with various geometries and physical constraints are recently being done on Newtonian and non-Newtonian convective fluid/nanofluid boundary layer flows by eminent researchers in Refs. [12-18].

The inquiry of non-Newtonian fluids has a vital significance because of their widespread engineering and technological implementations. Such fluidic mediums are employed in food manufacturing, oil processing, fiber technology, material handling, nuclear industries, and many more. For instance, these liquids include ketchup, shampoos, mud, crystal manufacturing, blood, milk, paints, wire binding, grease, and so on. Several rheological models were suggested in the literature to examine the features of non-Newtonian substances, like Jeffrey fluids, power-law fluids, Sisko fluids, Casson fluids, Walters' B fluids, Oldroyd-B fluids, Eyring-Powell fluids, and many other fluids obeying non-Newtonian rheological constitutive equations. The motion of these NonNewtonian fluids has recently gained substantial attention due to their miscellaneous practical applications in vital scientific disciplines. The viscoelastic property of various polymeric liquids encountered in the food processing industry, biotechnology, and chemical engineering can be described rheologically by the Walters' B fluid model. After deriving the constitutive equations of state describing the Walters' B fluid model by Walters [19], Beard and Walters [20] exploited appropriately the established non-Newtonian rheological law to deliberate the characteristics of viscoelastic stagnation point flows driven by a variable free-stream velocity near a horizontal surface. For other purposes, Seth et al. [21] used to finite element method to explore the significant effects of Navier's velocity slip and convective thermal boundary conditions on the radiative flow of MHD viscoelastic Walters' B fluid over a vertical elongating surface. Keeping in mind the important role of Brownian and thermophoresis diffusions on the MHD nonlinear mixed radiative-convective motion of viscoelastic nanofluids over a stretching surface, Ayub et al. [22] employed properly the Cattaneo-Christov double-diffusion model along with the Walters' liquid-B model to explore semianalytically the flow characteristics as well as the heat and mass transport features of this kind of nonNewtonian nanofluids under the combined effects of generation/absorption and Arrhenius activation energy.

Naturally, the bioconvection phenomenon occurs when the motile microorganisms swim upward, which are denser than the fluid. Additionally, the self-propelled motile microorganisms raise the concentration of the base fluid by moving in a specific direction and participating in the heat and 
mass transportations inside the mediums, like biofuels and fertilizer industrial systems. The nanofluid bioconvection examined the density stratification of the mixture by considering the significant contribution of microorganisms and solid nanoparticles in the buoyancy forces. Two types of upwelling microorganisms are commonly utilized in bioconvection research studies including the low algae and other oxytactic bacteria. Albeit they are very similar in the pattern of bioconvection but still different in their orientation frameworks Ref. [23]. Due to the asymmetrical distribution of low mass, the microorganisms swim in still water. Accordingly, when these microorganisms are in the zone of motion, the swimming path is determined by the balance between the toques outstanding to the viscous drag forces resulting from the shear flow and the buoyancy forces acting on the cell. Based on these ideas, Kuznetsov and Avramenko [24] examined the bioconvection behavior of nanofluid flows containing solid nanomaterials and motile microorganisms. Besides, Kuznetsov [25] discussed the nanofluid bioconvection phenomenon for water-based suspensions involving nanomaterials and microorganisms. Nayak et al. [26] considered several slipping impacts on the there-dimensional flow of chemically reactive Casson fluids through an exponentially stretching sheet. Kumar et al. [27] scrutinized numerically the heat and mass transfer features of 3D bioconvective nanofluid flow over a horizontal slender sheet in the presence of velocity slip impacts and a non-uniform internal heat sink/source along with the thermophoresis phenomenon and Brownian motion of nanoparticles. Beg et al. [28] employed CSCM (Chebyshev spectral collocation method) to scrutinize the von Karman swirling bioconvective rotating nanofluid flow with Gyrotactic microorganisms embedded in permeable medium with Stefan blowing and slip conditions.

Al-Khaled et al. [29] deliberated the bioconvection flow of tangential hyperbolic nanofluids in the presence of chemical reaction, motile microorganisms, and thermal radiation. Shukla et al. [30] considered the no similar bioconvective nanofluid flow pat an elongating sheet with nonlinear thermal radiation and inlined magnetic field effects for the entropy minimization analysis by employing HAM (Homotopy Analysis Method). Mohamed et al. [31] illustrated the effects of bioconvection flows in a horizontal channel by utilizing the Adomian decomposition technique. Naz et al. [32] discussed energetically the evolution of the MHD dynamic of cross-nanofluids with gyrotactic motile microorganisms. By adopting another non-Newtonian rheological model, Naz et al. [33] evaluated the entropy generation in stratified Walters-B nanofluids with the presence of swimming gyrotactic microorganisms. Otherwise, Tlili et al. [34] inspected the significance of activation energy and Wu's slip effect on the bioconvection flows of Oldroyd-B fluids past a cylinder. Abbas et al. [35] scrutinized the behavior of EMHD nanofluid flow through a Riga Plate in the presence of motile microorganisms. Bhatti et al. [36] studied the features of bioconvection occurrence within nanofluid flows across a stretching cylinder embedded in a porous medium. Shahid et al. [37] investigated gyrotactic microorganisms' motion within MHD nanofluid flows through a porous medium via Chebyshev spectral collocation method. The influence of bioconvection flows containing oxytactic microorganisms inside a porous square cavity had been investigated by Balla et al. [38]. Khan et al. [39] proposed a numerical model to examine the natural bioconvection flows of water-based nanofluids past a truncated cone with the existence of gyrotactic microorganisms. The behavior of nanofluid flows with gyrotactic mixed bioconvection through a circular cylinder had been provided by Rashad et al. [40]. The flows of Maxwell fluids developed over a stretched surface with gyrotactic microorganisms, and homogeneous/heterogeneous reaction had been offered by Sohail et al. [41]. 
Motivating by the above-cited research works, the MHD mixed bioconvection phenomenon and the thermal radiation and activation energy effects have been investigated comprehensively through the laminar flow of Walters-B nanofluid over a vertical linearly stretching sheet by considering the Buongiorno's model and convective boundary conditions. In this respect, the Cattaneo-Christov double diffusions were used to scrutinize the behavior of thermal and mass diffusions of solid nanoparticles. Mathematically, the nonlinear differential system of coupled partial differential equations (PDEs) modeled the present viscoelastic nanofluid flow problem was converted appropriately to a highly nonlinear boundary value problem of ordinary differential equations (ODEs) via suitable transformations, which was tackled numerically with the help of the spectral local linearization method (SLLM). Besides, the SLLM results were explained adequately via various tables and graphs.

\section{Mathematical and Physical Description}

Let us introduced a two-dimensional MHD mixed bioconvective laminar flow of an enhanced Walters-B nanofluid with the presence of motile microorganisms and linear thermal radiation as schematically drawn in Fig. 1.

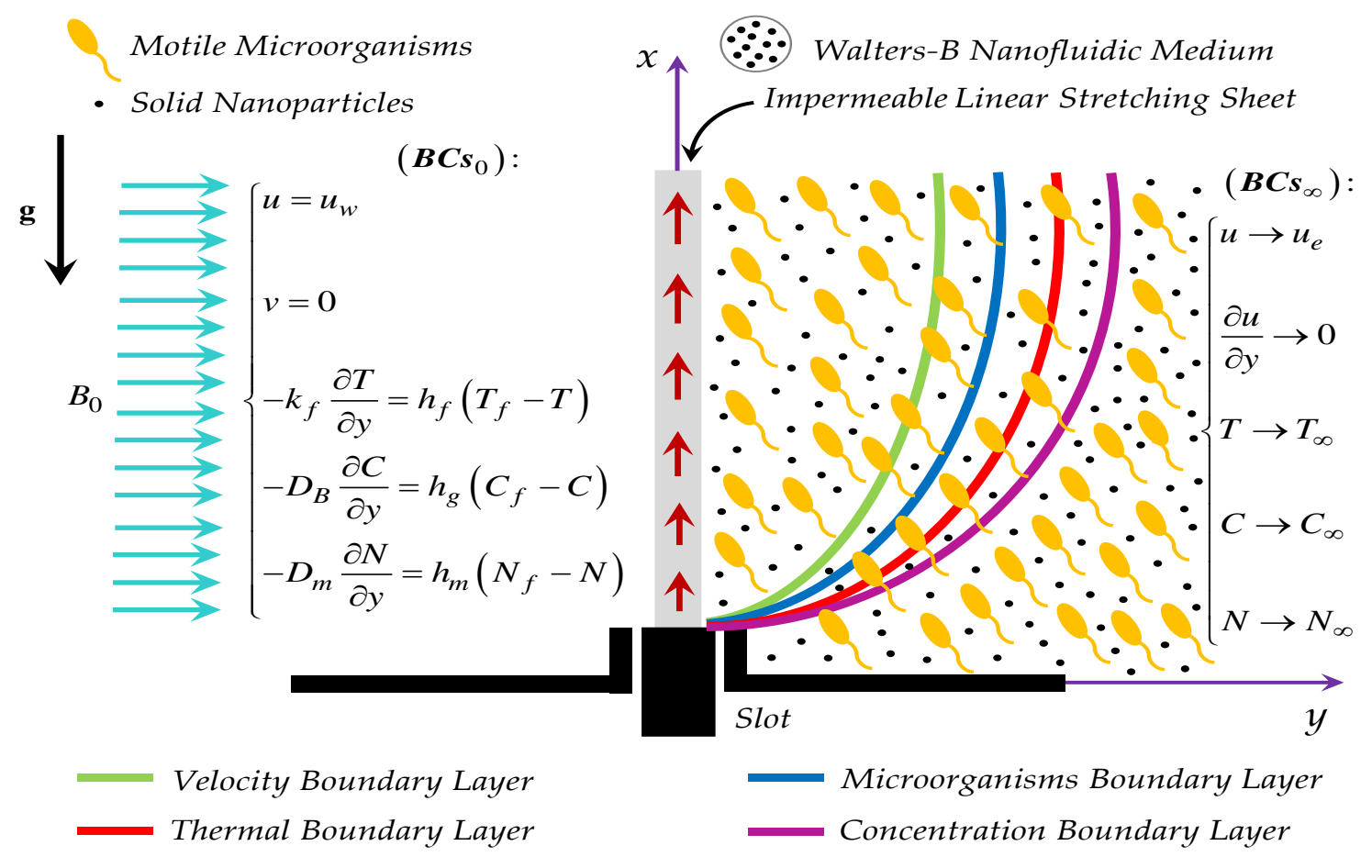

Fig.1: Geometrical configuration of the present non-Newtonian flow model.

The features of the present stagnation point flow are examined numerically by considering its motion over a vertical impermeable stretching sheet under the combined impacts of the magnetic and gravity fields of constant strengths $B_{0}$ and $g$, respectively, in which the external magnetic field is applied vertically on the sheet as underlined graphically in the geometrical configuration of the studied viscoelastic nanofluid flow. As physical constraints, multiple convective boundary conditions are exerted locally on the sheet in such a way that the convective quantities $T_{f}, C_{f}$, and $N_{f}$ characterizing the hot working fluid present a linear space-dependent in their expressions, where $T_{f} \neq T_{w}, C_{f} \neq C_{w}$, and $N_{f} \neq N_{w}$. In this inspection, the established Walters-B nanofluid flow is controlled through the 
significant interaction between the free-stream velocity $u_{e}$ and the stretching wall velocity $u_{e}$, which vary linearly in the $x$-direction. In the framework of the above considerations and the assumption of negligible magnetic Reynolds number, the present MHD mixed radiative-bioconvective viscoelastic nanofluid flow model is governed by the following conservation equations:

$$
\begin{gathered}
\frac{\partial u}{\partial x}+\frac{\partial v}{\partial y}=0, \\
u \frac{\partial u}{\partial x}+v \frac{\partial u}{\partial y}=v \frac{\partial^{2} u}{\partial y^{2}}-\frac{k_{0}}{\rho}\left(u \frac{\partial^{3} u}{\partial x \partial y^{2}}+v \frac{\partial^{3} u}{\partial y^{3}}+\frac{\partial u}{\partial x} \frac{\partial^{2} u}{\partial y^{2}}-\frac{\partial u}{\partial y} \frac{\partial^{2} u}{\partial x \partial y}\right)+u_{e} \frac{d u_{e}}{d x}-\frac{\sigma_{e} B_{0}^{2}}{\rho}\left(u-u_{e}\right)+ \\
\frac{1}{\rho}\left\{(\rho \beta) g\left(T-T_{\infty}\right)-\left[\frac{M_{p}\left(\rho_{p}-\rho_{f}\right) g}{\rho_{p}}\right]\left(C-C_{\infty}\right)-\gamma\left(\rho_{m}-\rho_{f}\right) g\left(N-N_{\infty}\right)\right\}, \\
u \frac{\partial T}{\partial x}+v \frac{\partial T}{\partial y}+\delta_{E}\left(u \frac{\partial u}{\partial x} \frac{\partial T}{\partial x}+v \frac{\partial v}{\partial y} \frac{\partial T}{\partial y}+u^{2} \frac{\partial^{2} T}{\partial x^{2}}+v^{2} \frac{\partial^{2} T}{\partial y^{2}}+2 u v \frac{\partial^{2} T}{\partial x \partial y}+u \frac{\partial v}{\partial x} \frac{\partial T}{\partial y}+v \frac{\partial u}{\partial y} \frac{\partial T}{\partial x}\right)= \\
\left(\frac{k_{f}}{\left(\rho C_{P}\right)}+\frac{16 \sigma^{*} T_{\infty}^{3}}{3 k^{*}\left(\rho C_{P}\right)}\right) \frac{\partial^{2} T}{\partial y^{2}}+\tau\left(\frac{D_{B} M_{p}}{\rho_{p}} \frac{\partial C}{\partial y}+\frac{D_{T}}{T_{\infty}} \frac{\partial T}{\partial y}\right) \frac{\partial T}{\partial y}, \\
u \frac{\partial C}{\partial x}+v \frac{\partial C}{\partial y}+\delta_{C}\left(u \frac{\partial u}{\partial x} \frac{\partial C}{\partial x}+v \frac{\partial v}{\partial y} \frac{\partial C}{\partial y}+u^{2} \frac{\partial^{2} C}{\partial x^{2}}+v^{2} \frac{\partial^{2} C}{\partial y^{2}}+2 u v \frac{\partial^{2} C}{\partial x \partial y}+u \frac{\partial v}{\partial x} \frac{\partial C}{\partial y}+v \frac{\partial u}{\partial y} \frac{\partial C}{\partial x}\right)= \\
D_{B} \frac{\partial^{2} C}{\partial y^{2}}+\frac{\rho_{p} D_{T}}{M_{p} T_{\infty}} \frac{\partial^{2} T}{\partial y^{2}}-K r^{2}\left(C-C_{\infty}\right)\left(\frac{T}{T_{\infty}}\right)^{n} \frac{\partial x p\left(\frac{-E_{a}}{k_{B} T}\right),}{u N}+v \frac{\partial N}{\partial y}+\frac{b W_{c}}{\left(C_{f}-C_{\infty}\right)} \frac{\partial N}{\partial y} \frac{\partial C}{\partial y}+\frac{b_{c} W_{c}}{\left(C_{f}-C_{\infty}\right)} N \frac{\partial^{2} C}{\partial y^{2}}=D_{m} \frac{\partial^{2} N}{\partial y^{2}} .
\end{gathered}
$$

These partial differential equations (PDEs) are supported by the following boundary conditions:

$$
\begin{aligned}
& u=u_{w}, v=0,-k \frac{\partial T}{\partial y}=h_{f}\left(T_{f}-T\right),-D_{B} \frac{\partial C}{\partial y}=h_{g}\left(C_{f}-C\right),-D_{m} \frac{\partial N}{\partial y}=h_{m}\left(N_{f}-N\right) \text { at } y=0, \\
& u \rightarrow u_{e}, \frac{\partial u}{\partial y} \rightarrow 0, T \rightarrow T_{\infty}, C \rightarrow C_{\infty}, N \rightarrow N_{\infty} \text { as } y \rightarrow \infty,
\end{aligned}
$$

in which

$$
\left\{\left(\begin{array}{l}
u_{w} \\
u_{e}
\end{array}\right)=\left(\begin{array}{l}
a \\
b
\end{array}\right) x,\left(\begin{array}{l}
T_{f} \\
C_{f} \\
N_{f}
\end{array}\right)=\left(\begin{array}{l}
T_{\infty} \\
C_{\infty} \\
N_{\infty}
\end{array}\right)+\left(\begin{array}{l}
a_{1} \\
a_{2} \\
a_{3}
\end{array}\right) x .\right.
$$

According to Ayub et al. [22], the following alterations can be adopted in this extended problem:

$$
\zeta=\left(\frac{a}{v}\right)^{\frac{1}{2}} y, u=a x f^{\prime}(\zeta), v=-(a v)^{\frac{1}{2}} f(\zeta), \theta(\zeta)=\frac{T-T_{\infty}}{T_{f}-T_{\infty}}, \phi(\zeta)=\frac{C-C_{\infty}}{C_{f}-C_{\infty}}, \chi(\zeta)=\frac{N-N_{\infty}}{N_{f}-N_{\infty}} .
$$

By making use of the above transformations, the conservations equations along with their corresponding boundary conditions are non-dimensionalized and then reduced to the following ordinary differential equations (ODEs):

$$
f^{\prime \prime \prime}-M f^{\prime}-f^{\prime 2}+f f^{\prime \prime}-\alpha\left(2 f f^{\prime \prime \prime}-f f^{i v}-f^{\prime \prime 2}\right)+\lambda(\theta-N r \phi-N c \chi)+M D+D^{2}=0,
$$




$$
\begin{gathered}
(1+R d) \theta^{\prime \prime}+\operatorname{Pr}\left(f \theta^{\prime}-f^{\prime} \theta\right)+\operatorname{Pr}\left(N b \theta^{\prime} \phi^{\prime}+N t \theta^{\prime 2}\right)-\operatorname{Pr} \alpha_{e}\left(f^{\prime 2} \theta-f f^{\prime \prime} \theta-f f^{\prime} \theta^{\prime}+f^{2} \theta^{\prime \prime}\right)=0, \\
\phi^{\prime \prime}+\frac{N t}{N b} \theta^{\prime \prime}+\operatorname{Pr} L e\left(f \phi^{\prime}-f^{\prime} \phi\right)-\operatorname{Pr} L e \alpha_{c}\left(f^{\prime 2} \phi-f f^{\prime \prime} \phi-f f^{\prime} \phi^{\prime}+f^{2} \phi^{\prime \prime}\right)-\frac{\operatorname{Pr} L e \omega(\sigma \theta+1)^{n} \phi}{\exp \left(\frac{E}{\sigma \theta+1}\right)}=0, \\
\chi^{\prime \prime}+\operatorname{Pr} L b\left(f \chi^{\prime}-f^{\prime} \chi\right)-\operatorname{Pe}\left(\phi^{\prime} \chi^{\prime}+\phi^{\prime \prime} \chi+\delta \phi^{\prime \prime}\right)=0
\end{gathered}
$$

Similarly, the boundary conditions stated in Eq. (6) become:

$$
\begin{aligned}
& f^{\prime}=1, f=0, \theta^{\prime}=-\gamma_{1}(1-\theta), \phi^{\prime}=-\gamma_{2}(1-\phi), \chi^{\prime}=-\gamma_{3}(1-\chi) \text { at } \zeta=0, \\
& f^{\prime} \rightarrow D, f^{\prime \prime} \rightarrow 0, \theta \rightarrow 0, \phi \rightarrow 0, \chi \rightarrow 0 \text { as } \zeta \rightarrow \infty .
\end{aligned}
$$

\begin{tabular}{|c|c|c|}
\hline Parameter name & Expression & Default value \\
\hline Magnetic parameter & $M=\frac{\sigma_{e} B_{0}^{2}}{a \rho}$ & $M=16$ \\
\hline Velocity ratio parameter & $D=\frac{b}{a}$ & $D=0.1$ \\
\hline Viscoelastic parameter & $\alpha=\frac{k_{0} a}{\mu}$ & $\alpha=0.1$ \\
\hline Mixed convection parameter & $\lambda=\frac{g \beta\left(T_{f}-T_{\infty}\right)}{a u_{w}}$ & $\lambda=1$ \\
\hline Buoyancy ratio parameter & $N r=\frac{\left(\rho_{p}-\rho_{f}\right) M_{p}\left(C_{f}-C_{\infty}\right)}{(\rho \beta) \rho_{p}\left(T_{f}-T_{\infty}\right)}$ & $N r=0.1$ \\
\hline Bioconvection Rayleigh number & $N c=\frac{\gamma\left(\rho_{m}-\rho_{f}\right)\left(N_{f}-N_{\infty}\right)}{(\rho \beta)\left(T_{f}-T_{\infty}\right)}$ & $N c=0.1$ \\
\hline Thermal radiation parameter & $R d=\frac{16 \sigma^{*} T_{\infty}^{3}}{3 k^{*} k}$ & $R d=0.5$ \\
\hline Prandtl number & $\operatorname{Pr}=\frac{v\left(\rho C_{P}\right)}{k}$ & $\operatorname{Pr}=10$ \\
\hline Brownian motion parameter & $N b=\frac{\tau D_{B} M_{p}\left(C_{f}-C_{\infty}\right)}{\rho_{p} v}$ & $N b=0.1$ \\
\hline Thermophoresis parameter & $N t=\frac{\tau D_{T}\left(T_{f}-T_{\infty}\right)}{v T_{\infty}}$ & $N t=0.1$ \\
\hline Thermal relaxation parameter & $\alpha_{e}=\delta_{E} a$ & $\alpha_{e}=0.01$ \\
\hline Lewis number & $L e=\frac{k}{\left(\rho C_{P}\right) D_{B}}$ & $L e=0.7$ \\
\hline $\begin{array}{l}\text { Concentration relaxation } \\
\text { parameter }\end{array}$ & $\alpha_{c}=\delta_{C} a$ & $\alpha_{c}=0.01$ \\
\hline Chemical reaction parameter & $\omega=\frac{K r^{2}}{a}$ & $\omega=1$ \\
\hline
\end{tabular}

Further, the meanings of all physical quantities arising from the present problem are summarized in the nomenclature table, whereas the dimensionless flow parameters appeared in Eqns. (9) - (13) are enlisted properly in Table1 with their explicit expressions and chosen default values.

Table 1: List of the embedded physical parameters and their default values. 


\begin{tabular}{lll}
\hline $\begin{array}{l}\text { Temperature difference } \\
\text { parameter }\end{array}$ & $\sigma=\frac{T_{f}-T_{\infty}}{T_{\infty}}$ & $\sigma=1.2$ \\
\hline Activation energy parameter & $E=\frac{E_{a}}{k_{B} T_{\infty}}$ & $E=0.1$ \\
\hline Bioconvection Lewis number & $L b=\frac{k}{\left(\rho C_{P}\right) D_{m}}$ & $L b=1$ \\
\hline Bioconvection Peclet number & $P e=\frac{b_{c} W_{c}}{D_{m}}$ & $P e=0.1$ \\
\hline $\begin{array}{l}\text { Microorganisms concentration } \\
\text { difference parameter }\end{array}$ & $\delta=\frac{N_{\infty}}{N_{f}-N_{\infty}}$ & $\delta=0.2$ \\
\hline $\begin{array}{l}\text { Thermal Biot number } \\
\text { Banoparticles concentration }\end{array}$ & $\gamma_{1}=\frac{h_{f}}{k}\left(\frac{v}{a}\right)^{\frac{1}{2}}$ & $\gamma_{1}=3$ \\
\hline $\begin{array}{l}\text { Microorganisms concentration } \\
\text { Biot number }\end{array}$ & $\gamma_{3}=\frac{h_{g}}{D_{B}}\left(\frac{v}{a}\right)^{\frac{1}{2}}$ & $\gamma_{2}=3$ \\
\hline$D_{m}$ & $\left(\frac{v}{a}\right)^{\frac{1}{2}}$ & $\gamma_{3}=3$ \\
\hline
\end{tabular}

The physical quantities of importance in this analysis are the skin friction coefficient $S_{f x}$, the Nusselt number $N u_{x}$, the Sherwood number $S h_{x}$ and the microorganisms transportation rate coefficient $N m_{x}$. These engineering amounts are defined locally as:

$$
\begin{gathered}
S_{f x}=\frac{2}{\rho u_{w}^{2}}\left[\mu\left(\frac{\partial u}{\partial y}\right)_{y=0}-k_{0}\left(u \frac{\partial^{2} u}{\partial x \partial y}+v \frac{\partial^{2} u}{\partial y^{2}}+2 \frac{\partial u}{\partial x} \frac{\partial u}{\partial y}\right)_{y=0}\right], \\
N u_{x}=-\frac{x}{k\left(T_{f}-T_{\infty}\right)}\left(k+\frac{16 \sigma^{*} T_{\infty}^{3}}{3 k^{*}}\right)\left(\frac{\partial T}{\partial y}\right)_{y=0}, \\
S h_{x}=-\frac{x}{\left(C_{f}-C_{\infty}\right)}\left(\frac{\partial C}{\partial y}\right)_{y=0} \\
N m_{x}=-\frac{x}{\left(N_{f}-N_{\infty}\right)}\left(\frac{\partial N}{\partial y}\right)_{y=0}
\end{gathered}
$$

Based on the employed mathematical transformations and the dimensionless velocity boundary conditions defined correspondingly in Eq. (8) and Eq. (13), the engineering quantities of interest $S_{f x}$, $N u_{x}, S h_{x}$ and $N m_{x}$ can take the following reduced forms:

$$
\begin{gathered}
S_{f r}=-(1-3 \alpha) f^{\prime \prime}(0), \\
N u_{r}=-(1+R d) \theta^{\prime}(0), \\
S h_{r}=-\phi^{\prime}(0), \\
N m_{r}=-\chi^{\prime}(0),
\end{gathered}
$$

in which

$$
\begin{aligned}
& S_{f r}=-\frac{1}{2} R e_{x}^{1 / 2} S_{f x}, \\
& N u_{r}=R e_{x}^{-1 / 2} N u_{x},
\end{aligned}
$$




$$
\begin{gathered}
S h_{r}=R e_{x}^{-1 / 2} S h_{x}, \\
N m_{r}=\operatorname{Re}_{x}^{-1 / 2} N m_{x}, \\
\operatorname{Re}_{x}=\frac{u_{w} x}{v} .
\end{gathered}
$$

\section{Proposed Methodology for Numerical Solutions}

This section is devoted to describing the numerical solution methodology employed for solving the highly nonlinear differential system of Eqns. (9) - (13). For reducing the mathematical complexity of the present boundary layer flow problem, the resulting coupled ODEs are linearized properly to decouple them into a sequence of dependent linear differential subsystems by utilizing the spectral local linearization method (SLLM) as explained by Motsa [42]. After some necessary rearrangements, the simplified subsystems are discretized irregularly in a well-defined computational domain based on the Gauss - Lobatto collocation points[43-49]. After a successful spatial linearization, Eqns. (9) - (13) yield to:

$$
\begin{gathered}
a_{1} f_{r+1}^{i v}+a_{2} f_{r+1}^{\prime \prime \prime}+a_{3} f_{r+1}^{\prime \prime}+a_{4} f_{r+1}^{\prime}+a_{5} f_{r+1}=R_{f}, \\
b_{1} \theta_{r+1}^{\prime \prime}+b_{2} \theta_{r+1}^{\prime}+b_{3} \theta=R_{\theta}, \\
c_{1} \phi_{r+1}^{\prime \prime}+c_{2} \phi_{r+1}^{\prime}+c_{3} \phi_{r+1}=R_{\phi}, \\
d_{1} \chi_{r+1}^{\prime \prime}+d_{2} \chi_{r+1}^{\prime}+d_{3} \chi_{r+1}=R_{\chi},
\end{gathered}
$$

in which

$$
\begin{aligned}
& f_{r+1}^{\prime}(0)=1, f_{r+1}(0)=0, \theta_{r+1}^{\prime}(0)-\gamma_{1} \theta_{r+1}(0)=-\gamma_{1}, \phi_{r+1}^{\prime}(0)-\gamma_{2} \phi_{r+1}(0)=-\gamma_{2}, \chi_{r+1}^{\prime}(0)-\gamma_{3} \chi_{r+1}(0)=-\gamma_{3} \text {, } \\
& f_{r+1}^{\prime}\left(\zeta_{\infty}\right)=D, f_{r+1}^{\prime \prime}\left(\zeta_{\infty}\right)=0, \theta_{r+1}\left(\zeta_{\infty}\right)=0, \phi_{r+1}\left(\zeta_{\infty}\right)=0, \chi_{r+1}\left(\zeta_{\infty}\right)=0 \\
& \left\{\begin{array}{l}
a_{1}=\alpha f_{r}, \\
a_{2}=-2 \alpha f_{r}^{\prime}+1, \\
a_{3}=2 \alpha f_{r}^{\prime \prime}+f_{r}, \\
a_{4}=-2 \alpha f_{r}^{\prime \prime \prime}-2 f_{r}^{\prime}-M, \\
a_{5}=\alpha f_{r}^{i v}+f_{r}^{\prime \prime}, \\
R_{f}=-\lambda \theta_{r}+\lambda N r \phi_{r}+\lambda N c \chi_{r}+\alpha f_{r} f_{r}^{i v}+\alpha f_{r}^{\prime \prime 2}-2 \alpha f_{r}^{\prime} f_{r}^{\prime \prime \prime}+f_{r} f_{r}^{\prime \prime}-f_{r}^{\prime 2}-M D-D^{2},
\end{array}\right. \\
& \left\{\begin{array}{l}
b_{1}=-\operatorname{Pr} \alpha_{e} f_{r+1}^{2}+R d+1, \\
b_{2}=\operatorname{Pr} f_{r+1}+\operatorname{Pr} N b \phi_{r}^{\prime}+2 \operatorname{Pr} N t \theta_{r}^{\prime}+\operatorname{Pr} \alpha_{e} f_{r+1} f_{r+1}^{\prime}, \\
b_{3}=-\operatorname{Pr} f_{r+1}^{\prime}-\operatorname{Pr} \alpha_{e} f_{r+1}^{\prime 2}+\operatorname{Pr} \alpha_{e} f_{r+1} f_{r+1}^{\prime \prime}, \\
R_{\theta}=\operatorname{Pr} N t \theta_{r}^{\prime 2},
\end{array}\right. \\
& \left\{\begin{array}{l}
c_{1}=-\operatorname{Pr} L e \alpha_{c} f_{r+1}^{2}+1, \\
c_{2}=\operatorname{Pr} \operatorname{Lef}_{r+1}+\operatorname{Pr} \operatorname{Le\alpha }_{c} f_{r+1} f_{r+1}^{\prime}, \\
c_{3}=-\operatorname{Pr} L e f_{r+1}^{\prime}+\operatorname{Pr} L e \alpha_{c} f_{r+1} f_{r+1}^{\prime \prime}-\operatorname{Pr} L e \alpha_{c} f_{r+1}^{\prime 2}-\operatorname{Pr} \operatorname{Le\omega }\left(\sigma \theta_{r+1}+1\right)^{n} \exp \left(\frac{-E}{\sigma \theta_{r+1}+1}\right), \\
R_{\phi}=-\frac{N t}{N b} \theta_{r+1}^{\prime \prime},
\end{array}\right. \\
& d_{1}=1, d_{2}=\operatorname{Pr} L b f_{r+1}-P e \phi_{r+1}^{\prime}, d_{3}=-\operatorname{Pr} L b f_{r+1}^{\prime}-P e \phi_{r+1}^{\prime \prime}, R_{\chi}=P e \delta \phi_{r+1}^{\prime \prime} .
\end{aligned}
$$


Here, the asymptotical dimensionless value $\zeta_{\infty}$ corresponds to the similarity variable's infinite value $\zeta($ i.e., $\zeta \rightarrow \infty)$, while the iterative subscripts $r$ and $r+1$ indicate the preceding and present approximate assessments, respectively. Now, the above-linearized equations are tackled iteratively with the following initial solutions:

$$
\left\{\begin{array}{l}
f_{0}(\zeta)=(D-1) e^{-\zeta}+D(\zeta-1)+1 \\
\theta_{0}(\zeta)=\left(\frac{\gamma_{1}}{1+\gamma_{1}}\right) e^{-\zeta} \\
\phi_{0}(\zeta)=\left(\frac{\gamma_{2}}{1+\gamma_{2}}\right) e^{-\zeta} \\
\chi_{0}(\zeta)=\left(\frac{\gamma_{3}}{1+\gamma_{3}}\right) e^{-\zeta}
\end{array}\right.
$$

By respecting the above-stated mathematical requirements, the iterative SLLM schemes converge robustly to precise numerical values of $S_{f r}, N u_{r}, S h_{r}$ and $N m_{r}$ with an absolute precision of about $10^{-10}$ in lesser CPU time as demonstrated in Table 2. Qualitatively, this high level of accuracy is optimized to be attained just after seven successive iterations (i.e., $k=7$ ) during an acceptable computational time (i. e. , $t=0.28 s$ ), in which the residual errors $\left\|\operatorname{Res}_{f}\right\|_{\infty},\left\|\operatorname{Res}_{\theta}\right\|_{\infty},\left\|\operatorname{Re} s_{\phi}\right\|_{\infty}$ and $\left\|R e s_{\chi}\right\|_{\infty}$ are around of $10^{-8}, 10^{-9}, 10^{-9}$ and $10^{-9}$, respectively. Computed quantities are

$$
\begin{aligned}
& \left\{\begin{array}{l}
\operatorname{Res}_{f}(\zeta)=\left\{\begin{array}{l}
f_{r+1}^{\prime \prime \prime}-M f_{r+1}^{\prime}-f_{r+1}^{\prime 2}+f_{r+1} f_{r+1}^{\prime \prime}-\alpha\left(2 f_{r+1}^{\prime} f_{r+1}^{\prime \prime \prime}-f_{r+1} f_{r+1}^{i v}-f_{r+1}^{\prime \prime 2}\right)+ \\
\lambda\left(\theta_{r+1}-N r \phi_{r+1}-N c \chi_{r+1}\right)+M D+D^{2}
\end{array}\right\}, \\
\left\|\operatorname{Res}_{f}\right\|_{\infty}=\operatorname{Max}\left(\left\{\left|\operatorname{Res}_{f}\left(\zeta_{i}\right)\right|, \text { where } \zeta_{N_{T}} \leq \zeta_{i} \leq \zeta_{1} \text { and } \zeta_{i}=\frac{\zeta_{\infty}}{2}\left[\cos \left(\frac{\pi i-\pi}{N_{T}-1}\right)+1\right]\right\}\right),
\end{array}\right. \\
& \left\{\begin{array}{l}
\operatorname{Res}_{\theta}(\zeta)=\left\{\begin{array}{l}
(1+R d) \theta_{r+1}^{\prime \prime}+\operatorname{Pr}\left(f_{r+1} \theta_{r+1}^{\prime}-f_{r+1}^{\prime} \theta_{r+1}\right)+\operatorname{Pr}\left(N b \theta_{r+1}^{\prime} \phi_{r+1}^{\prime}+N t \theta_{r+1}^{\prime 2}\right)- \\
\operatorname{Pr} \alpha_{e}\left(f_{r+1}^{\prime 2} \theta_{r+1}-f_{r+1} f_{r+1}^{\prime \prime} \theta_{r+1}-f_{r+1} f_{r+1}^{\prime} \theta_{r+1}^{\prime}+f_{r+1}^{2} \theta_{r+1}^{\prime \prime}\right)
\end{array}\right\}, \\
\left\|\operatorname{Res}_{\theta}\right\|_{\infty}=\operatorname{Max}\left(\left\{\left|\operatorname{Res}_{\theta}\left(\zeta_{i}\right)\right|, \text { where } \zeta_{N_{T}} \leq \zeta_{i} \leq \zeta_{1} \text { and } \zeta_{i}=\frac{\zeta_{\infty}}{2}\left[\cos \left(\frac{\pi i-\pi}{N_{T}-1}\right)+1\right]\right\}\right),
\end{array}\right. \\
& \left\{\operatorname{Res}_{\phi}(\zeta)=\left\{\begin{array}{l}
\phi_{r+1}^{\prime \prime}+\frac{N t}{N b} \theta_{r+1}^{\prime \prime}+\operatorname{Pr} \operatorname{Le}\left(f_{r+1} \phi_{r+1}^{\prime}-f_{r+1}^{\prime} \phi_{r+1}\right)- \\
\operatorname{Pr} \operatorname{Le}_{c}\left(f_{r+1}^{\prime 2} \phi_{r+1}-f_{r+1} f_{r+1}^{\prime \prime} \phi_{r+1}-f_{r+1} f_{r+1}^{\prime} \phi_{r+1}^{\prime}+f_{r+1}^{2} \phi_{r+1}^{\prime \prime}\right)- \\
\operatorname{Pr} \operatorname{Le\omega }\left(\sigma \theta_{r+1}+1\right)^{n} \exp \left(\frac{-E}{\sigma \theta_{r+1}+1}\right) \phi_{r+1}
\end{array}\right\},\right. \\
& \left\|\operatorname{Res}_{\phi}\right\|_{\infty}=\operatorname{Max}\left(\left\{\left|\operatorname{Res}_{\phi}\left(\zeta_{i}\right)\right| \text {, where } \zeta_{N_{T}} \leq \zeta_{i} \leq \zeta_{1} \text { and } \zeta_{i}=\frac{\zeta_{\infty}}{2}\left[\cos \left(\frac{\pi i-\pi}{N_{T}-1}\right)+1\right]\right\}\right) \text {, }
\end{aligned}
$$




$$
\left\{\begin{array}{l}
\operatorname{Res}_{\chi}(\zeta)=\chi_{r+1}^{\prime \prime}+\operatorname{Pr} L b\left(f_{r+1} \chi_{r+1}^{\prime}-f_{r+1}^{\prime} \chi_{r+1}\right)-\operatorname{Pe}\left(\phi_{r+1}^{\prime} \chi_{r+1}^{\prime}+\phi_{r+1}^{\prime \prime} \chi_{r+1}+\delta \phi_{r+1}^{\prime \prime}\right), \\
\left\|\operatorname{Res}_{\chi}\right\|_{\infty}=\operatorname{Max}\left(\left\{\left|\operatorname{Res}{ }_{\chi}\left(\zeta_{i}\right)\right|, \text { where } \zeta_{N_{T}} \leq \zeta_{i} \leq \zeta_{1} \text { and } \zeta_{i}=\frac{\zeta_{\infty}}{2}\left[\cos \left(\frac{\pi i-\pi}{N_{T}-1}\right)+1\right]\right\} .\right.
\end{array}\right.
$$

Here, the symbol $N_{T}$ represents the total number of collocations points $\zeta_{i}$, where $\zeta_{N_{T}}=0$ and $\zeta_{1}=\zeta_{\infty}$. Additionally, the results of the convergence and accuracy tests summarized in Table 2 assert that there is no doubt that the spectral local linearization method (SLLM) proves its numerical flexibility and capability in solving gigantic differential systems of coupled nonlinear ODEs again. From the computational point of view, the present SLLM results are presented in a tabular form with an absolute exactness of the order of $10^{-10}$ by selecting $N_{T}=70$ and $\zeta_{\infty}=15$ in all subsequent numerical spectral analysis steps. However, those fundamental values are adjusted consistently to generate noticeable graphical displays for $f^{\prime}(\zeta), \theta(\zeta), \phi(\zeta)$, and $\chi(\zeta)$ with smooth curves. To authentic once more the correctness of the implemented numerical algorithm code, extensive comparisons of the SLLM results are carried out in Table 3 and Table 4 against those reported previously in the literature for the physical quantities $f^{\prime \prime}(0), N u_{r}$ and $S h_{r}$ by Andersson [50] and Mehryan et al. [51] as well as Wubshet and Gosa [52] in the case of isothermal and isoconcentration wall conditions. A thorough analysis of the SLLM tabular data outputted for this regard confirms that the performed SLLM algorithm leads to reasonable numerical outcomes, which lends enough reliability to the current numerical investigation.

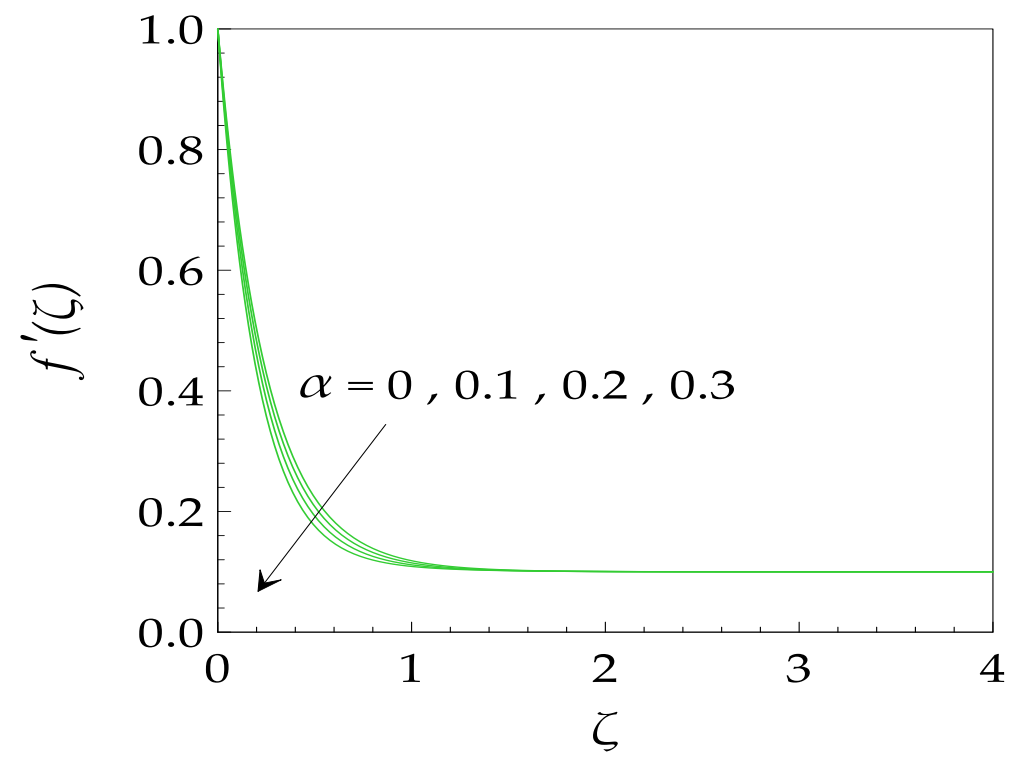

Fig.2: Profiles of $f^{\prime}(\zeta)$ at various values of $\alpha$ 
Table 2: Convergence analysis of the numerical SLLM results for the considered default parameters, when $N_{T}=70$ and $\zeta_{\infty}=15$.

\begin{tabular}{|c|c|c|c|c|c|c|c|c|c|}
\hline$k$ & $S_{f r}$ & $\left\|\operatorname{Res}_{f}\right\|_{\infty}$ & $N u_{r}$ & $\left\|R e s_{\theta}\right\|_{\infty}$ & $S h_{r}$ & $\left\|\operatorname{Res}_{\phi}\right\|_{\infty}$ & $N m_{r}$ & $\left\|\operatorname{Res}_{\chi}\right\|_{\infty}$ & CPU Time \\
\hline 1 & 2.793734656 & $8.73 \times 10^{-1}$ & 1.753283057 & $1.22 \times 10^{0}$ & 1.646593643 & $4.36 \times 10^{-8}$ & 1.558025630 & $6.81 \times 10^{-8}$ & $0.07 \mathrm{~s}$ \\
\hline 2 & 2.707620145 & $1.15 \times 10^{-2}$ & 1.713868798 & $2.19 \times 10^{-2}$ & 1.665211716 & $1.82 \times 10^{-9}$ & 1.561812108 & $7.39 \times 10^{-11}$ & $0.10 \mathrm{~s}$ \\
\hline 3 & 2.706323179 & $7.85 \times 10^{-4}$ & 1.715871199 & $1.10 \times 10^{-3}$ & 1.664713882 & $1.64 \times 10^{-9}$ & 1.561875653 & $7.09 \times 10^{-10}$ & $0.14 \mathrm{~s}$ \\
\hline 4 & 2.706425961 & $2.99 \times 10^{-5}$ & 1.715877728 & $5.69 \times 10^{-5}$ & 1.664694740 & $1.67 \times 10^{-9}$ & 1.561862479 & $7.47 \times 10^{-10}$ & $0.17 \mathrm{~s}$ \\
\hline 5 & 2.706424607 & $1.09 \times 10^{-6}$ & 1.715874856 & $2.17 \times 10^{-6}$ & 1.664695816 & $1.66 \times 10^{-9}$ & 1.561862893 & $7.66 \times 10^{-10}$ & $0.21 \mathrm{~s}$ \\
\hline 6 & 2.706424483 & $1.20 \times 10^{-7}$ & 1.715874854 & $1.28 \times 10^{-7}$ & 1.664695842 & $1.65 \times 10^{-9}$ & 1.561862903 & $7.80 \times 10^{-10}$ & $0.24 \mathrm{~s}$ \\
\hline 7 & 2.706424486 & $3.76 \times 10^{-8}$ & 1.715874860 & $4.48 \times 10^{-9}$ & 1.664695840 & $1.67 \times 10^{-9}$ & 1.561862902 & $7.71 \times 10^{-10}$ & $0.28 \mathrm{~s}$ \\
\hline 8 & 2.706424486 & $4.40 \times 10^{-8}$ & 1.715874860 & $4.23 \times 10^{-10}$ & 1.664695840 & $1.66 \times 10^{-9}$ & 1.561862902 & $7.67 \times 10^{-10}$ & $0.31 \mathrm{~s}$ \\
\hline 9 & 2.706424486 & $1.78 \times 10^{-8}$ & 1.715874860 & $4.71 \times 10^{-10}$ & 1.664695840 & $1.66 \times 10^{-9}$ & 1.561862902 & $7.67 \times 10^{-10}$ & $0.34 \mathrm{~s}$ \\
\hline 10 & 2.706424486 & $5.41 \times 10^{-8}$ & 1.715874860 & $4.53 \times 10^{-10}$ & 1.664695840 & $1.65 \times 10^{-9}$ & 1.561862902 & $7.87 \times 10^{-10}$ & $0.38 \mathrm{~s}$ \\
\hline 11 & 2.706424486 & $3.46 \times 10^{-8}$ & 1.715874860 & $4.40 \times 10^{-10}$ & 1.664695840 & $1.65 \times 10^{-9}$ & 1.561862902 & $7.69 \times 10^{-10}$ & $0.42 \mathrm{~s}$ \\
\hline 12 & 2.706424486 & $5.28 \times 10^{-8}$ & 1.715874860 & $4.69 \times 10^{-10}$ & 1.664695840 & $1.66 \times 10^{-9}$ & 1.561862902 & $7.69 \times 10^{-10}$ & $0.46 \mathrm{~s}$ \\
\hline 13 & 2.706424486 & $6.76 \times 10^{-8}$ & 1.715874860 & $4.50 \times 10^{-10}$ & 1.664695840 & $1.66 \times 10^{-9}$ & 1.561862902 & $7.66 \times 10^{-10}$ & $0.49 \mathrm{~s}$ \\
\hline 14 & 2.706424486 & $5.15 \times 10^{-8}$ & 1.715874860 & $4.47 \times 10^{-10}$ & 1.664695840 & $1.65 \times 10^{-9}$ & 1.561862902 & $7.71 \times 10^{-10}$ & $0.54 \mathrm{~s}$ \\
\hline 15 & 2.706424486 & $4.91 \times 10^{-8}$ & 1.715874860 & $4.42 \times 10^{-10}$ & 1.664695840 & $1.65 \times 10^{-9}$ & 1.561862902 & $7.76 \times 10^{-10}$ & $0.57 \mathrm{~s}$ \\
\hline
\end{tabular}


Table 3: Comparison between the SLLM numerical results and those reported previously for $f^{\prime \prime}(0)$, when $N r=N c=\lambda=D=0$.

\begin{tabular}{cccc}
\hline & & \multicolumn{2}{c}{$-f^{\prime \prime}(0)$} \\
\cline { 3 - 4 }$\alpha$ & $M$ & Ref. [50] & Present results \\
\hline 0 & 0 & 1.000000000 & 1.000000000 \\
0 & 16 & 4.123105625 & 4.123105625 \\
0.1 & 16 & 4.346134936 & 4.346134936 \\
0.2 & 16 & 4.609772228 & 4.609772228 \\
0.3 & 16 & 4.928053803 & 4.928053803 \\
0.1 & 0 & 1.054092553 & 1.054092553 \\
0.1 & 4 & 2.357022604 & 2.357022604 \\
0.1 & 9 & 3.333333333 & 3.333333333 \\
0.1 & 16 & 4.346134936 & 4.346134936 \\
\hline
\end{tabular}

Table 4: Validation of the present SLLM numerical results with those of the literature for $N u_{r}$ and $S h_{r}$, when $\operatorname{Pr}=L e=10, \quad N b=0.1, \quad$ and $\quad \alpha=N r=N c=\lambda=M=D=R d=\alpha_{e}=\alpha_{c}=\omega=P e=$ $L b^{-1}=\gamma_{1}^{-1}=\gamma_{2}^{-1}=\gamma_{3}^{-1}=0$.

\begin{tabular}{cccccccc}
\hline \multirow{3}{*}{$N t$} & \multicolumn{3}{c}{$N u_{r}$} & & \multicolumn{3}{c}{$S h_{r}$} \\
\cline { 2 - 4 } \cline { 6 - 8 } & {$[51]$} & {$[52]$} & Present results & & {$[51]$} & {$[52]$} & Present results \\
\hline 0.1 & 0.952493 & 0.95239 & 0.952366061 & & 2.129151 & 2.12938 & 2.129389070 \\
0.3 & 0.520173 & 0.52019 & 0.520290003 & & 2.528152 & 2.52855 & 2.528200344 \\
0.5 & 0.321125 & 0.32109 & 0.320507703 & & 3.034519 & 3.03511 & 3.036982888 \\
\hline
\end{tabular}

\section{Results and Discussion}

In this section, we have scrutinized the physical significance of the involved physical parameters that directly affect the nanofluid velocity distributions $f^{\prime}(\zeta)$, the nanofluid temperature $\theta(\zeta)$, the nanoparticle concentration $\phi(\zeta)$, and the concentration of the motile microorganism $\chi(\zeta)$. For this purpose, Figs. 221 are portrayed to understand better the essential features of the present MHD mixed bioconvective stagnation point flow by exploring its dynamical behavior and the characteristics of the various transport phenomena related to this study. Based on the slope linear regression method (SLRM)[5355], these interesting properties are evaluated numerically in Table 5, Table 6, Table 7, and Table 8 through the physical quantities $f^{\prime \prime}(0), S_{f r}, \theta(0), N u_{r}, \phi(0), S h_{r}, \chi(0)$ and $N m_{r}$ by examining their increasing/decreasing variations with the significant pertinent flow parameters, namely the viscoelastic parameter $\alpha$, the magnetic parameter $M$, the buoyancy ratio parameter $N r$, the bioconvection Rayleigh number $N c$, the mixed convection parameter $\lambda$, the velocity ratio parameter $D$, the Prandtl number $\operatorname{Pr}$, the thermal relaxation parameter $\alpha_{e}$, the thermal radiation parameter $R d$, the thermal Biot number $\gamma_{1}$, the concentration relaxation parameter $\alpha_{c}$, the nanoparticles concentration Biot number $\gamma_{2}$, the activation energy parameter $E$, the bioconvection Peclet number $P e$, the bioconvection Lewis number $L b$, and the microorganisms concentration Biot number $\gamma_{3}$, whose default values are quantified numerically in Table 1 unless otherwise specified. For reducing the number of the physical effects, the Brownian motion parameter 
$N b$, the thermophoresis parameter $N t$, the Lewis number $L e$, the chemical reaction parameter $\omega$, the fitted rate constant $n$, the temperature difference parameter $\sigma$, and the microorganisms concentration difference parameter $\delta$ are remained particularly fixed in this investigation at $N b=0.1, N t=0.1, L e=0.7, \omega=1$, $n=1.5, \sigma=1.2, \delta=0.2$.

\subsection{Velocity Profiles}

The variations in the velocity of the transport phenomenon reporesented by $f^{\prime}(\zeta)$ at various values of viscoelastic parameter $\alpha$, the magnetic parameter $M$, the buoyancy ratio parameter $N r$, the bioconvection Rayleigh number $N c$, the mixed convection parameter $\lambda$, and the velocity ratio parameter $D$ are shown in Figs. 2 - 8. Because of these representations, it is apparent from Figs. 2 - 5 and Fig. 8 (A, B, C, D) that an escalation in the parameters $\alpha, M, N r$, and $N c$ slows down meaningfully the nanofluid flow and shrinks the velocity boundary layer region. This decelerating trend results in a noticeable decline in the values of the curved streamlines. Physically, the damping effect of the parameter $\alpha$ can be explained more clearly by the significant upturn in the elasticity characteristic of the non-Newtonian medium, which in turn increases its specific relaxation time. Also, the slowing motion of the viscoelastic non-Newtonian nanofluid caused by the significant estimates of the parameters $M, N r$, and $N c$ is due principally to the increasingly resistive effects of the magnetic and the concentration buoyancy forces. Fundamentally, the mutual interaction between the velocity of the electrically conducting nanofluidic medium and the externally applied magnetic field leads inevitably to the so-called Lorentz forces, which are assumed of magnetic origin in the absence of an induced electrical field. However, the concentration buoyancy forces are created because of the coexistence of solid nanoparticles and motile microorganisms. These physical constraints act vertically as drag forces permitting to hinder the nanofluid motion. Differently, Fig. 6, Fig. 7, and Fig. 8 (E, F) demonstrate truly that the nanofluid velocity and the values of the streamlines increase owing to an increment in the parameters $\lambda$ and $D$. These hastening trends of the parameters $\lambda$ and $D$ are happened effectively due to the intensification in the thermal buoyancy forces and the stagnation velocity of the exterior stream, respectively. Graphically, it is noticed a slight enlargement in the velocity boundary layer thickness with the higher value of the parameters $\lambda$, while an opposite is seen for the parameter $D$, when the free-stream velocity $u_{e}$ is taken smaller that the stretching wall velocity $u_{e}$, (i.e., $\left.D<1\right)$.

\subsection{Temperature Profiles}

The nanofluid temperature behaviors versus various pertinent parameter, like the Prandtl number Pr, the thermal relaxation parameter $\alpha_{e}$, the magnetic parameter $M$, the thermal radiation parameter $R d$, and the thermal Biot number $\gamma_{1}$ are elucidated correspondingly in Figs. 9-13 via the spatial variation in the temperature solution $\theta(\zeta)$. Fig. 9 depicts the effect of the parameter Pr on the nanofluid temperature throughout the thermal boundary layer region. Here, it noticed a perceptible drop in the nanofluid temperature. This falling off in the nanofluid temperature occurs essentially due to the sudden weakness in the nanofluidic medium's thermal diffusion mechanism. Similar behavior is seen in Fig. 10 for reducing the thermal boundary layer thickness when the parameter Pr gets larger values. This is because of the decline in the parameter $\alpha_{e}$, in which the thermal boundary layer is thinner with the increasing values of the parameter $\alpha_{e}$. Unlike of the fundamental Fourier's law, the Cattaneo-Christov theory incorporates a thermal relaxation time $\delta_{E}$ in the heat transfer mechanism. Therefore, the fluid particles require a temporal delay to 
communicate an amount of their stored heat to the adjacent particles (i.e., solid nanoparticles and motile microorganisms), which produces a diminishment in the nanofluid temperature with an improvement in the modified wall temperature gradient $-\theta^{\prime}(0)$. Fig. 11 highlights that the nanofluid temperature is an increasing function of the magnetic parameter $M$. The enhancing thermal trend observed for the magnetic parameter $M$ is happened generally due to the supplementary heat arising from the resistive influence of the Lorentz forces on the nanofluid motion. Fig. 12 delineates the variation of the temperature profile $\theta(\zeta)$ in response to an increasing adjustment in the values of the thermal radiation parameter $R d$. The results of this graphical display evident that the escalating values of the thermal radiation parameter $R d$ tend to make the thermal boundary layer much thicker with a substantial upsurge in the temperature of the nanofluidic medium because of the enhancement in the apparent thermal diffusivity, which also includes the effective contribution of the radiation heat transfer mechanism. The influence of the Newtonian heating imposed at the stretching sheet is epitomized clearly in Fig. 13 via an appropriate adjustment associated with the thermal Biot number $\gamma_{1}$. In this graphical presentation, it is evidenced that the wall temperature as well as the nanofluid temperature inside the thermal boundary layer region growth gradually with the strengthening values of the parameter $\gamma_{1}$. This thermal enhancement is justified mainly by the weakening in the convective resistance at the vertical sheet, which leads to a significant heat transfer from the stretching sheet to the adjacent nanofluidic medium.

\subsection{Nanoparticles Concentration Profiles}

In this subsection, we examine the impact of some pertinent parameters involved in the nanoparticles conservation equation on the spatial distribution of the nanoparticles concentration $\phi(\zeta)$ within the nanofluidic medium. By exploiting the numerical data illustrated graphically in Figs. 14-17 for sundry values of the Prandtl number $\operatorname{Pr}$, the concentration relaxation parameter $\alpha_{c}$, the nanoparticles concentration Biot number $\gamma_{2}$, and the activation energy parameter $E$. Straightforwardly, it is remarked from Fig. 14 that the parameter Pr exhibits the same sort of effect on the nanoparticles concentration profile $\phi(\zeta)$ as previously explained for the temperature distribution $\theta(\zeta)$ in Fig. 9. According to Ghalambaz et al. [56], the concentration boundary layer region's shrinking tendency with the continuous increment in the parameter Pr can be explicated by the nanoparticles migration from the vicinity of the stretching sheet towards the free-stream region. Consequently, a certain number of solid nanoparticles are pulled out of the concentration boundary layer region due to the temperature decrease. Another similar aspect is illustrated in Fig. 15 for the impact of the parameter $\alpha_{c}$ on the nanoparticle concentration profile. This deterioration in the nanoparticles concentration field is happened mainly because of the augmentation in the concentration relaxation time $\delta_{C}$. So, the solid nanoparticles necessitate more time to diffuse randomly through the nanofluidic medium via the Brownian and thermophoresis processes. For this reason, the nanoparticles concentration profile decays with the larger estimates of the parameter $\alpha_{c}$. On the contrary, Fig. 16 discloses that the nanoparticles concentration field can be boosted substantially by specifying higher values for the nanoparticles concentration Biot number $\gamma_{2}$. This mass feature is explained physically by the decrease in the resulting convective resistance at the stretching sheet. In this situation, the nanofluid concentration becomes higher because many solid nanoparticles are pushed in the nanofluidic medium across the stretching sheet. Further, the rise in the concentration equation's generative chemical reaction equation creates a sharp deterioration in the nanoparticle concentration field. However, the effect of this 
sink term can be lessened enormously by damping the chemical reaction mechanism via the higher values of the parameter $E$ as emphasized in Fig. 17.

\subsection{Motile Microorganisms Concentration Profiles}

Sequel to the aforestated findings, further interesting physical insights are concluded from Figs. 18-21 concerning the present bioconvective phenomenon. These illustrations are exposed fittingly to pursue the concentration evolution $\chi(\zeta)$ of motile microorganisms with the magnifying values of the Prandtl number $\operatorname{Pr}$, the bioconvection Péclet number $P e$, the bioconvection Lewis number $L b$, and the microorganisms concentration Biot number $\gamma_{3}$. The graphical findings elucidated for the parameter Pr in Fig.18 demonstrate that the motile microorganisms promote the less viscous medium. Consequently, the microorganisms boundary layer region becomes thinning with the parameter Pr's augmenting values due to the partial migration of motile microorganisms to the free-stream region. Similarly, an eventual decrease in the concentration of the motile microorganism with the amplifying values of the parameters $P e$ and $L b$ is discerned in Fig.19 and Fig.20, respectively. From a dynamical point of view, this declining influence of the parameters $P e$ and $L b$ is due primarily to the sudden descent in the coefficient $D_{m}$ characterizing the diffusive motion of motile microorganisms. An opposite behavior was observed and illsutarted in Fig.21 for the motile microorganisms concentration profile against the enhancing values of the parameter $\gamma_{3}$. As similarly explained before, the reason behind the resulting growth in the concentration of motile microorganisms with the higher estimates of the parameter $\gamma_{3}$ is justified by the decline in the bioconvective resistance at the stretching sheet. In this case, many motile microorganisms are brought quickly to the nanofluidic medium via the limiting wall.

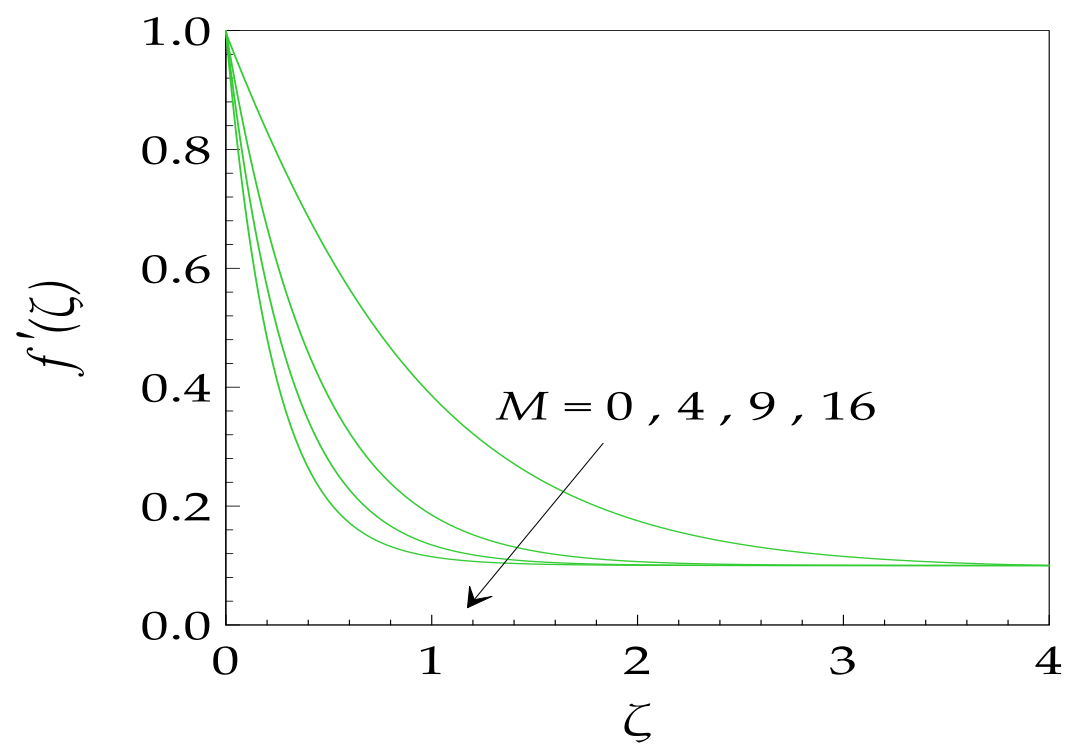

Fig.3: Profiles of $f^{\prime}(\zeta)$ at various values of $M$. 


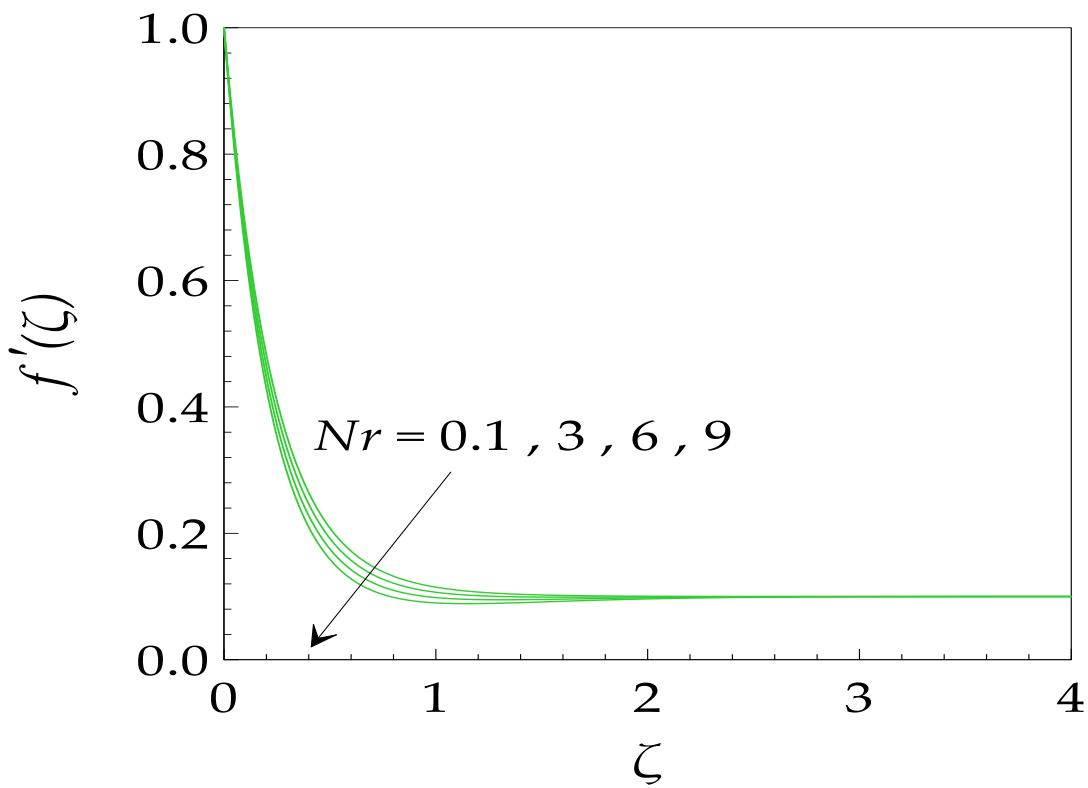

Fig.4: Profiles of $f^{\prime}(\zeta)$ at various values of $N r$.

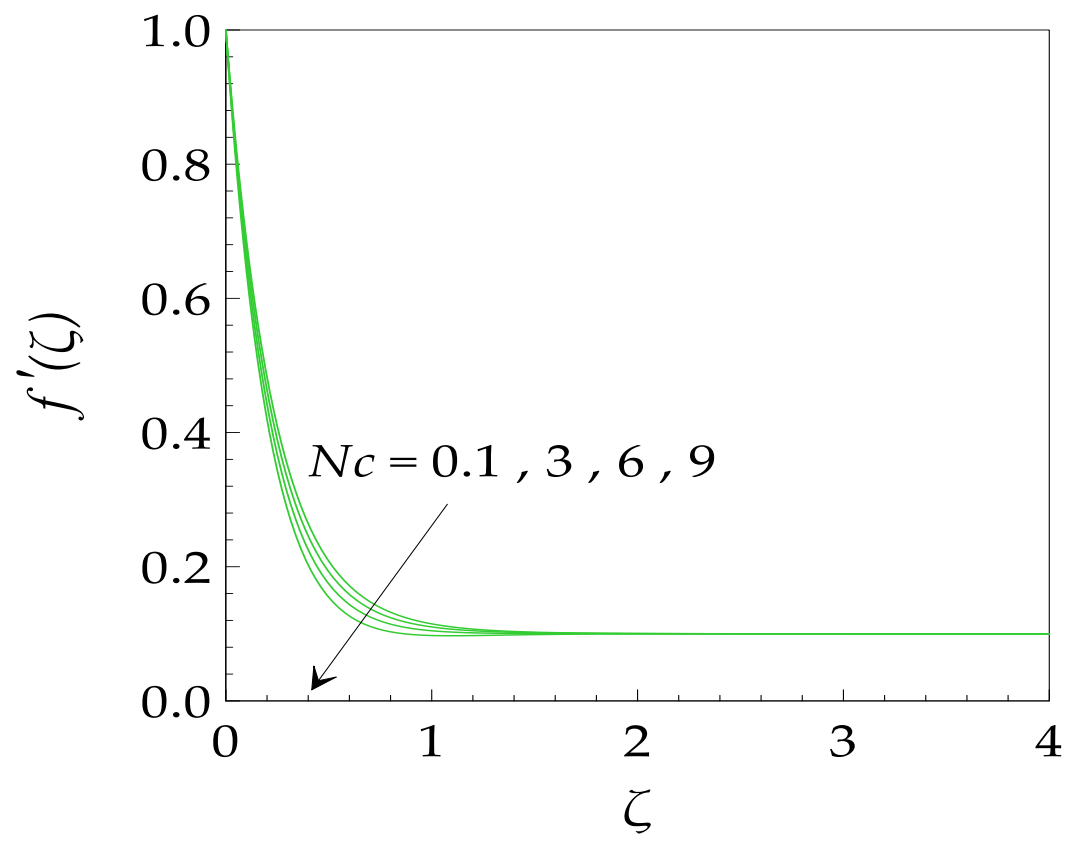

Fig.5: Profiles of $f^{\prime}(\zeta)$ at various values of $N c$. 


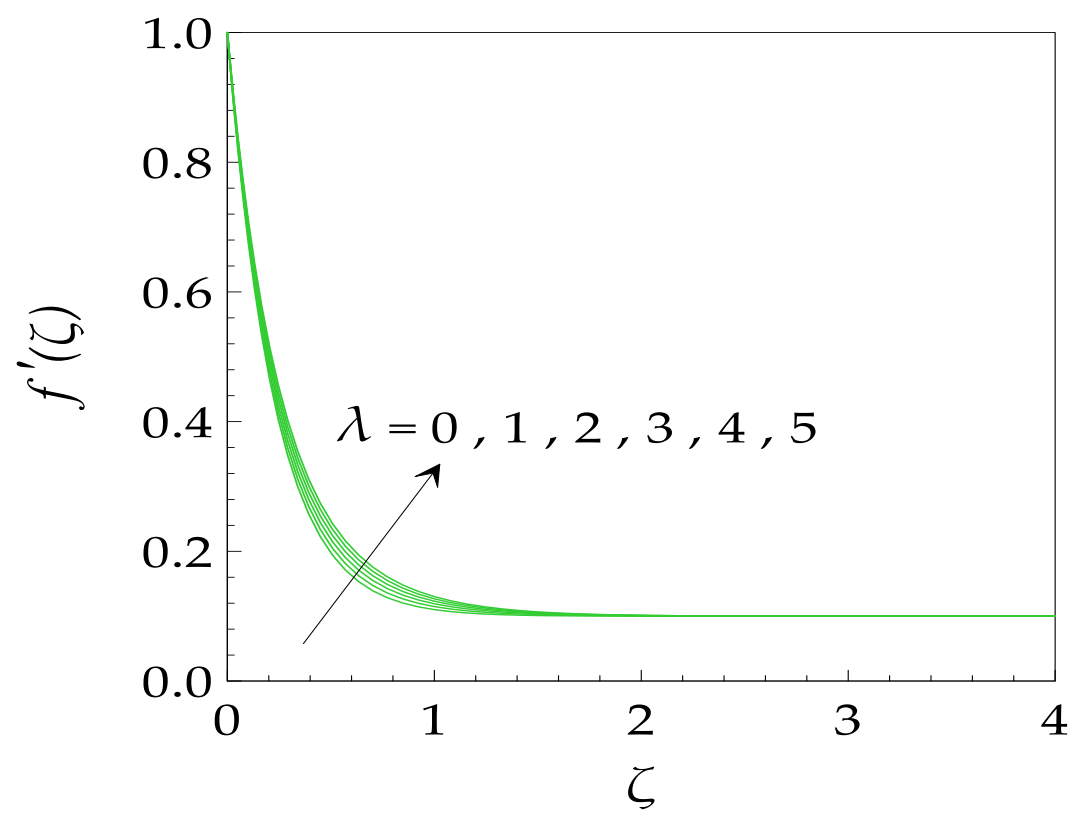

Fig.6: Profiles of $f^{\prime}(\zeta)$ at various values of $\lambda$.

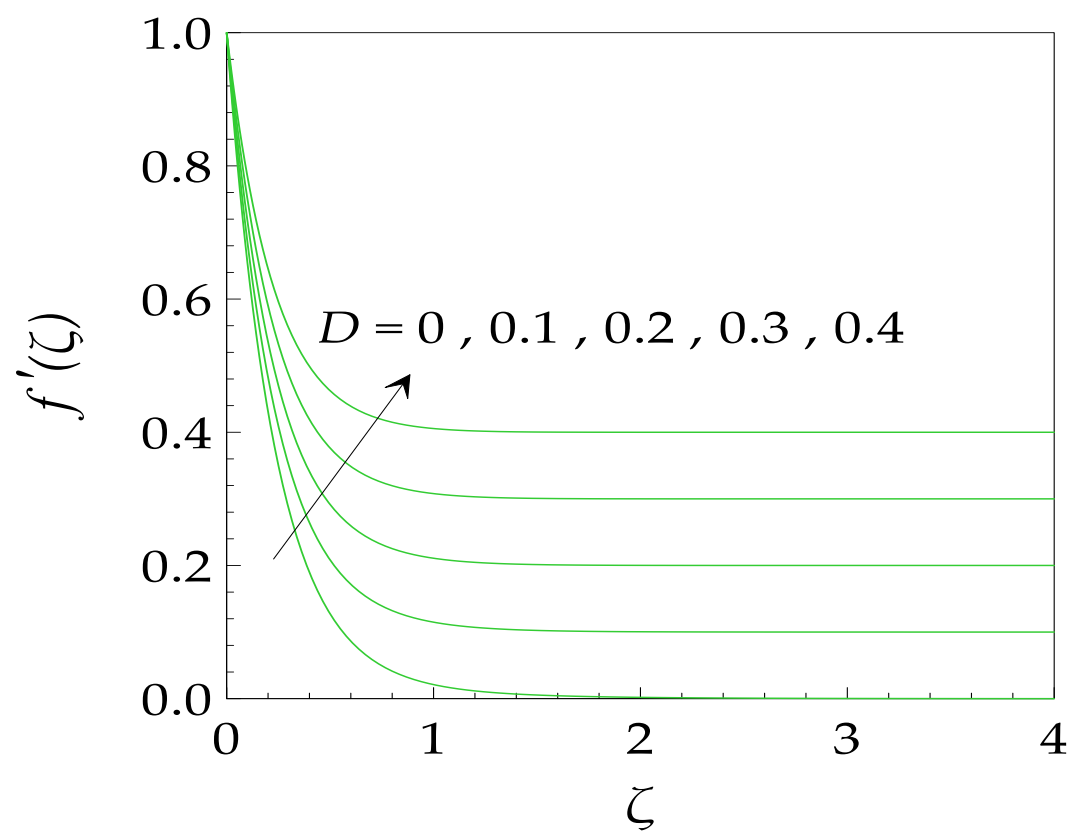

Fig.7: Profiles of $f^{\prime}(\zeta)$ at various values of $D$. 


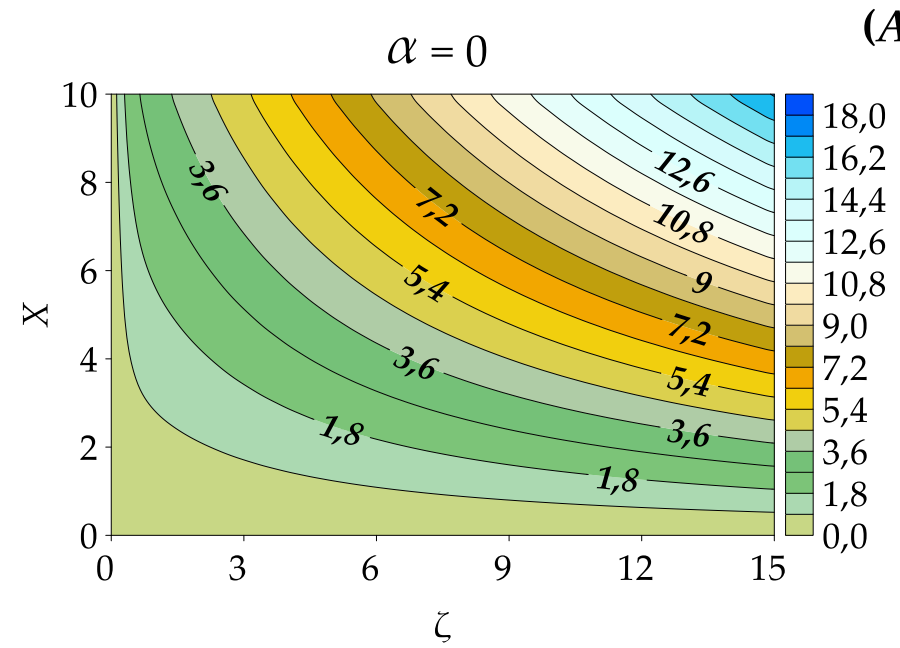

(A)
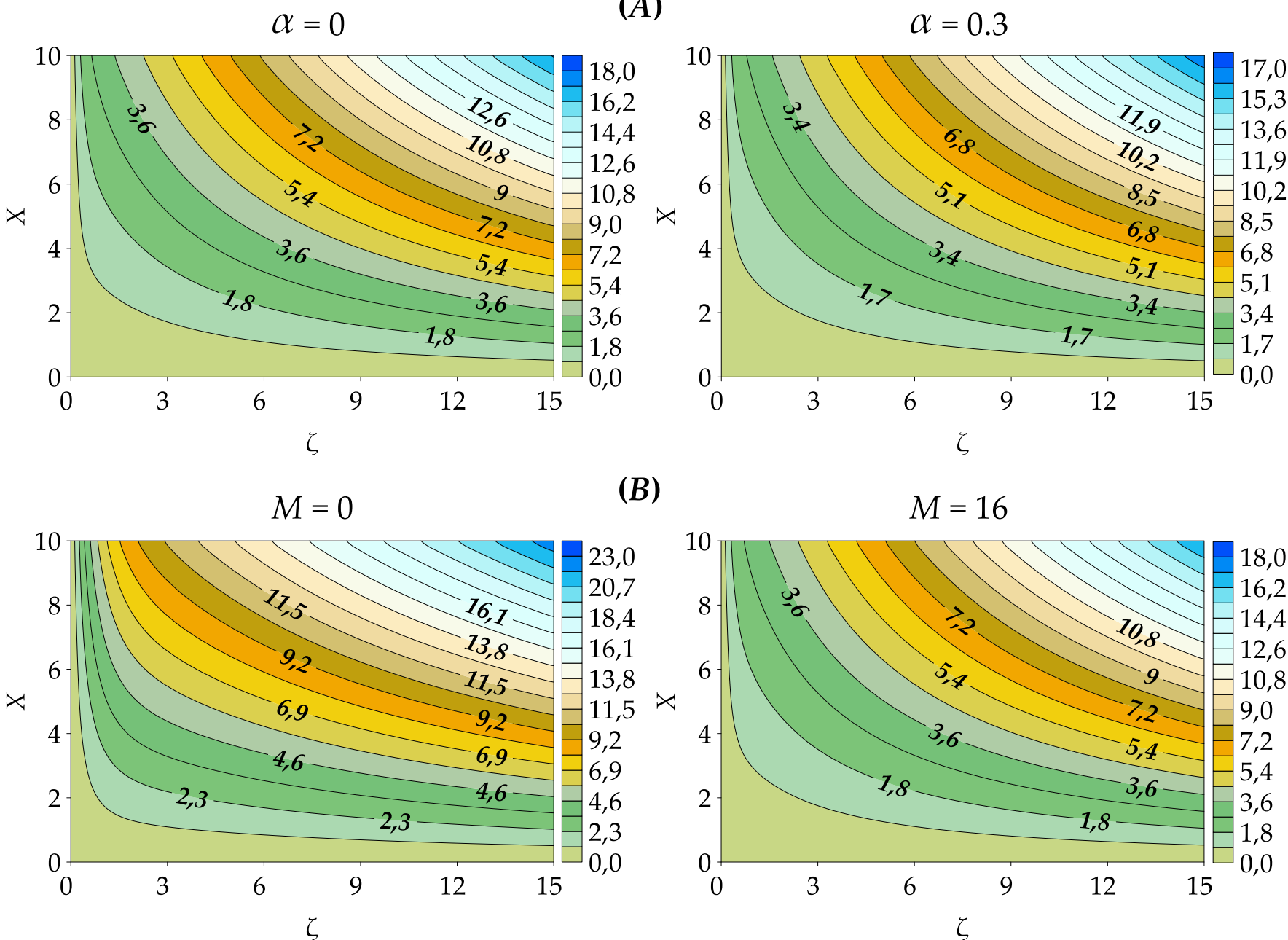

(B)

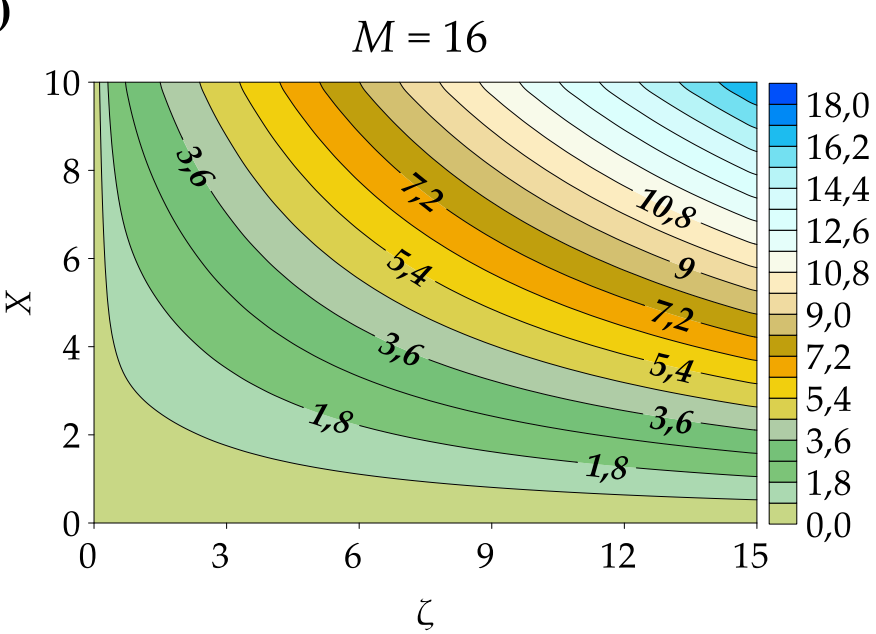

$N r=0.1$

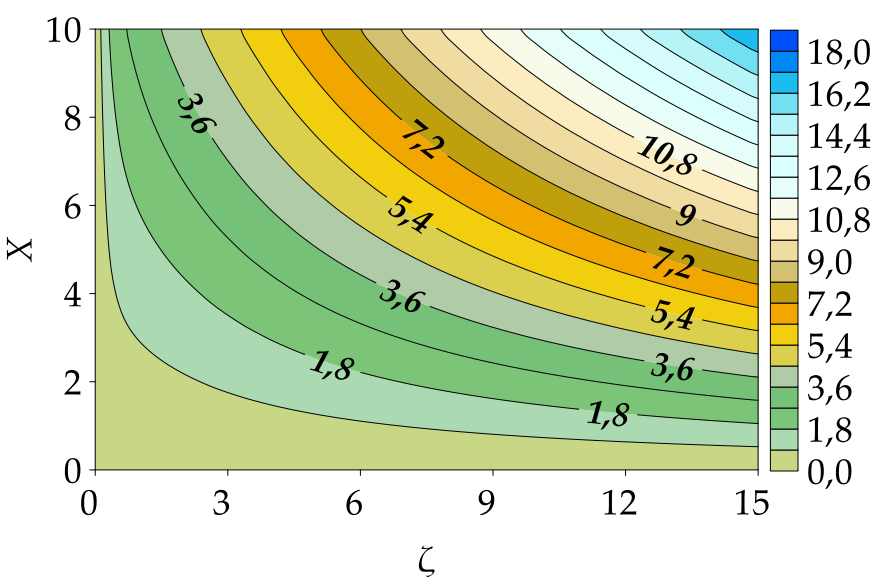

(C)

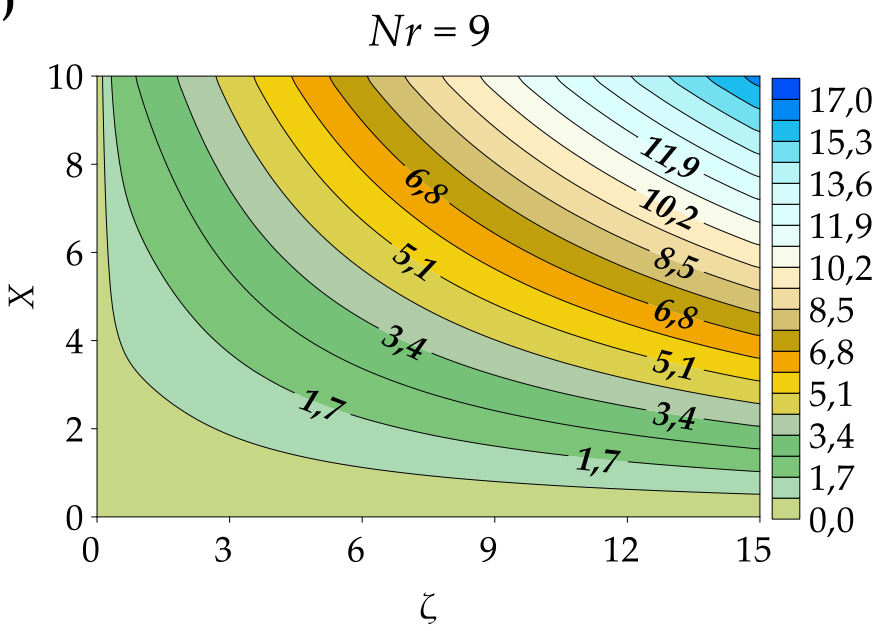




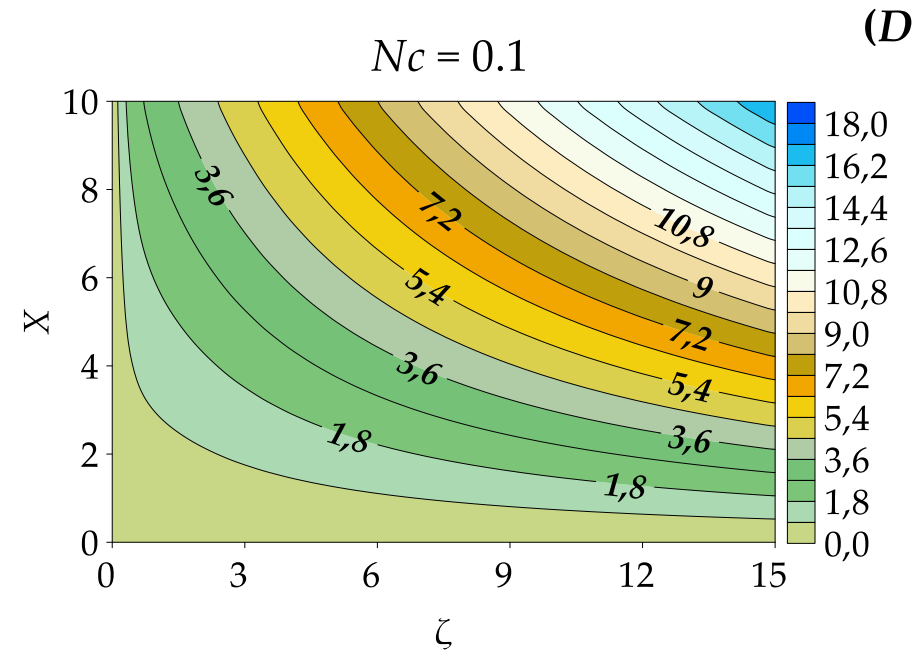

(D)

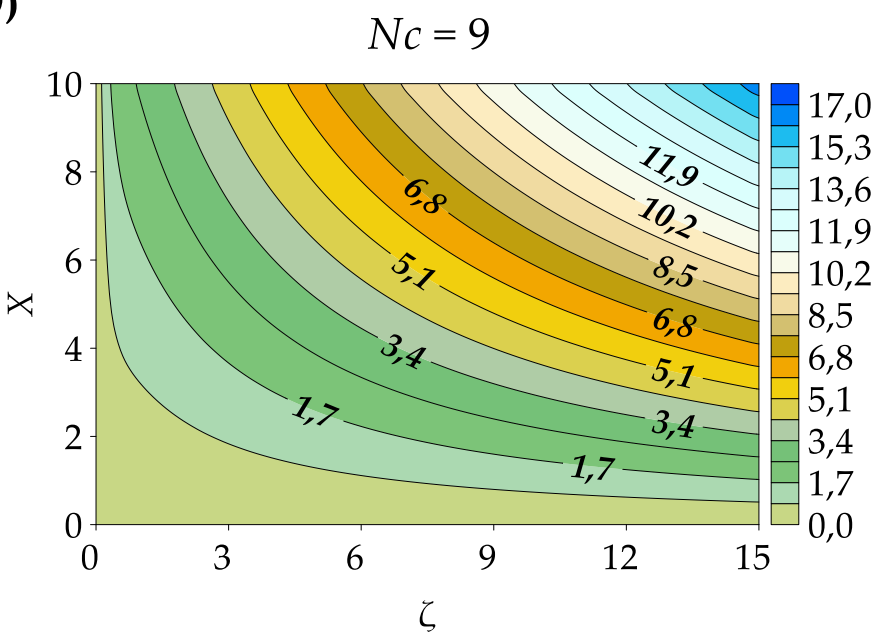

$(E)$
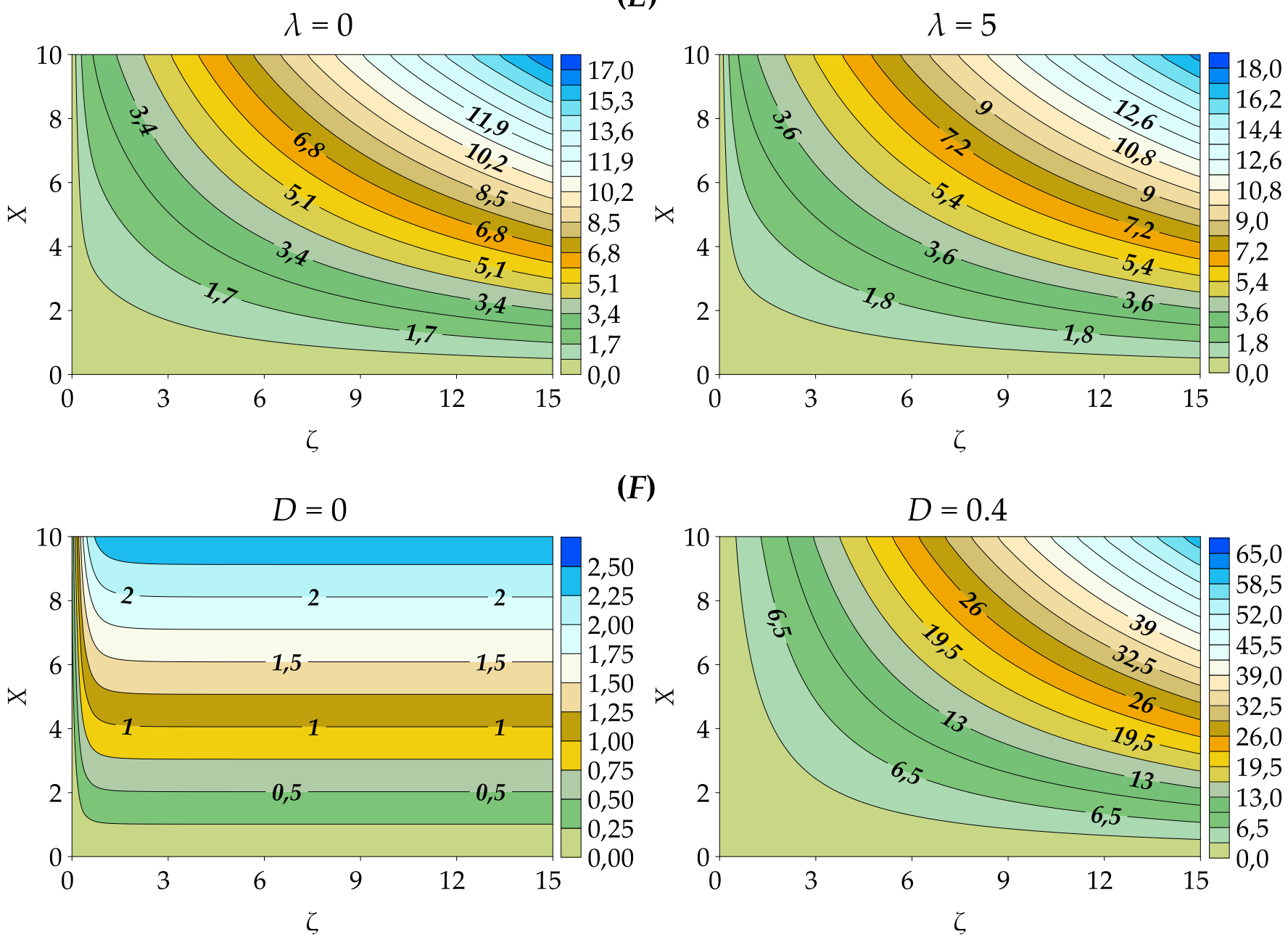

$(F)$

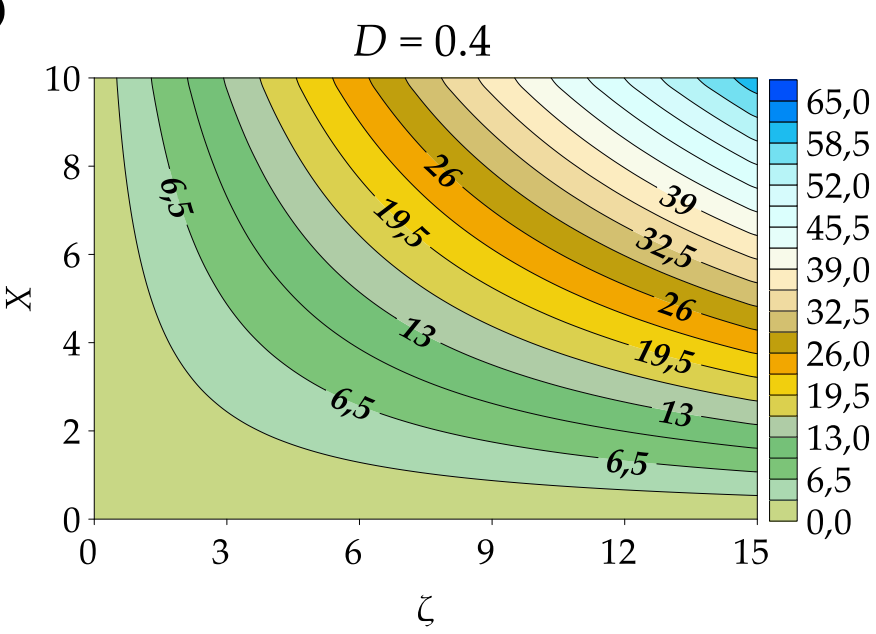

Fig.8: Two-dimensional shapes of streamlines for distinct values of $\alpha, M, N r, N c, \lambda$, and $D$. 


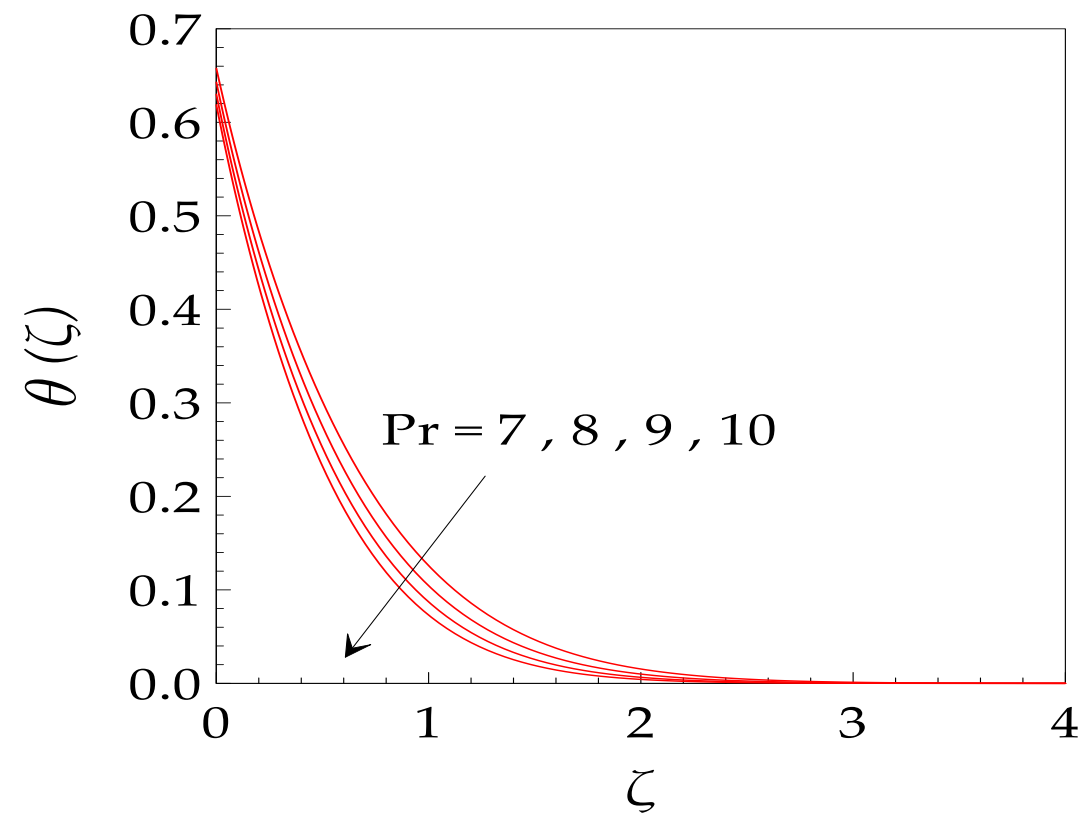

Fig.9: Profiles of $\theta(\zeta)$ at various values of $\operatorname{Pr}$

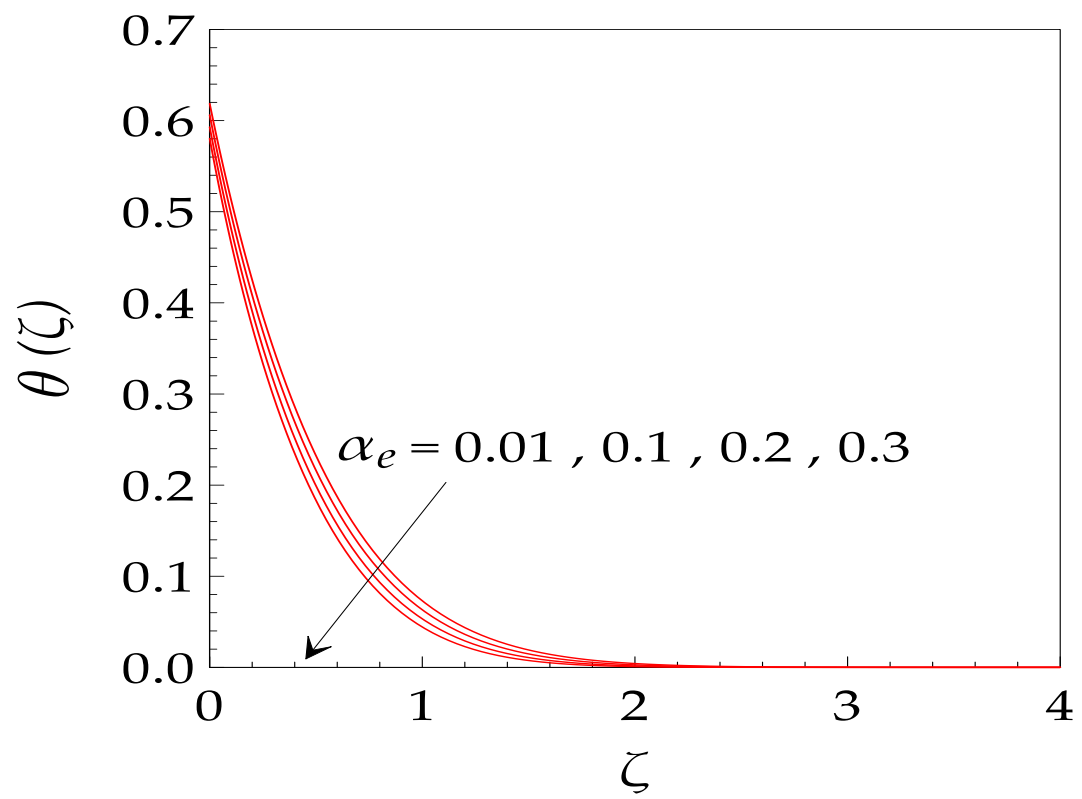

Fig.10: Profiles of $\theta(\zeta)$ at various values of $\alpha_{e}$. 


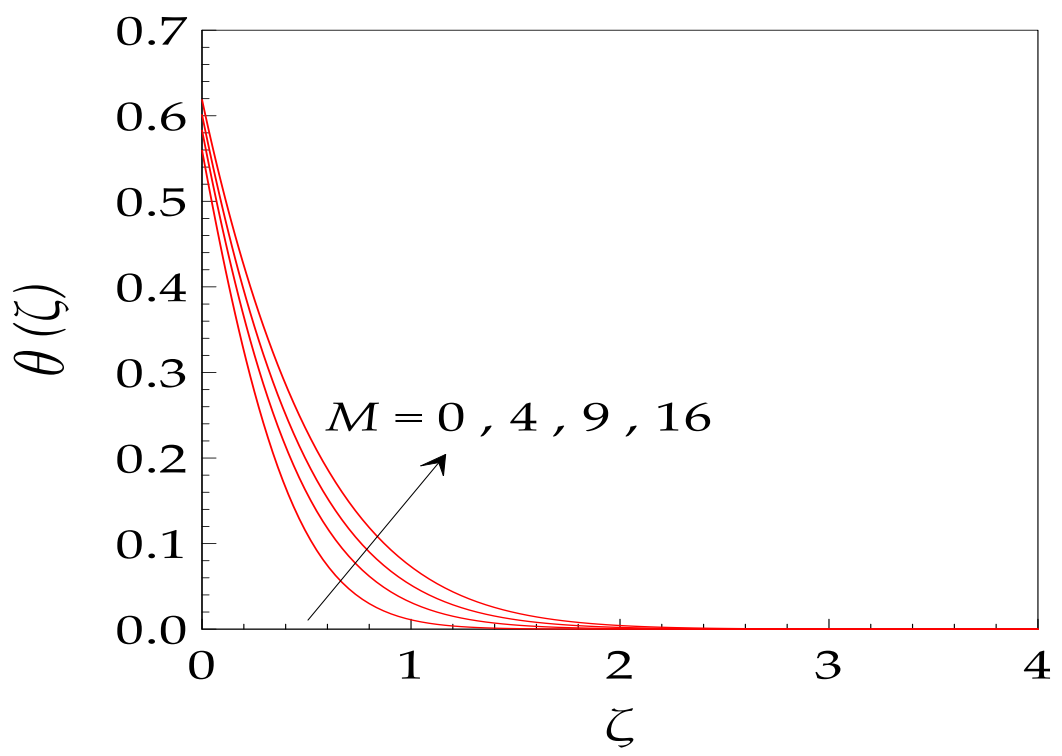

Fig.11: Profiles of $\theta(\zeta)$ at various values of $M$.

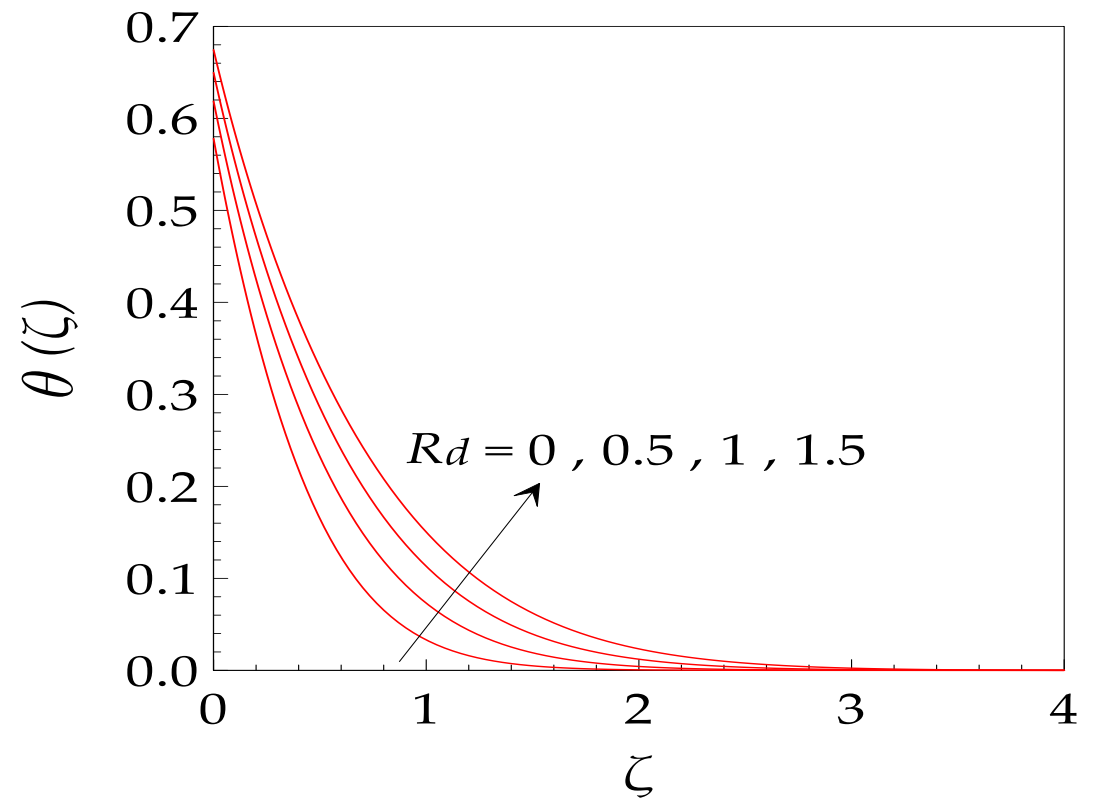

Fig.12: Profiles of $\theta(\zeta)$ at various values of $R d$ 


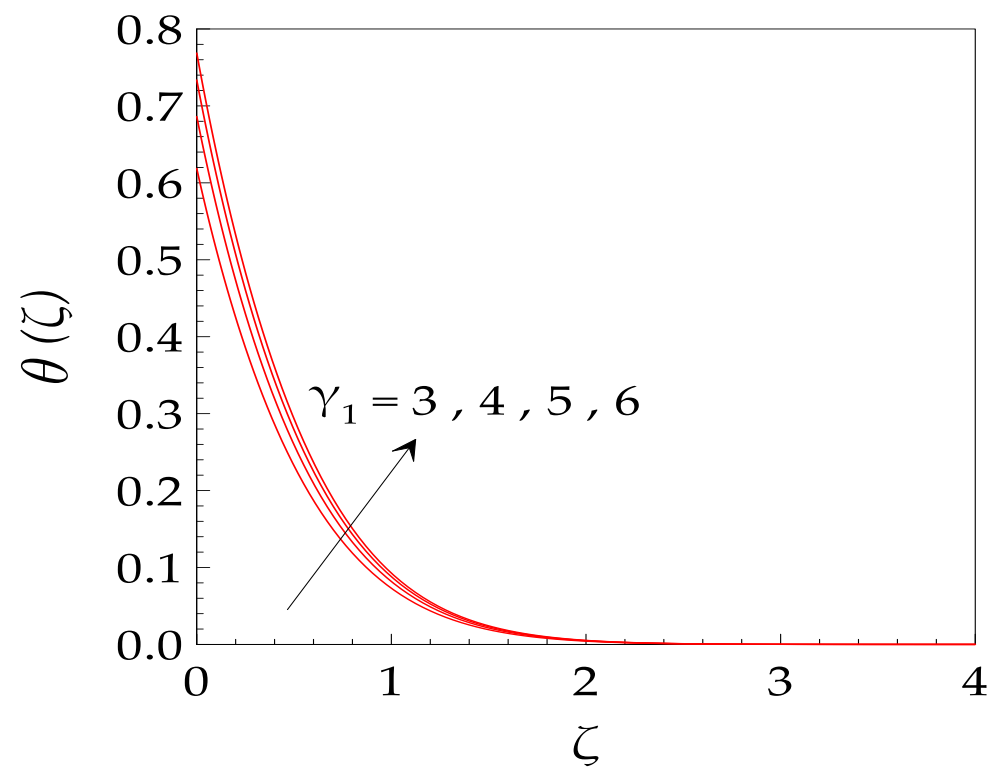

Fig.13: Profiles of $\theta(\zeta)$ at various values of $\gamma_{1}$

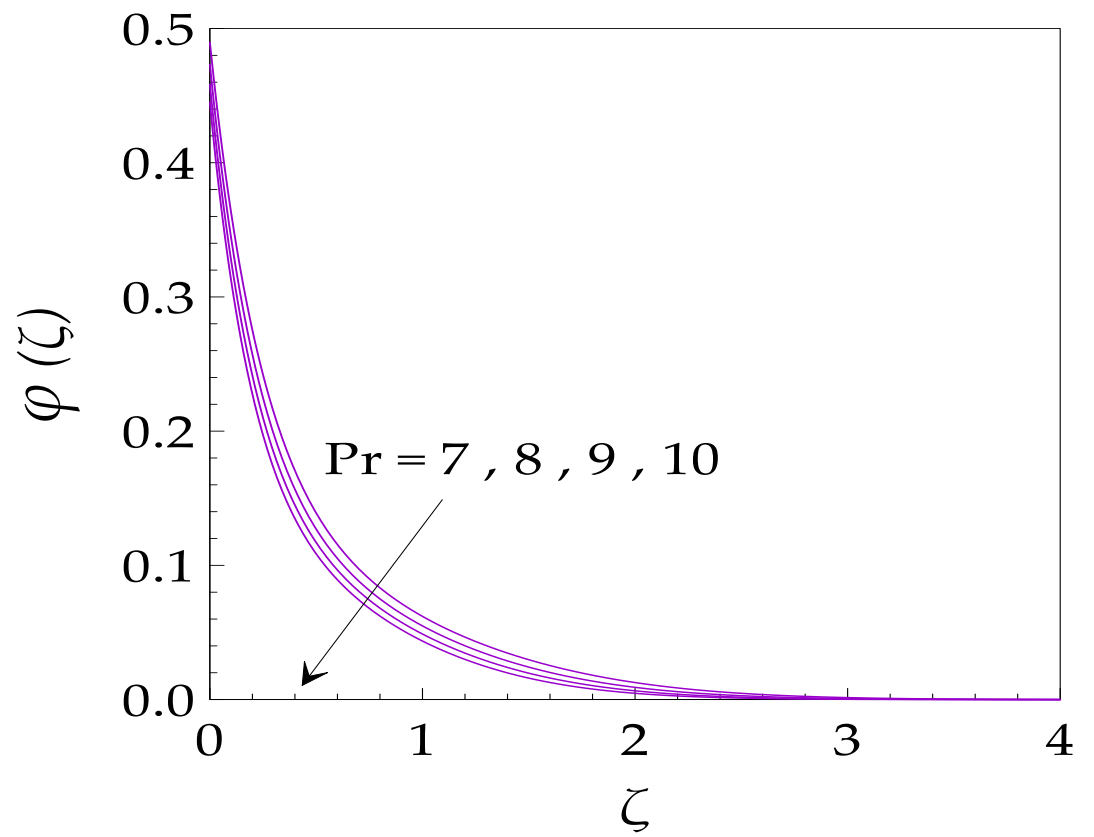

Fig.14: Profiles of $\phi(\zeta)$ at various values of $\operatorname{Pr}$ 


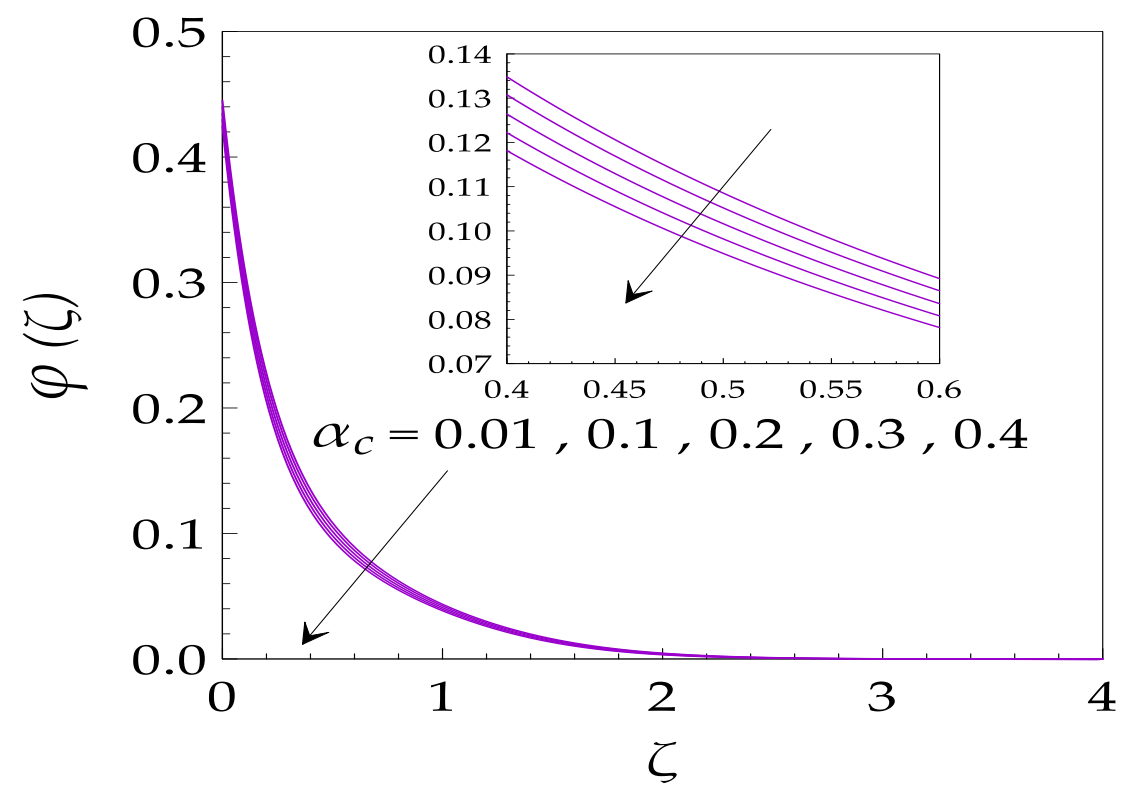

Fig.15: Profiles of $\phi(\zeta)$ at various values of $\alpha_{c}$

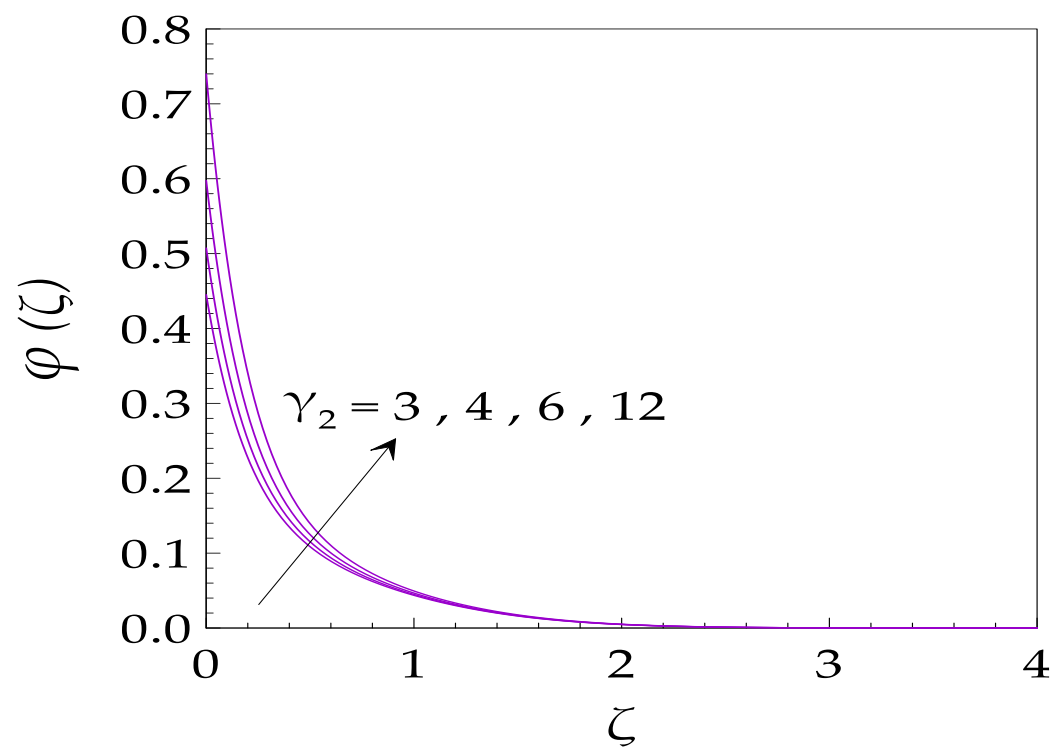

Fig.16: Profiles of $\phi(\zeta)$ at various values of $\gamma_{2}$ 


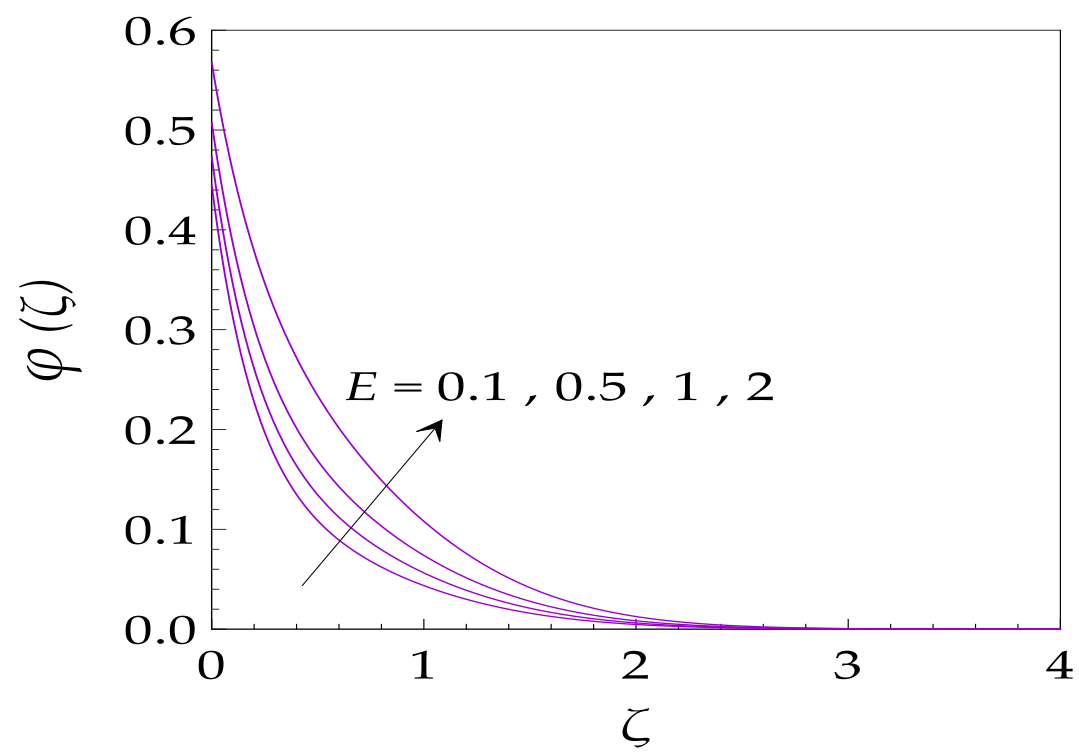

Fig.17: Profiles of $\phi(\zeta)$ at various values of $E$

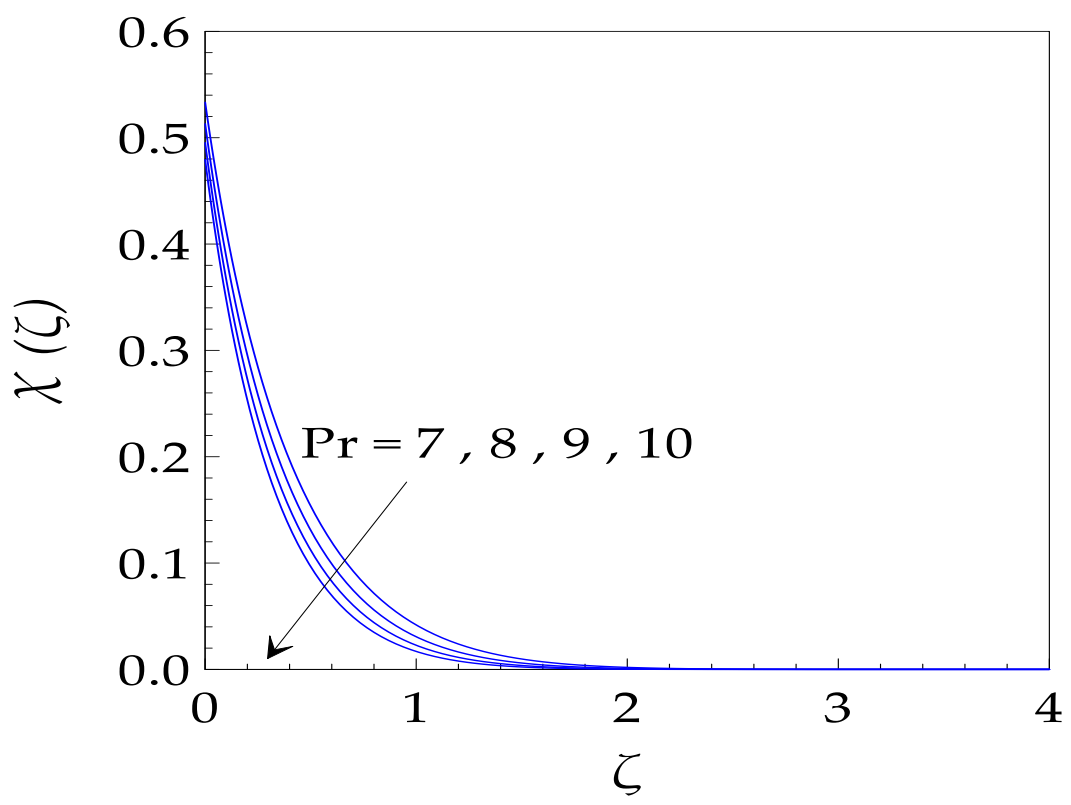

Fig.18: Profiles of $\chi(\zeta)$ at various values of $\operatorname{Pr}$ 


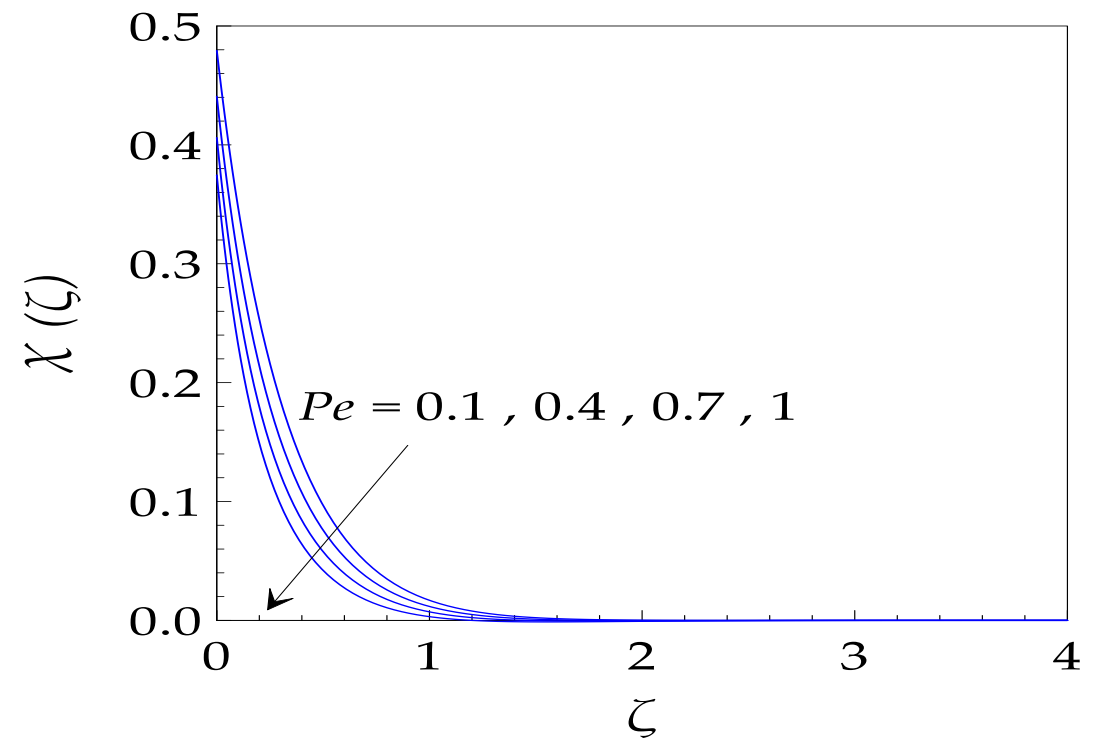

Fig.19: Profiles of $\chi(\zeta)$ at various values of $P e$

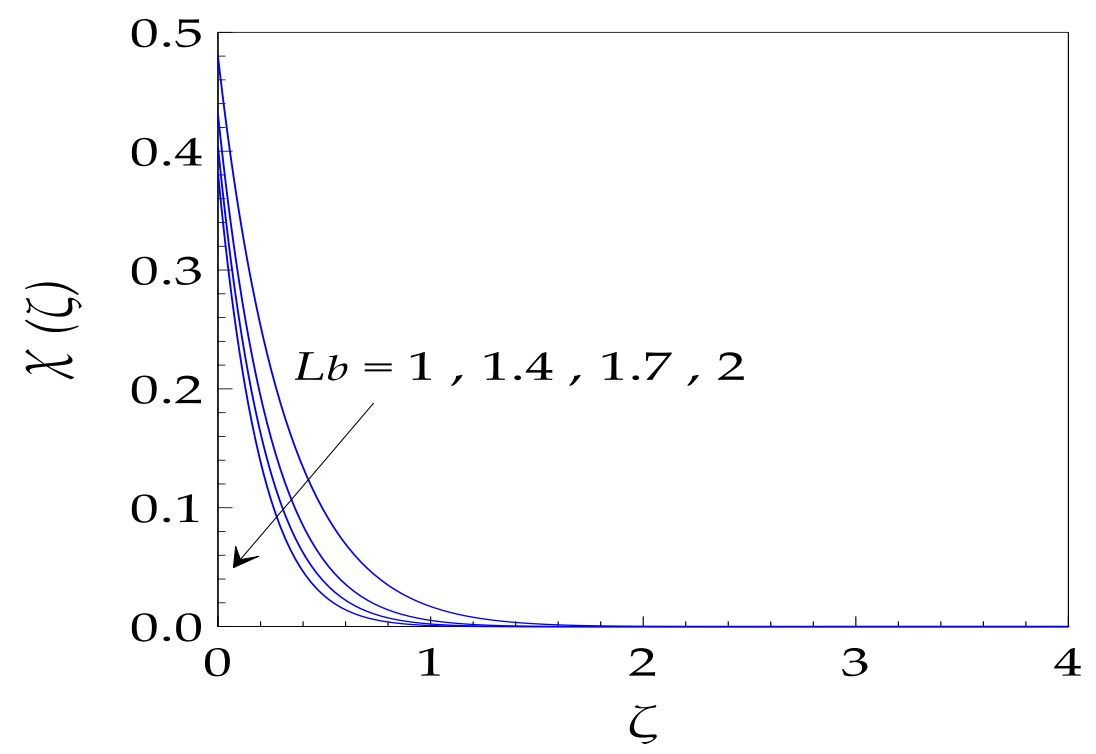

Fig.20: Profiles of $\chi(\zeta)$ at various values of $L b$ 


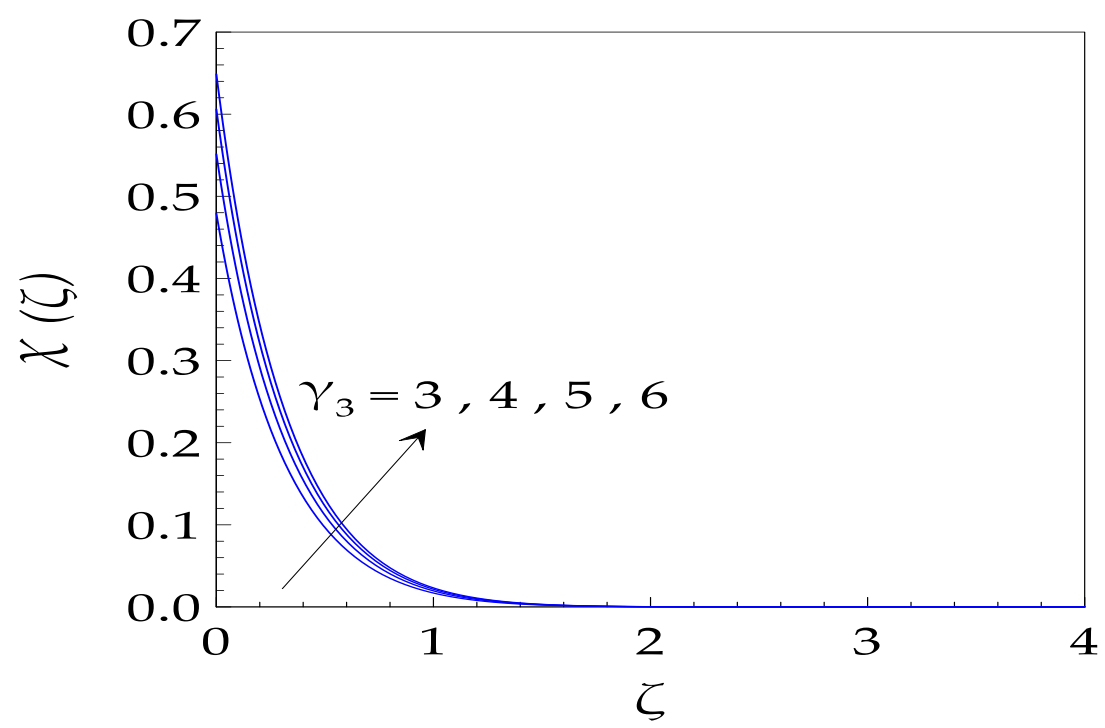

Fig.21: Profiles of $\chi(\zeta)$ at various values of $\gamma_{3}$

\subsection{Behaviors of the Engineering Quantities of interest:}

Starting from the SLLM numerical data findings, the practical values of the viscous friction amount $f^{\prime \prime}(0)$, the surface drag force coefficient $S_{f r}$, the dimensionless wall temperature $\theta(0)$, the reduced Nusselt number $N u_{r}$, the dimensionless wall nanoparticles concentration $\phi(0)$, the reduced Sherwood number $S h_{r}$, the dimensionless wall microorganisms concentration $\chi(0)$, and the reduced microorganisms transportation rate coefficient $\mathrm{Nm}_{r}$ are presented and evaluated fittingly in Table 5, Table 6, Table 7, and Table 8 via their rates of variation against the increasing values of the involved parameters $\alpha, M, N r, N c, \lambda, D, \operatorname{Pr}, \alpha_{e}$, $R d, \gamma_{1}, \alpha_{c}, \gamma_{2}, E, P e, L b$, and $\gamma_{3}$ by utilizing the SLRM statistical procedure. From Table 5, it is witnessed that the viscous frictional interaction between the nanofluid and the stretching sheet is weakened remarkably with the escalating values of the parameters $\alpha, \lambda$, and $D$, in which a maximum assessment in the reduction rate of about 10.62345197 is attained linearly in term of the surface drag force coefficient $S_{f r}$ (i. e., $\Delta S_{f r} / \Delta \alpha=-10.62345197$ ), when the non-Newtonian viscoelastic parameter $\alpha$ is augmented from 0 to 0.3 . However, a slight strengthening in the viscous frictional effect is noticed for the mounting estimates of the parameters $M, N r$, and $N c$. Thermally, it is perceived from Table 6 that the dimensionless wall temperature $\theta(0)$ improves gradually with the growing values of the parameter $M, R d$, and $\gamma_{1}$, while it declines a bit with the upsurging values of the parameters $\operatorname{Pr}$ and $\alpha_{e}$. Dissimilarly, an opposite trend is noted for the reduced Nusselt number $N u_{r}$ towards those parameres, except for the parameters $R d$ and $\gamma_{1}$, in which the dimensionless physical quantities $\theta(0)$ and $N u_{r}$ are progressed qualitatively in the same manner. An excellent enhancement in the thermal performance up to 0.782199006 is reached lineally for the engineering quantity $N u_{r}$ (i. e. , $\left.\Delta N u_{r} / \Delta R d=0.782199006\right)$ with the higher estimates of the thermal radiation parameter $(0 \leq R d \leq 1.5)$. In another context, the mass transport feature of the present nanofluid 
flow is depicted quantitatively in Table 7 via the rescaled wall nanoparticles concentration $\phi(0)$ and the reduced Sherwood number $S h_{r}$. From this tabular demonstration, it is comprehended that for the higher estimates of the parameters $\gamma_{2}$ and $E$, the solid nanoparticles concentrate more at the impermeable boundary, while a reverse tendency is seen for the parameters $\operatorname{Pr}$ and $\alpha_{c}$. Also, it is proved that the mass transfer rate becomes essential for larger values of the parameters $\operatorname{Pr}, \alpha_{c}, \gamma_{2}$, and decreases significantly with the elevating values of the parameter $E$. A notable enrichment in the mass transport process of approximately 0.156162827 and 0.153810650 is achieved linearly in term of the reduced Sherwood number $S h_{r}$ (i. e. , $\Delta S h_{r} / \Delta \alpha_{c}=0.156162827$ and $\left.\Delta S h_{r} / \Delta \gamma_{2}=0.153810650\right)$ with the improving values of the concentration relaxation parameter $\alpha_{c}\left(0.01 \leq \alpha_{c} \leq 0.4\right)$ and the nanoparticles concentration Biot number $\gamma_{2}\left(3 \leq \gamma_{2} \leq 12\right)$, correspondingly. On the other hand, Table 7 is established to reflect the behaviors of the dimensionless wall microorganisms concentration $\chi(0)$ and the reduced microorganisms transportation rate coefficient $N m_{x}$ versus the increasing values given to the control parameters $\mathrm{Pr}, \mathrm{Pe}, L b$, and $\gamma_{3}$. Among all of those embedded parameters, only the parameter $\gamma_{3}$ who has an increasing consequence on the concentration $\chi(0)$ of motile microorganisms at the wall, whereas all parameters exhibit a decreasing influence on the rate of motile microorganisms transportation $\mathrm{Nm}_{r}$. A distinguished augmentation in the reduced quantity $N m_{r}$ of around 0.349076951 is happened (i. e. , $\Delta N m_{r} / \Delta P e=0.349076951$ ), when the bioconvection Peclet number $P e$ is raised from 0.1 to 1 . Using the slope of linear regression through the data points used by Koriko et al. [57] it is worth concluding that with an increase in Prandtl number, Bioconvection Peclet number, and Bioconvection Lewis number, the concentration of motile microorganisms at the wall $\chi(0)$ decreases at the rate of $-0.017959017,-0.116358984$, and -0.098275597 respectively; see Table 8. But, the concentration of motile microorganisms at the wall is an increasing property of Microorganisms concentration Biot number.

Table 5: SLLM numerical estimations of $f^{\prime \prime}(0)$ and $S_{f r}$, when $N_{T}=70$ and $\zeta_{\infty}=15$.

\begin{tabular}{|c|c|c|c|}
\hline Parameters & Values & $-f^{\prime \prime}(0)$ & $C_{f r}$ \\
\hline \multirow{4}{*}{$\alpha$} & 0.0 & 3.638692654 & 3.638692654 \\
\hline & 0.1 & 3.866320694 & 2.706424486 \\
\hline & 0.2 & 4.140238186 & 1.656095274 \\
\hline & 0.3 & 4.476517345 & 0.447651734 \\
\hline \multicolumn{2}{|c|}{ Slope (linear regression) } & 2.787391565 & -10.62345197 \\
\hline \multirow{4}{*}{$M$} & 0 & 0.892137165 & 0.624496015 \\
\hline & 4 & 2.051706377 & 1.436194464 \\
\hline & 9 & 2.941115123 & 2.058780586 \\
\hline & 16 & 3.866320694 & 2.706424486 \\
\hline \multicolumn{2}{|c|}{ Slope (linear regression) } & 0.18102429 & 0.126717003 \\
\hline
\end{tabular}




\begin{tabular}{|c|c|c|c|}
\hline \multirow{4}{*}{$N r$} & 0.1 & 3.866320694 & 2.706424486 \\
\hline & 3.0 & 4.052369177 & 2.836658424 \\
\hline & 6.0 & 4.242697584 & 2.969888309 \\
\hline & 9.0 & 4.430679074 & 3.101475351 \\
\hline \multicolumn{2}{|c|}{ Slope (linear regression) } & 0.063411795 & 0.044388256 \\
\hline \multirow{4}{*}{$N c$} & 0.1 & 3.866320694 & 2.706424486 \\
\hline & 3.0 & 4.071468218 & 2.850027753 \\
\hline & 6.0 & 4.289917197 & 3.002942038 \\
\hline & 9.0 & 4.516378370 & 3.161464859 \\
\hline \multicolumn{2}{|c|}{ Slope (linear regression) } & 0.073025290 & 0.051117703 \\
\hline \multirow{6}{*}{$\lambda$} & 0 & 3.959995115 & 2.771996580 \\
\hline & 1 & 3.866320694 & 2.706424486 \\
\hline & 2 & 3.774033325 & 2.641823327 \\
\hline & 3 & 3.682993593 & 2.578095515 \\
\hline & 4 & 3.593085814 & 2.515160070 \\
\hline & 5 & 3.504212497 & 2.452948748 \\
\hline \multicolumn{2}{|c|}{ Slope (linear regression) } & -0.091133070 & -0.063793149 \\
\hline \multirow{5}{*}{$D$} & 0.0 & 4.245251861 & 2.971676303 \\
\hline & 0.1 & 3.866320694 & 2.706424486 \\
\hline & 0.2 & 3.474757097 & 2.432329968 \\
\hline & 0.3 & 3.072005527 & 2.150403869 \\
\hline & 0.4 & 2.659117037 & 1.861381926 \\
\hline \multicolumn{2}{|c|}{ Slope (linear regression) } & -3.966584815 & -2.776609371 \\
\hline
\end{tabular}

Table 6: SLLM numerical estimations of $\theta(0)$ and $N u_{r}$, when $N_{T}=70$ and $\zeta_{\infty}=15$.

\begin{tabular}{|c|c|c|c|}
\hline Parameter & Value & $\theta(0)$ & $N u_{r}$ \\
\hline \multirow{4}{*}{$\operatorname{Pr}$} & 7 & 0.657727447 & 1.540226484 \\
\hline & 8 & 0.642868926 & 1.607089829 \\
\hline & 9 & 0.629984286 & 1.665070710 \\
\hline & 10 & 0.618694475 & 1.715874860 \\
\hline \multicolumn{2}{|c|}{ Slope (linear regression) } & -0.012998356 & 0.058492601 \\
\hline \multirow{4}{*}{$\alpha_{e}$} & 0.01 & 0.618694475 & 1.715874860 \\
\hline & 0.10 & 0.605440048 & 1.775519782 \\
\hline & 0.20 & 0.592068698 & 1.835690858 \\
\hline & 0.30 & 0.579358343 & 1.892887454 \\
\hline \multicolumn{2}{|c|}{ Slope (linear regression) } & -0.135336090 & 0.609012408 \\
\hline \multirow{3}{*}{$M$} & 0 & 0.559084413 & 1.984120140 \\
\hline & 4 & 0.582937728 & 1.876780221 \\
\hline & 9 & 0.601065946 & 1.795203242 \\
\hline
\end{tabular}




\begin{tabular}{|c|c|c|c|}
\hline & 16 & 0.618694475 & 1.715874860 \\
\hline Slope (lir & ession) & 0.003625446 & -0.016314508 \\
\hline \multirow{4}{*}{$R d$} & 0.0 & 0.578159147 & 1.265522558 \\
\hline & 0.5 & 0.618694475 & 1.715874860 \\
\hline & 1.0 & 0.649771178 & 2.101372928 \\
\hline & 1.5 & 0.674574904 & 2.440688212 \\
\hline \multicolumn{2}{|c|}{ Slope (linear regression) } & 0.064064795 & 0.782199006 \\
\hline \multirow{4}{*}{$\gamma_{1}$} & 3 & 0.618694475 & 1.715874860 \\
\hline & 4 & 0.686258782 & 1.882447303 \\
\hline & 5 & 0.733694971 & 1.997287711 \\
\hline & 6 & 0.768766049 & 2.081105557 \\
\hline \multicolumn{2}{|c|}{ Slope (linear regression) } & 0.049765091 & 0.121053250 \\
\hline
\end{tabular}

Table 7: SLLM numerical estimations of $\phi(0)$ and $S h_{r}$, when $N_{T}=70$ and $\zeta_{\infty}=15$.

\begin{tabular}{|c|c|c|c|}
\hline Parameter & Value & $\phi(0)$ & $S h_{r}$ \\
\hline \multirow{4}{*}{$\operatorname{Pr}$} & 7 & 0.489659333 & 1.531021999 \\
\hline & 8 & 0.473157991 & 1.580526025 \\
\hline & 9 & 0.458419820 & 1.624740539 \\
\hline & 10 & 0.445101386 & 1.664695840 \\
\hline \multicolumn{2}{|c|}{ Slope (linear regression) } & -0.014841201 & 0.044523604 \\
\hline \multirow{5}{*}{$\alpha_{c}$} & 0.01 & 0.445101386 & 1.664695840 \\
\hline & 0.10 & 0.440237352 & 1.679287943 \\
\hline & 0.20 & 0.434953197 & 1.695140407 \\
\hline & 0.30 & 0.429806192 & 1.710581423 \\
\hline & 0.40 & 0.424804044 & 1.725587865 \\
\hline \multicolumn{2}{|c|}{ Slope (linear regression) } & -0.052054276 & 0.156162827 \\
\hline \multirow{4}{*}{$\gamma_{2}$} & 3 & 0.445101386 & 1.664695840 \\
\hline & 4 & 0.507402887 & 1.970388450 \\
\hline & 6 & 0.597552330 & 2.414686017 \\
\hline & 12 & 0.739919185 & 3.120969774 \\
\hline \multicolumn{2}{|c|}{ Slope (linear regression) } & 0.031116128 & 0.153810650 \\
\hline \multirow{4}{*}{$E$} & 0.1 & 0.445101386 & 1.664695840 \\
\hline & 0.5 & 0.473447418 & 1.579657743 \\
\hline & 1.0 & 0.507717464 & 1.476847605 \\
\hline & 2.0 & 0.567783605 & 1.296649184 \\
\hline Slope (linear & ssion) & 0.064293879 & -0.192881637 \\
\hline
\end{tabular}

Table 8: SLLM numerical estimations of $\chi(0)$ and $N m_{r}$, when $N_{T}=70$ and $\zeta_{\infty}=15$.

\begin{tabular}{llll}
\hline Parameter & Value & $\chi(0)$ & $N m_{r}$ \\
\hline & &
\end{tabular}




\begin{tabular}{|c|c|c|c|}
\hline \multirow{4}{*}{$\operatorname{Pr}$} & 7 & 0.533303690 & 1.400088928 \\
\hline & 8 & 0.513042333 & 1.460872998 \\
\hline & 9 & 0.495226134 & 1.514321596 \\
\hline & 10 & 0.479379032 & 1.561862902 \\
\hline \multicolumn{2}{|c|}{ Slope (linear regression) } & -0.017959017 & $\mathbf{0 . 0 5 3 8 7 7 0 5 2}$ \\
\hline \multirow{4}{*}{$P e$} & 0.1 & 0.479379032 & 1.561862902 \\
\hline & 0.4 & 0.440811083 & 1.677566751 \\
\hline & 0.7 & 0.406059216 & 1.781822351 \\
\hline & 1.0 & 0.374604004 & 1.876187986 \\
\hline \multicolumn{2}{|c|}{ Slope (linear regression) } & -0.116358984 & 0.349076951 \\
\hline \multirow{4}{*}{$L b$} & 1.0 & 0.479379032 & 1.561862902 \\
\hline & 1.4 & 0.430667714 & 1.707996857 \\
\hline & 1.7 & 0.403429363 & 1.789711910 \\
\hline & 2.0 & 0.381266164 & 1.856201505 \\
\hline \multicolumn{2}{|c|}{ Slope (linear regression) } & -0.098275597 & 0.294826792 \\
\hline \multirow{4}{*}{$\gamma_{3}$} & 3 & 0.479379032 & 1.561862902 \\
\hline & 4 & 0.551596515 & 1.793613939 \\
\hline & 5 & 0.606222177 & 1.968889111 \\
\hline & 6 & 0.648985330 & 2.106088015 \\
\hline \multicolumn{2}{|c|}{ Slope (linear regression) } & 0.056344456 & 0.180795051 \\
\hline
\end{tabular}

\section{Concluding Remarks}

In this survey, the crucial characteristics of MHD mixed bioconvective phenomenon that can take place within the radiative-reactive flow of a non-Newtonian nanofluid over a vertical stretching sheet have been investigated comprehensively by adopting the Walters-B viscoelastic rheological model and introducing the concept of non-Fourier's double diffusion theory along with the Brownian and thermophoresis aspects. Further, the impact of Arrhenius activation energy has been scrutinized properly through the reactive sink term incorporated in the concentration equation. By using the spectral local linearization method (SLLM), the resulting nonlinear differential system has been tackled numerically for a set of Dirichlet and Robin boundary conditions to reveal the exciting features of the present boundary layer flow. Based on the results of various illustrations, the following main conclusions can be itemized as follows:

- The SLLM numerical method provides robustness results with a higher level of exactness and low-computational cost.

- After performing a successful validation of the proposed SLLM numerical algorithm, the resulting datasets are presented in tables and graphs with an absolute accuracy of the order of $10^{-10}$. 
- The nanofluid velocity profile $f^{\prime}(\zeta)$ exhibits a declining behavior with the magnifying values of the parameters $\alpha, M, N r$, and $N c$,while an opposite tendency is depicted for the parameters $\lambda$ and $D$.

- A noticeable decline in the nanofluid temperature distribution $\theta(\zeta)$ is noted for the higher estimates of the parameters $\operatorname{Pr}$ and $\alpha_{e}$, whereas a significant boost in the nanofluid temperature is remarked with the strengthening values of the parameters $M, R d$, and $\gamma_{1}$.

- The nanoparticles concentration distribution $\phi(\zeta)$ can be heightened considerably either by diminishing the values of the parameters $\operatorname{Pr}$ and $\alpha_{c}$ or by increasing the values of the parameters $\gamma_{2}$ and $E$.

- The motile microorganisms concentration profile $\chi(\zeta)$ shows a favorable decay with the rise in the values of the parameters $\operatorname{Pr}, P e$, and $L b$, however a reverse trend is noticed for the parameter $\gamma_{3}$.

- An attractive reduction in the surface drag force coefficient $S_{f r}$ is achievable via the intensifying values of the non-Newtonian parameter $\alpha$.

- A higher enhancement in the reduced engineering quantities $N u_{r}, S h_{r}$, and $N m_{r}$ is observed respectively with the growing values of the parameters $R d, \alpha_{c}$, and $P e$.

- The concentration of motile microorganisms at the wall $\chi(0)$ is a decreasing property of Prandtl number, bioconvection Peclet number, and bioconvection Lewis number, but an increasing function of microorganisms concentration Biot number.

\section{Declaration of competing interest}

The authors affirm that they have no known competing financial interests or personal relationships that could have influenced the scientific work reported in this paper.

\section{Author contributions}

Conceptualization, AW; Methodology, ILA; Validation, UK; Formal analysis, AMA; Writing-original draft preparation, AW, ILA, UK, and AMA; Writing-review and editing, AW, ILA, UK, and AMA Revision \$\$

\section{References}

[1] S.U.S. Choi, J.A. Eastman, Enhancing conductivity of fluids with nanoparticles, Developments and Applications of Non-Newtonian Flows, D. A. Siginer H. P. Wang, Eds., Am. Soc. Mech. Eng. New York. 66 (1995) 99-105.

[2] J. Buongiorno, Convective Transport in Nanofluids, J. Heat Transfer. 128 (2006) 240-250. 
doi:10.1115/1.2150834.

[3] W.A. Khan, I. Pop, Boundary-layer flow of a nanofluid past a stretching sheet, Int. J. Heat Mass Transf. 53 (2010) 2477-2483. doi:10.1016/j.ijheatmasstransfer.2010.01.032.

[4] M. Zaydan, A. Wakif, I.L. Animasaun, U. Khan, D. Baleanu, R. Sehaqui, Significances of Blowing and Suction Processes on the Occurrence of Thermo-Magneto-Convection Phenomenon in a Narrow Nanofluidic Medium: A Revised Buongiorno's Nanofluid Model, Case Stud. Therm. Eng. (2020) 100726. doi:10.1016/j.csite.2020.100726.

[5] M. Sheikholeslami, M.M. Bhatti, Forced convection of nanofluid in presence of constant magnetic field considering shape effects of nanoparticles, Int. J. Heat Mass Transf. 111 (2017) 1039-1049. doi:https://doi.org/10.1016/j.ijheatmasstransfer.2017.04.070.

[6] M. Turkyilmazoglu, Condensation of laminar film over curved vertical walls using single and twophase nanofluid models, Eur. J. Mech. - B/Fluids. 65 (2017) 184-191. doi:https://doi.org/10.1016/j.euromechflu.2017.04.007.

[7] M.M. Bhatti, M.M. Rashidi, Effects of thermo-diffusion and thermal radiation on Williamson nanofluid over a porous shrinking/stretching sheet, J. Mol. Liq. 221 (2016) 567-573. doi:https://doi.org/10.1016/j.molliq.2016.05.049.

[8] T. Hayat, I. Ullah, M. Waqas, A. Alsaedi, MHD stratified nanofluid flow by slandering surface, Phys. Scr. 93 (2018) 115701. doi:10.1088/1402-4896/aae1a2.

[9] T. Hayat, M. Ijaz, S. Qayyum, M. Ayub, A. Alsaedi, Mixed convective stagnation point flow of nanofluid with Darcy-Fochheimer relation and partial slip, Results Phys. 9 (2018) 771-778. doi:https://doi.org/10.1016/j.rinp.2018.02.073.

[10] K.L. Hsiao, Micropolar nanofluid flow with MHD and viscous dissipation effects towards a stretching sheet with multimedia feature, Int. J. Heat Mass Transf. 112 (2017) 983-990. doi:10.1016/j.ijheatmasstransfer.2017.05.042.

[11] B.J. Gireesha, B. Mahanthesh, G.T. Thammanna, P.B. Sampathkumar, Hall effects on dusty nanofluid two-phase transient flow past a stretching sheet using KVL model, J. Mol. Liq. 256 (2018) 139-147. doi:https://doi.org/10.1016/j.molliq.2018.01.186.

[12] K. Anantha Kumar, J. V Ramana Reddy, V. Sugunamma, N. Sandeep, Magnetohydrodynamic Cattaneo-Christov flow past a cone and a wedge with variable heat source/sink, Alexandria Eng. J. 57 (2018) 435-443. doi:10.1016/j.aej.2016.11.013.

[13] K. Anantha Kumar, V. Sugunamma, N. Sandeep, Physical aspects on unsteady MHD-free convective stagnation point flow of micropolar fluid over a stretching surface, Heat Transf. Res. 48 (2019) 3968-3985. doi:10.1002/htj.21577.

[14] K.A. Kumar, V. Sugunamma, N. Sandeep, M.T. Mustafa, Simultaneous solutions for first order and 
second order slips on micropolar fluid flow across a convective surface in the presence of Lorentz force and variable heat source/sink, Sci. Rep. 9 (2019) 14706. doi:10.1038/s41598-019-51242-5.

[15] K.A. Kumar, V. Sugunamma, N. Sandeep, S. Sivaiah, Physical Aspects on MHD Micropolar Fluid Flow Past an Exponentially Stretching Curved Surface, Defect Diffus. Forum. 401 (2020) 79-91. doi:10.4028/www.scientific.net/DDF.401.79.

[16] K.A. Kumar, V. Sugunamma, N. Sandeep, Thermophoresis and Brownian motion effects on MHD micropolar nanofluid flow past a stretching surface with non-uniform heat source/sink, Comput. Therm. Sci. An Int. J. 12 (2020) 55-77. doi:10.1615/ComputThermalScien.2020027016.

[17] K.A. Kumar, V. Sugunamma, N. Sandeep, A non-Fourier heat flux model for magnetohydrodynamic micropolar liquid flow across a coagulated sheet, Heat Transf. Res. 48 (2019) 2819-2843. doi:10.1002/htj.21518.

[18] K. Anantha Kumar, V. Sugunamma, N. Sandeep, Effect of thermal radiation on MHD Casson fluid flow over an exponentially stretching curved sheet, J. Therm. Anal. Calorim. 140 (2020) 2377-2385. doi:10.1007/s10973-019-08977-0.

[19] K. Walters, Second-order Effects in Elasticity, Plasticity and Fluid Dynamics, in: International Symposium, Haifa, Israel, April 23-27, 1962, 1964: pp. 507-519.

[20] D.W. Beard, K. Walters, Elastico-viscous boundary-layer flows I. Two-dimensional flow near a stagnation point, Math. Proc. Cambridge Philos. Soc. 60 (1964) 667-674. doi:10.1017/S0305004100038147.

[21] G.S. Seth, M.K. Mishra, R. Tripathi, MHD free convective heat transfer in a Walter's liquid-B fluid past a convectively heated stretching sheet with partial wall slip, J. Brazilian Soc. Mech. Sci. Eng. 40 (2018) 103. doi:10.1007/s40430-018-1028-5.

[22] M. Ayub, M.Y. Malik, M. Ijaz, M.S. Alqarni, A.S. Alqahtani, Cattaneo-Christov double-diffusion model for viscoelastic nanofluid with activation energy and nonlinear thermal radiation, Multidiscip. Model. Mater. Struct. 16 (2019) 93-120. doi:10.1108/MMMS-03-2019-0046.

[23] N.A. Hill, T.J. Pedley, Bioconvection, Fluid Dyn. Res. 37 (2005) 1-20. doi:10.1016/j.fluiddyn.2005.03.002.

[24] A. V Kuznetsov, A.A. Avramenko, Effect of small particles on this stability of bioconvection in a suspension of gyrotactic microorganisms in a layer of finite depth, Int. Commun. Heat Mass Transf. 31 (2004) 1-10. doi:https://doi.org/10.1016/S0735-1933(03)00196-9.

[25] A. V Kuznetsov, Nanofluid bioconvection in water-based suspensions containing nanoparticles and oxytactic microorganisms: oscillatory instability, Nanoscale Res. Lett. 6 (2011) 100. doi:10.1186/1556-276X-6-100.

[26] M.K. Nayak, J. Prakash, D. Tripathi, V.S. Pandey, S. Shaw, O.D. Makinde, 3D Bioconvective 
multiple slip flow of chemically reactive Casson nanofluid with gyrotactic micro-organisms, Heat Transf. Res. 49 (2020) 135-153. doi:10.1002/htj.21603.

[27] K.A. Kumar, V. Sugunamma, N. Sandeep, J.V.R. Reddy, Impact of Brownian motion and thermophoresis on bioconvective flow of nanoliquids past a variable thickness surface with slip effects, Multidiscip. Model. Mater. Struct. 15 (2019) 103-132. doi:10.1108/MMMS-02-2018-0023.

[28] O. A. Beg, MD. N. Kabir, MD. J. Uddin, A. I. MD. Ismail, Y. M. Alginahi, Numerical investigation of Von Karman swirling bioconvective nanofluid transport from a rotating disk in a porous medium with Stefan blowing and anisotropic slip effects, Proc IMechE Part C: J Mechanical Engineering Science, 0(0) 1-19, (2020).

[29] K. Al-Khaled, S.U. Khan, I. Khan, Chemically reactive bioconvection flow of tangent hyperbolic nanoliquid with gyrotactic microorganisms and nonlinear thermal radiation, Heliyon. 6 (2020) e03117. doi:10.1016/j.heliyon.2019.e03117.

[30] N. Shukla, P. Rana, S. Kuharat, O. A. Beg, Non-Similar Radiative Bioconvection Nanofluid Flow under Oblique Magnetic Field with Entropy Generation, Articles in Press, (2020). doi:10.22055/jacm.2020.33580.2250.

[31] K. Mohamed, T. Ismail, N. Nourreddine, S. Mohamed Rafik, Analytical study of nano-bioconvective flow in a horizontal channel using Adomian decomposition method, J. Comput. Appl. Res. Mech. Eng. 9 (2020) 245-258. doi:10.22061/jcarme.2018.3269.1367.

[32] R. Naz, M. Noor, T. Hayat, M. Javed, A. Alsaedi, Dynamism of magnetohydrodynamic cross nanofluid with particulars of entropy generation and gyrotactic motile microorganisms, Int. $\begin{array}{llllll}\text { Commun. } & \text { Heat } & \text { Mass } & \text { Transf. } & 110 & \text { (2020) }\end{array}$ doi:https://doi.org/10.1016/j.icheatmasstransfer.2019.104431.

[33] R. Naz, S. Tariq, H. Alsulami, Inquiry of entropy generation in stratified Walters' B nanofluid with swimming gyrotactic microorganisms, Alexandria Eng. J. 59 (2020) 247-261. doi:https://doi.org/10.1016/j.aej.2019.12.037.

[34] I. Tlili, H. Waqas, A. Almaneea, S.U. Khan, M. Imran, Activation Energy and Second Order Slip in Bioconvection of Oldroyd-B Nanofluid over a Stretching Cylinder: A Proposed Mathematical Model, Processes. 7 (2019) 914. doi:https://doi.org/10.3390/pr7120914.

[35] T. Abbas, T. Hayat, M. Ayub, M.M. Bhatti, A. Alsaedi, Electromagnetohydrodynamic nanofluid flow past a porous Riga plate containing gyrotactic microorganism, Neural Comput. Appl. 31 (2019) 1905-1913. doi:10.1007/s00521-017-3165-7.

[36] M.M. Bhatti, S.R. Mishra, T. Abbas, M.M. Rashidi, A mathematical model of MHD nanofluid flow having gyrotactic microorganisms with thermal radiation and chemical reaction effects, Neural Comput. Appl. 30 (2018) 1237-1249. doi:10.1007/s00521-016-2768-8. 
[37] A. Shahid, H. Huang, M.M. Bhatti, L. Zhang, R. Ellahi, Numerical Investigation on the Swimming of Gyrotactic Microorganisms in Nanofluids through Porous Medium over a Stretched Surface, Mathematics. 8 (2020) 380. doi:https://doi.org/10.3390/math8030380.

[38] C.S. Balla, C. Haritha, K. Naikoti, A.M. Rashad, Bioconvection in nanofluid-saturated porous square cavity containing oxytactic microorganisms, Int. J. Numer. Methods Heat \&amp; Fluid Flow. 29 (2019) 1448-1465. doi:10.1108/HFF-05-2018-0238.

[39] W.A. Khan, A.M. Rashad, M.M.M. Abdou, I. Tlili, Natural bioconvection flow of a nanofluid containing gyrotactic microorganisms about a truncated cone, Eur. J. Mech. - B/Fluids. 75 (2019) 133-142. doi:https://doi.org/10.1016/j.euromechflu.2019.01.002.

[40] A.M. Rashad, H.A. Nabwey, Gyrotactic mixed bioconvection flow of a nanofluid past a circular cylinder with convective boundary condition, J. Taiwan Inst. Chem. Eng. 99 (2019) 9-17. doi:https://doi.org/10.1016/j.jtice.2019.02.035.

[41] M. Sohail, R. Naz, S.I. Abdelsalam, On the onset of entropy generation for a nanofluid with thermal radiation and gyrotactic microorganisms through 3D flows, Phys. Scr. 95 (2020) 45206. doi:10.1088/1402-4896/ab3c3f.

[42] S.S. Motsa, A new spectral local linearization method for nonlinear boundary layer flow problems, J. Appl. Math. 2013 (2013) Article ID 423628, 15 pages. doi:10.1155/2013/423628.

[43] A. Wakif, Z. Boulahia, R. Sehaqui, Numerical analysis of the onset of longitudinal convective rolls in a porous medium saturated by an electrically conducting nanofluid in the presence of an external magnetic field, Results Phys. 7 (2017) 2134-2152. doi:10.1016/j.rinp.2017.06.003.

[44] A. Wakif, Z. Boulahia, A. Amine, I.L. Animasaun, M.I. Afridi, M. Qasim, R. Sehaqui, Magnetoconvection of alumina - water nanofluid within thin horizontal layers using the revised generalized Buongiorno's model, Front. Heat Mass Transf. 12 (2019) 1-15. doi:10.5098/hmt.12.3.

[45] A. Wakif, Z. Boulahia, F. Ali, M.R. Eid, R. Sehaqui, Numerical Analysis of the Unsteady Natural Convection MHD Couette Nanofluid Flow in the Presence of Thermal Radiation Using Single and Two-Phase Nanofluid Models for Cu-Water Nanofluids, Int. J. Appl. Comput. Math. 4, 81 (2018). doi:10.1007/s40819-018-0513-y.

[46] A. Wakif, M. Qasim, M.I. Afridi, S. Saleem, M.M. Al-Qarni, Numerical Examination of the Entropic Energy Harvesting in a Magnetohydrodynamic Dissipative Flow of Stokes' Second Problem: Utilization of the Gear-Generalized Differential Quadrature Method, J. Non-Equilibrium Thermodyn. (2019) 1-19. doi:10.1515/jnet-2018-0099.

[47] T. Thumma, A. Wakif, I.L. Animasaun, Generalized differential quadrature analysis of unsteady three-dimensional MHD radiating dissipative Casson fluid conveying tiny particles, Heat Transf. 49 (2020) 2595 - 2626. doi:10.1002/htj.21736. 
[48] G. Rasool, A. Wakif, Numerical Spectral Examination of EMHD Mixed Convective Flow of Second Grade Nanofluid towards a Vertical Riga Plate Using an Advanced Version of the Revised Buongiorno’s Nanofluid Model, J. Therm. Anal. Calorim. (2020). doi:10.1007/s10973-020-098658.

[49] A. Wakif, A novel numerical procedure for simulating steady MHD convective flows of radiative Casson fluids over a horizontal stretching sheet with irregular geometry under the combined influence of temperature-dependent viscosity and thermal conductivity, Math. Probl. Eng. 2020 (2020), Article ID 1675350, 20 pages. doi:10.1155/2020/1675350.

[50] H.I. Andersson, MHD flow of a viscoelastic fluid past a stretching surface, Acta Mech. 95 (1992) 227-230. doi:10.1007/BF01170814.

[51] S.A. M. Mehryan, F. Moradi Kashkooli, M. Soltani, K. Raahemifar, Fluid flow and heat transfer analysis of a nanofluid containing motile gyrotactic micro-organisms passing a nonlinear stretching vertical sheet in the presence of a non-uniform magnetic field; numerical approach, PLoS One. 11 (2016) e0157598. doi:10.1371/journal.pone.0157598.

[52] I. Wubshet, G. Gosa, Finite Element Method Solution of Boundary Layer Flow of Powell-Eyring Nanofluid over a Nonlinear Stretching Surface, J. Appl. Math. 2019 (2019) Article ID 3472518, 16 pages. doi:10.1155/2019/3472518.

[53] I.L. Animasaun, R.O. Ibraheem, B. Mahanthesh, H.A. Babatunde, A meta-analysis on the effects of haphazard motion of tiny/nano-sized particles on the dynamics and other physical properties of some fluids, Chinese J. Phys. 60 (2019) 676-687. doi:10.1016/j.cjph.2019.06.007.

[54] A. Wakif, I.L. Animasaun, P. V. Satya Narayana, G. Sarojamma, Meta-analysis on thermo-migration of tiny/nano-sized particles in the motion of various fluids, Chinese J. Phys. 68 (2020) 293-307. doi:10.1016/j.cjph.2019.12.002.

[55] A. Wakif, A. Chamkha, T. Thumma, I.L. Animasaun, R. Sehaqui, Thermal radiation and surface roughness effects on the thermo-magneto-hydrodynamic stability of alumina-copper oxide hybrid nanofluids utilizing the generalized Buongiorno's nanofluid model, J. Therm. Anal. Calorim. 143 (2021) 1201-1220. doi:10.1007/s10973-020-09488-z.

[56] M. Ghalambaz, E. Izadpanahi, A. Noghrehabadi, A. Chamkha, Study of the boundary layer heat transfer of nanofluids over a stretching sheet: Passive control of nanoparticles at the surface, Can. J. Phys. 93 (2014) 725-733. doi:10.1139/cjp-2014-0370.

[57] Koriko OK, Adegbie KS, Shah NA, Animasaun I. L., \& Olotu MA. (2012) Numerical solutions of the partial differential equations for investigating the significance of partial slip due to lateral velocity and viscous dissipation: The case of blood-gold Carreau nanofluid and dusty fluid. Numerical Methods for Partial Differential Equations, in-press. 1-29. doi:10.1002/num.22754 
Figures

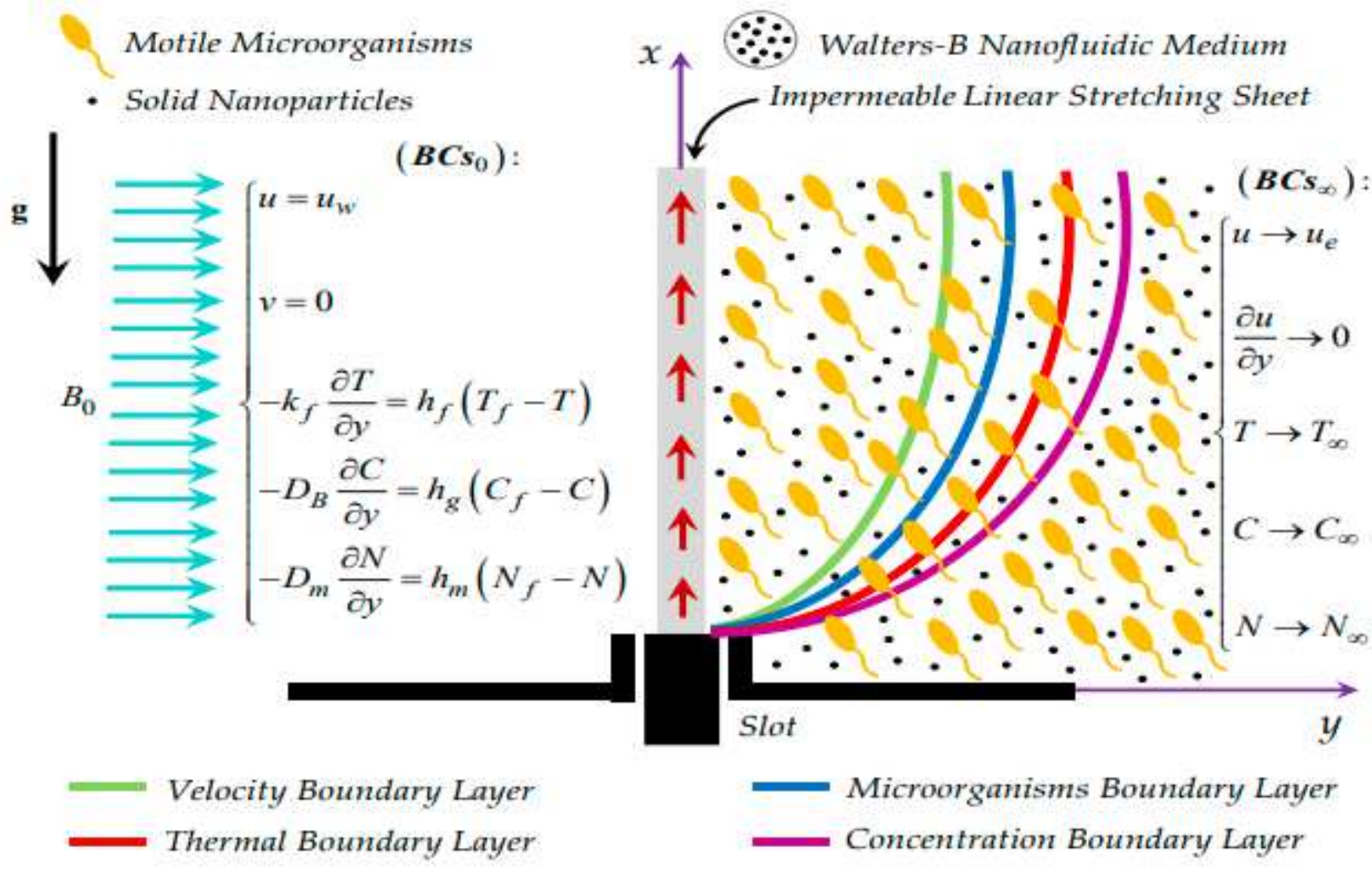

Figure 1

Geometrical configuration of the present non-Newtonian flow model.

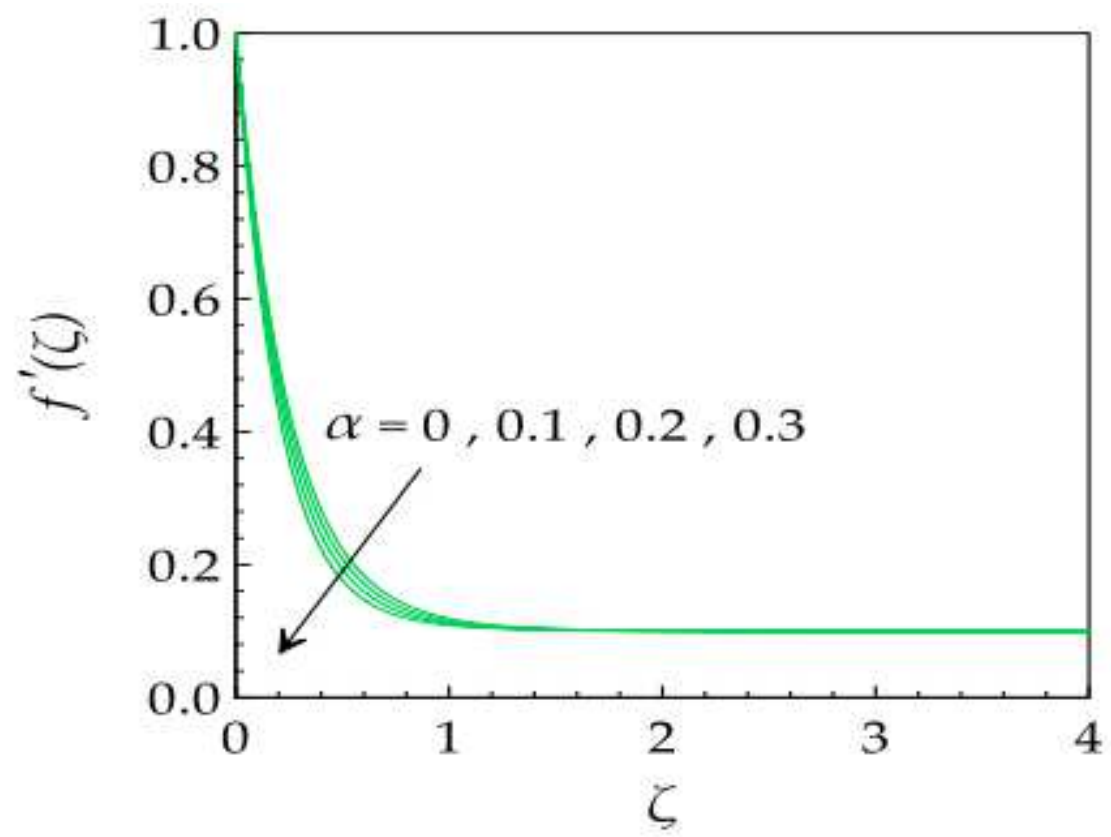

Figure 2

Please see the Manuscript PDF file for the complete figure caption 


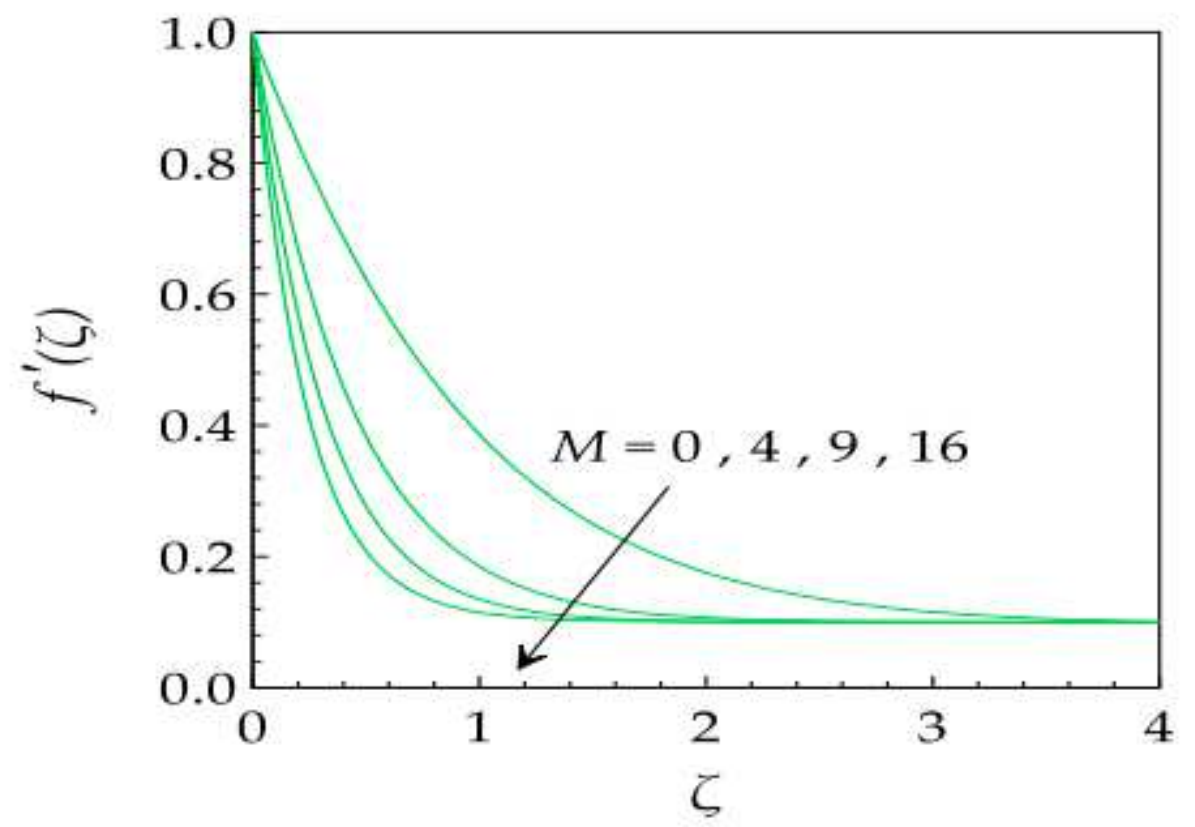

Figure 3

Please see the Manuscript PDF file for the complete figure caption

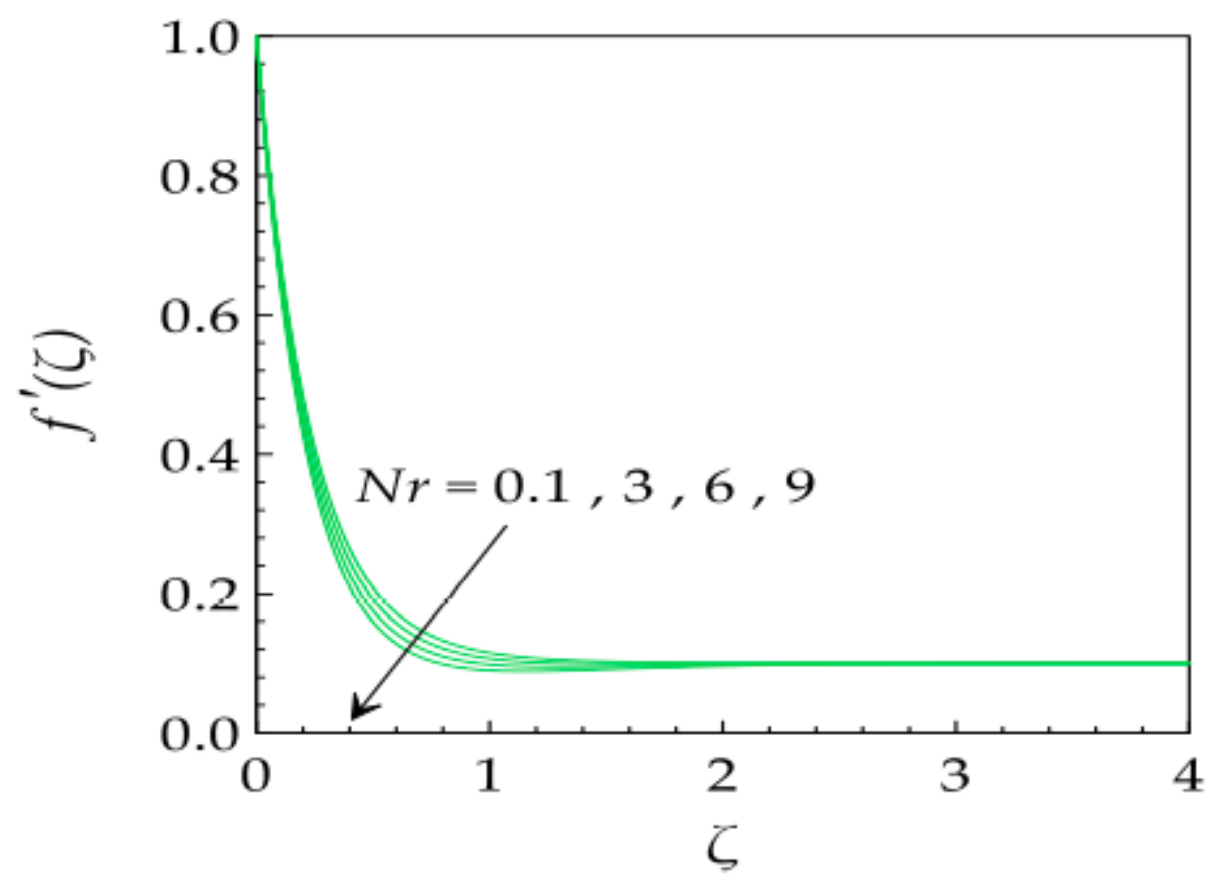

Figure 4

Please see the Manuscript PDF file for the complete figure caption 


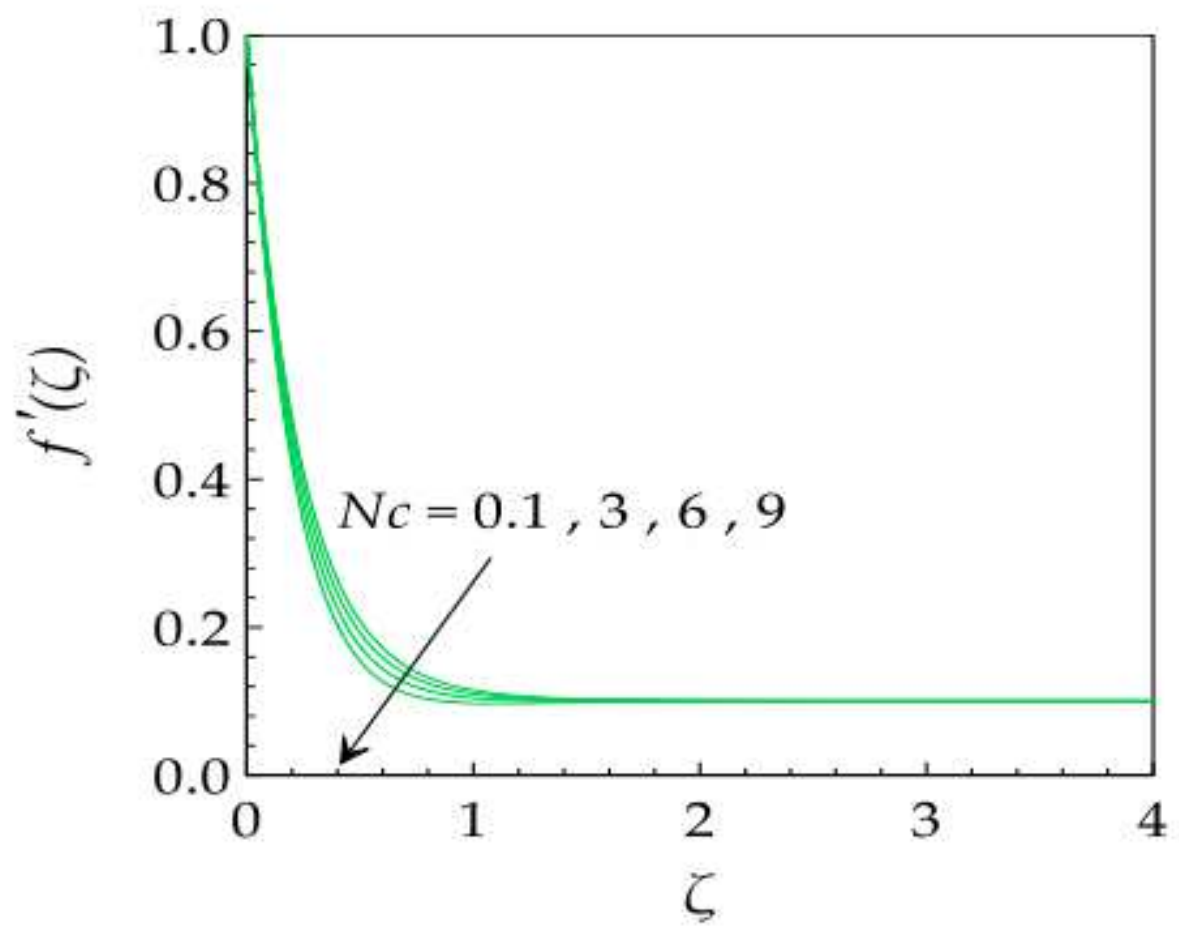

Figure 5

Please see the Manuscript PDF file for the complete figure caption

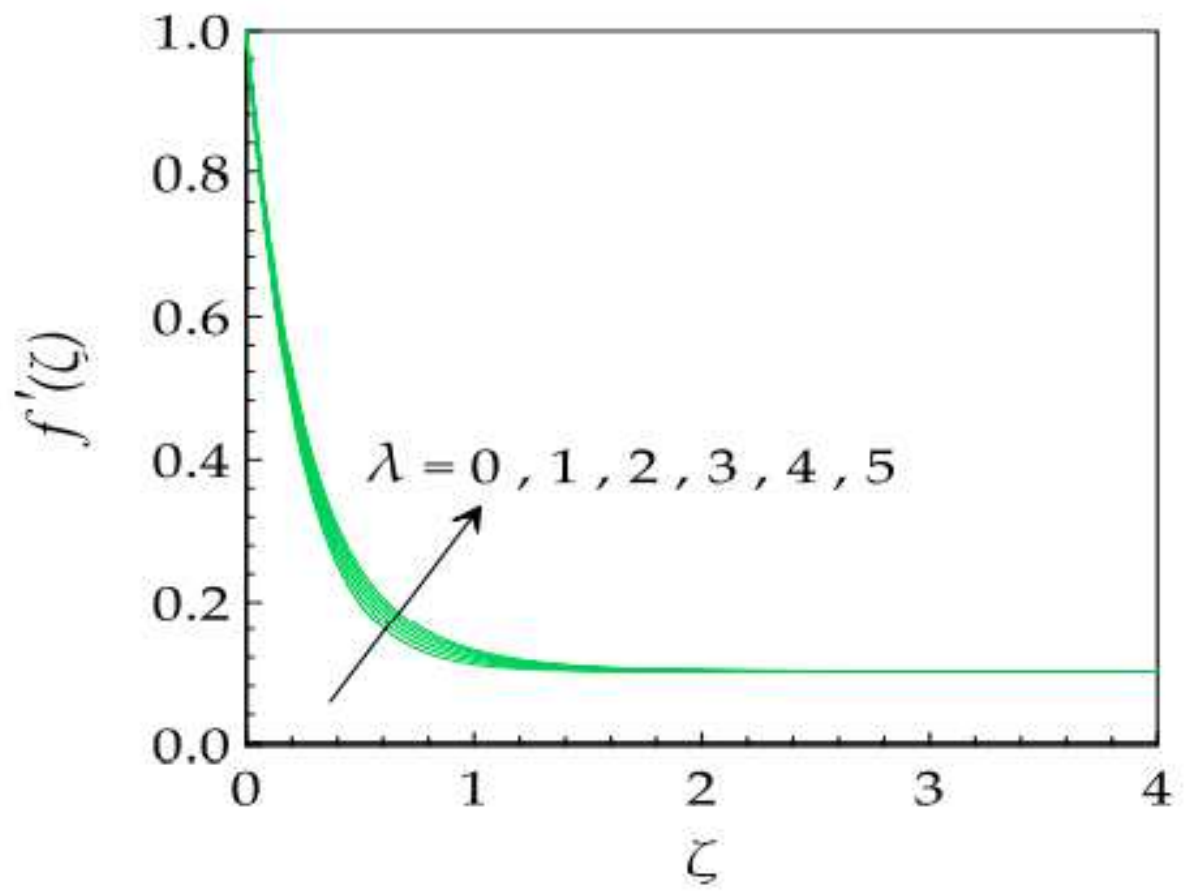

Figure 6

Please see the Manuscript PDF file for the complete figure caption 


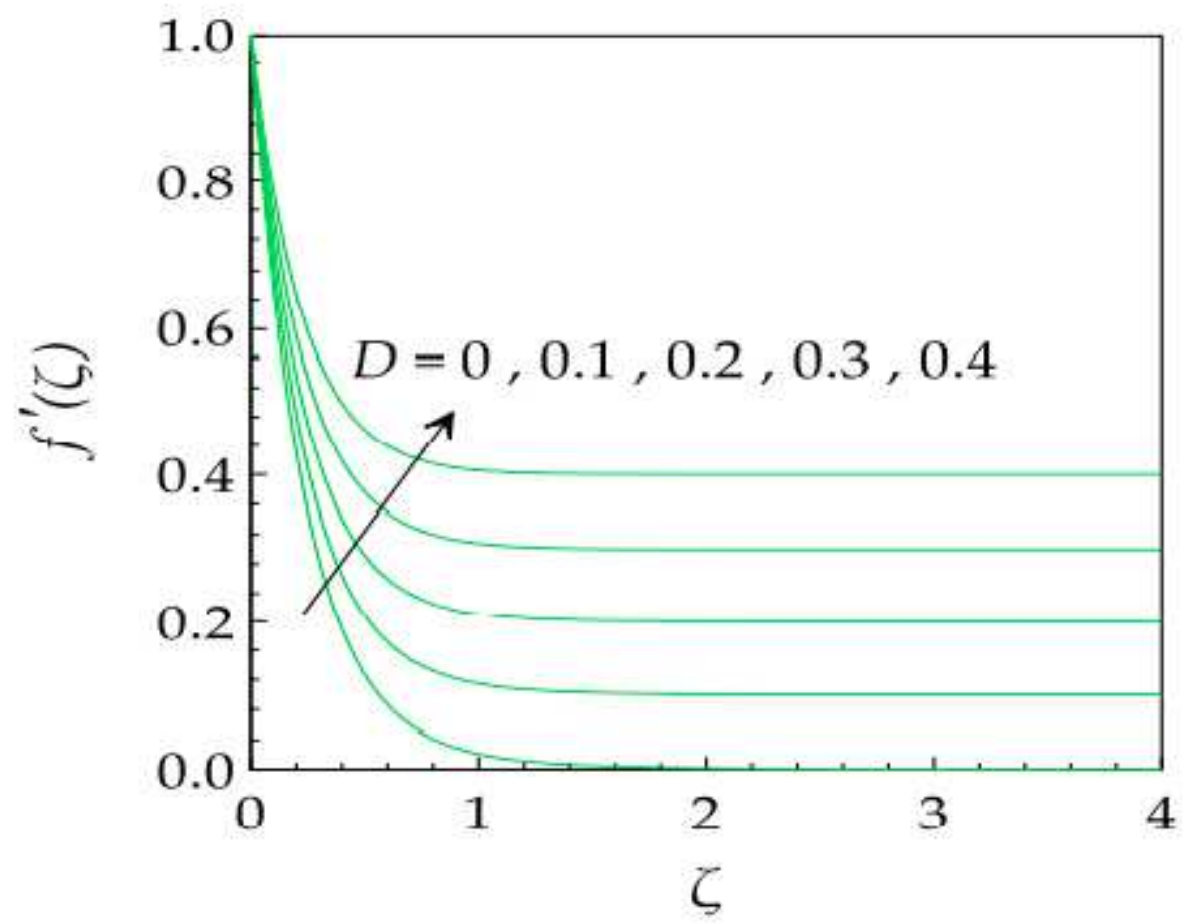

Figure 7

Please see the Manuscript PDF file for the complete figure caption
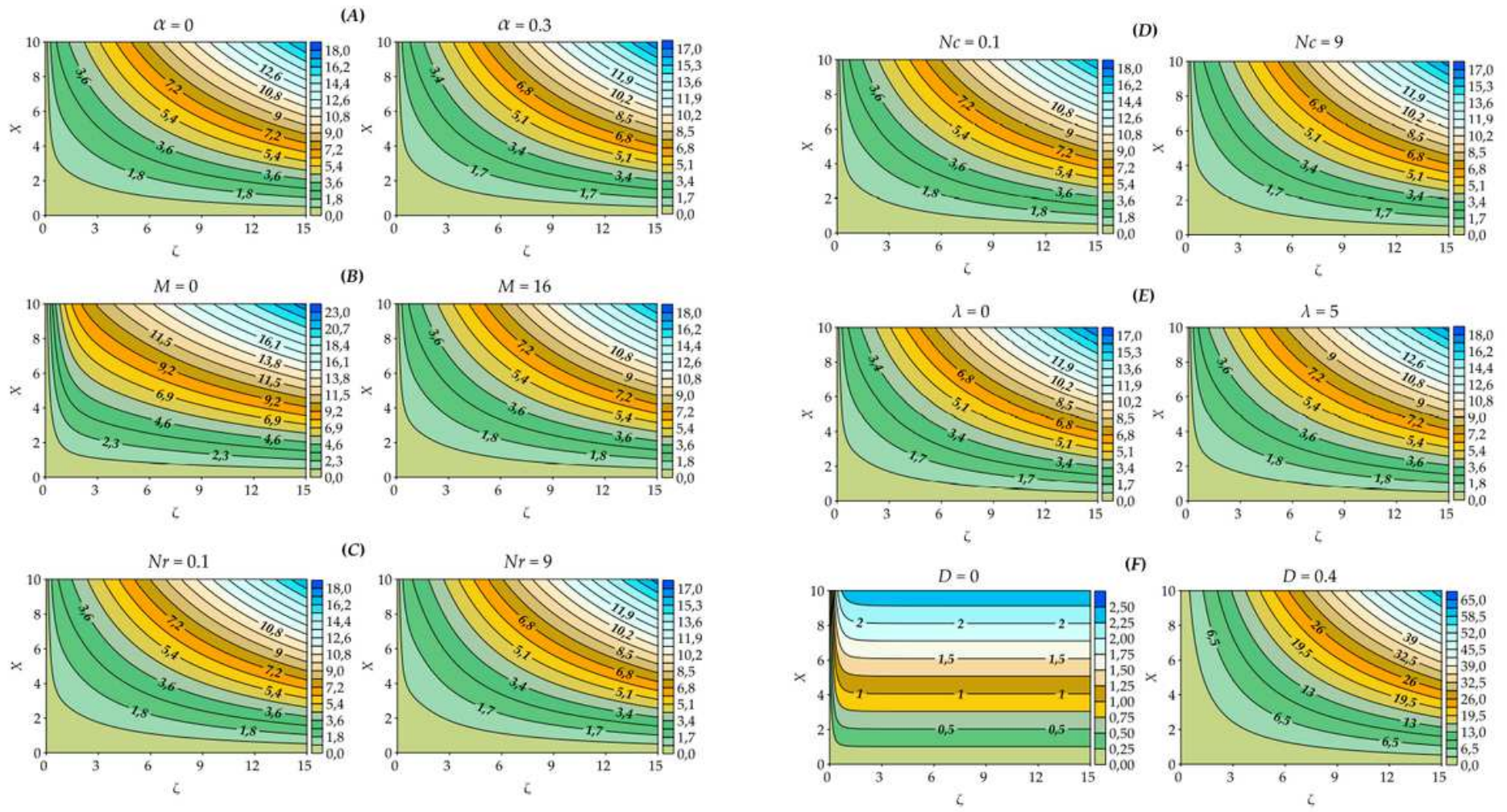

Figure 8

Please see the Manuscript PDF file for the complete figure caption 


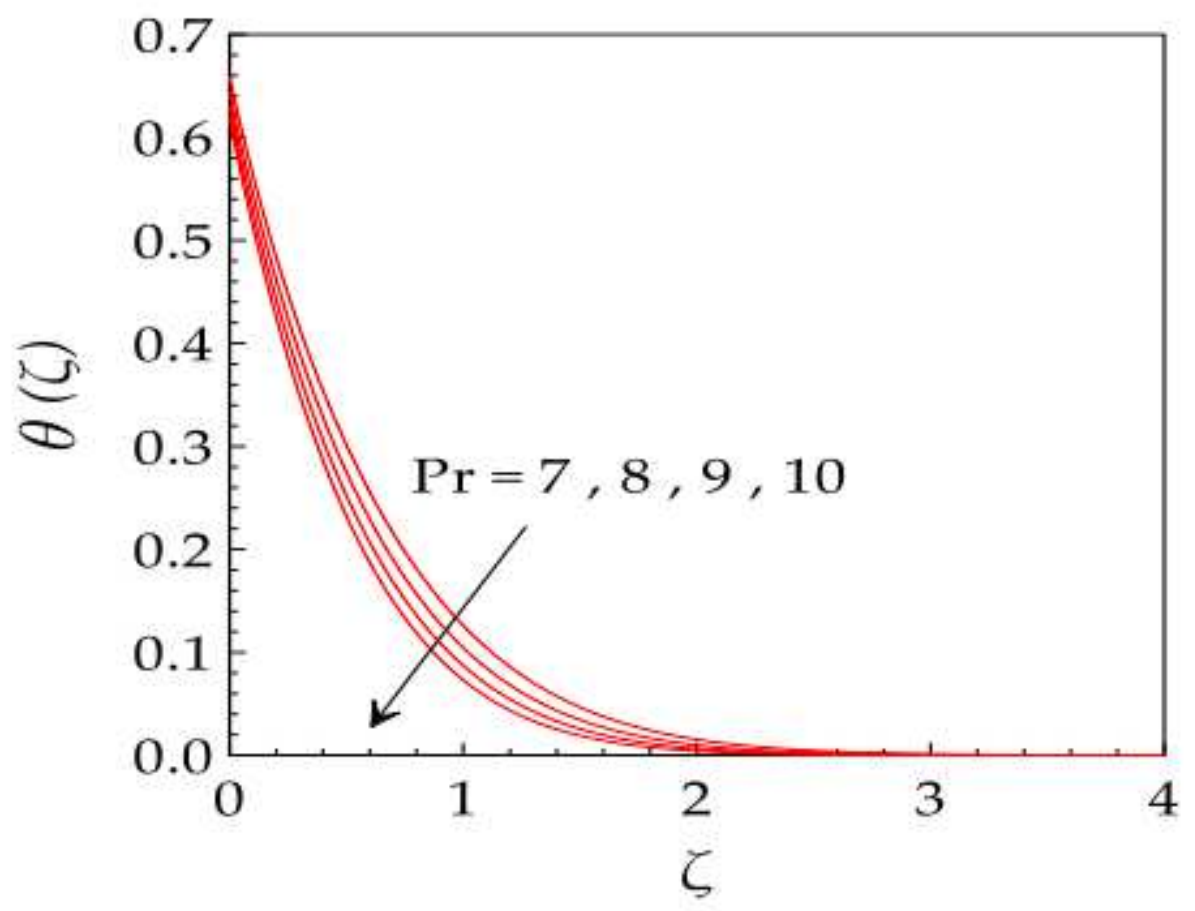

Figure 9

Please see the Manuscript PDF file for the complete figure caption

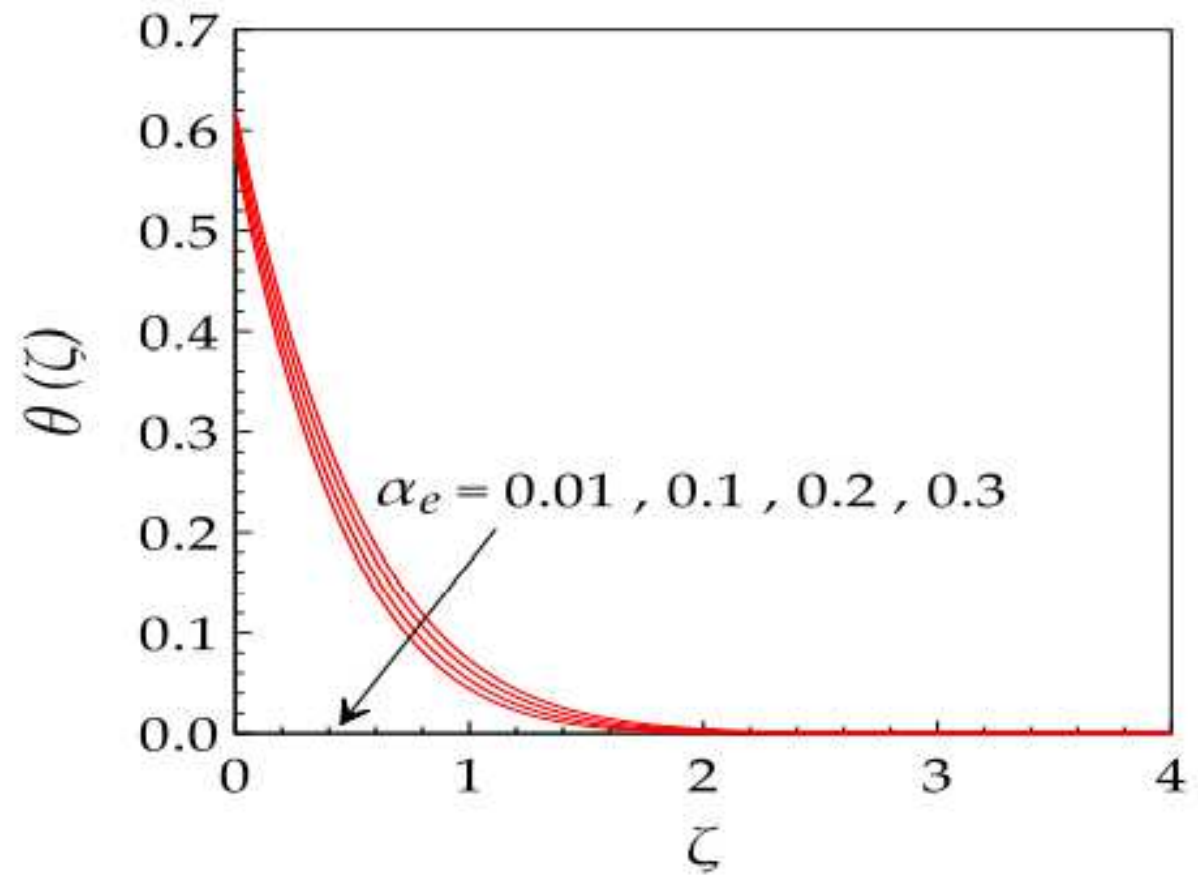

Figure 10

Please see the Manuscript PDF file for the complete figure caption 


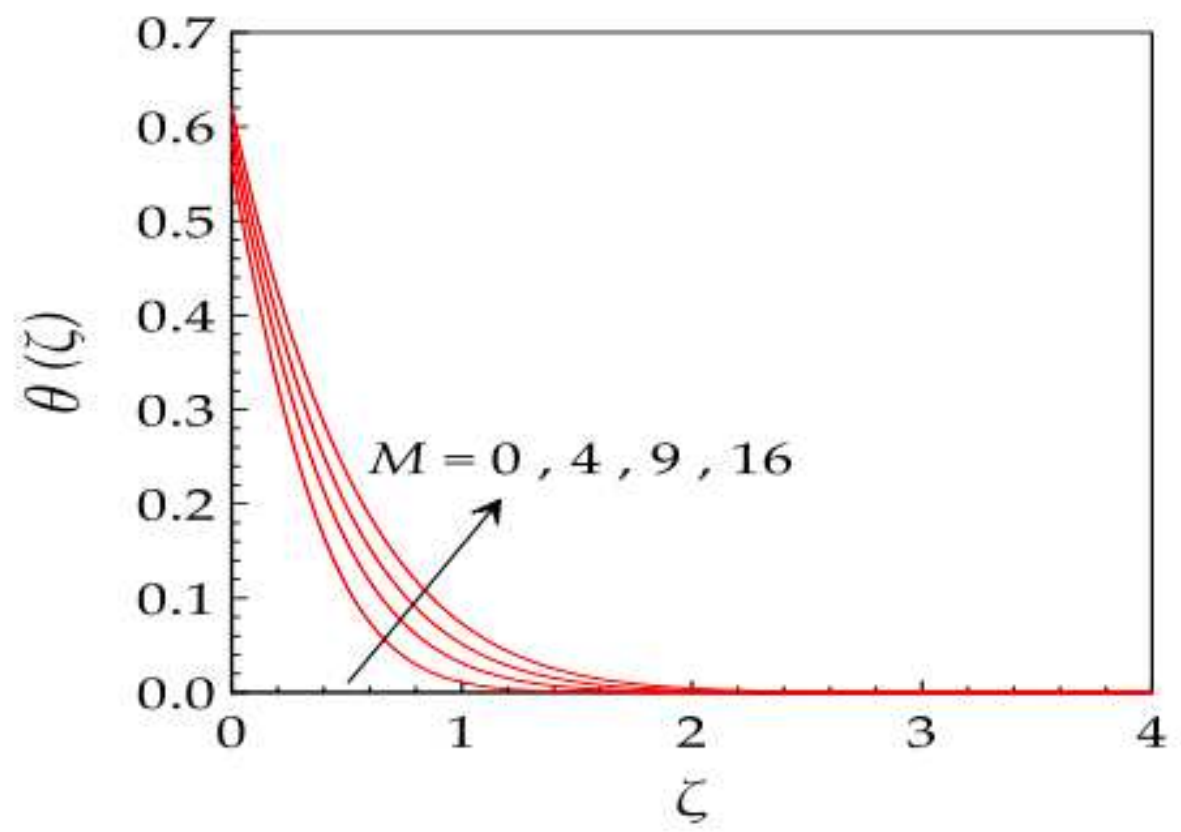

Figure 11

Please see the Manuscript PDF file for the complete figure caption

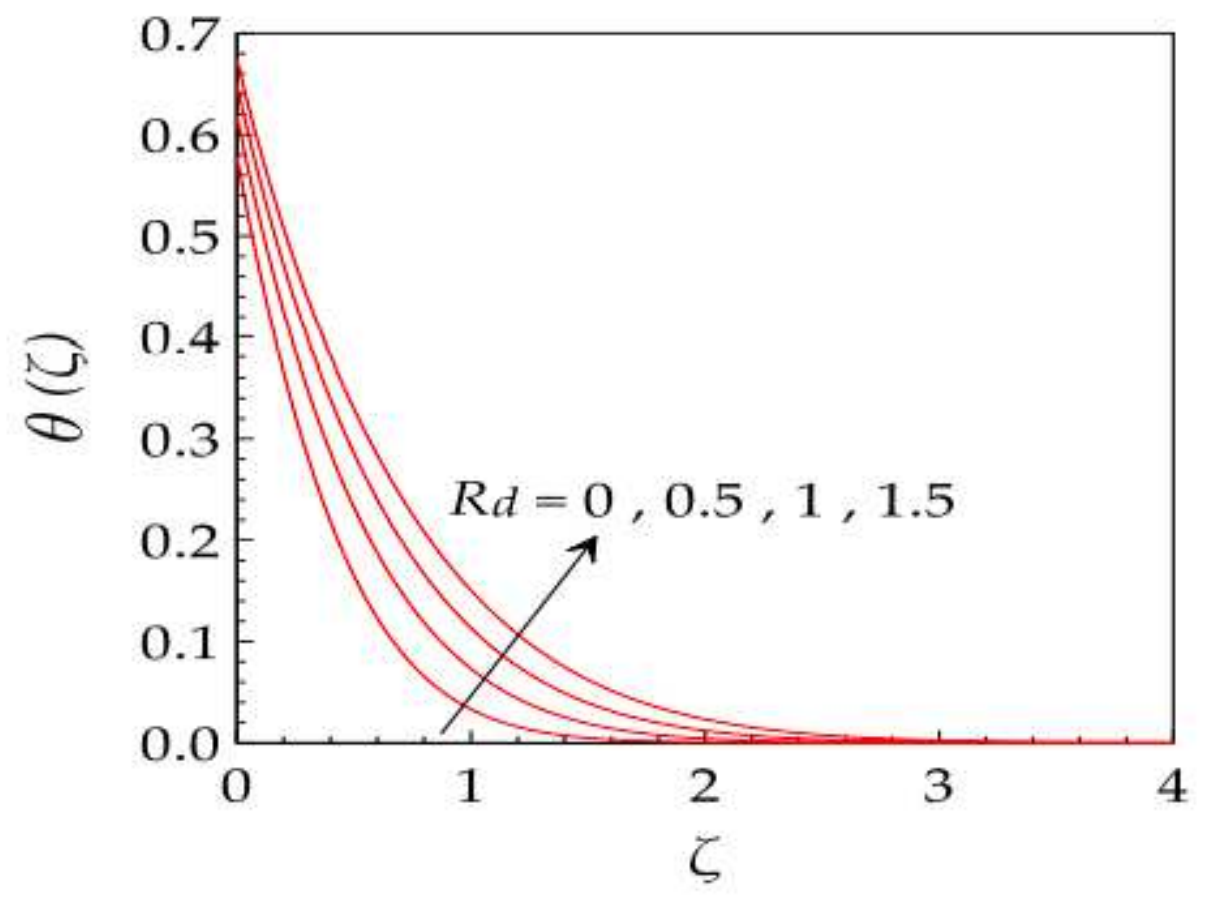

Figure 12

Please see the Manuscript PDF file for the complete figure caption 


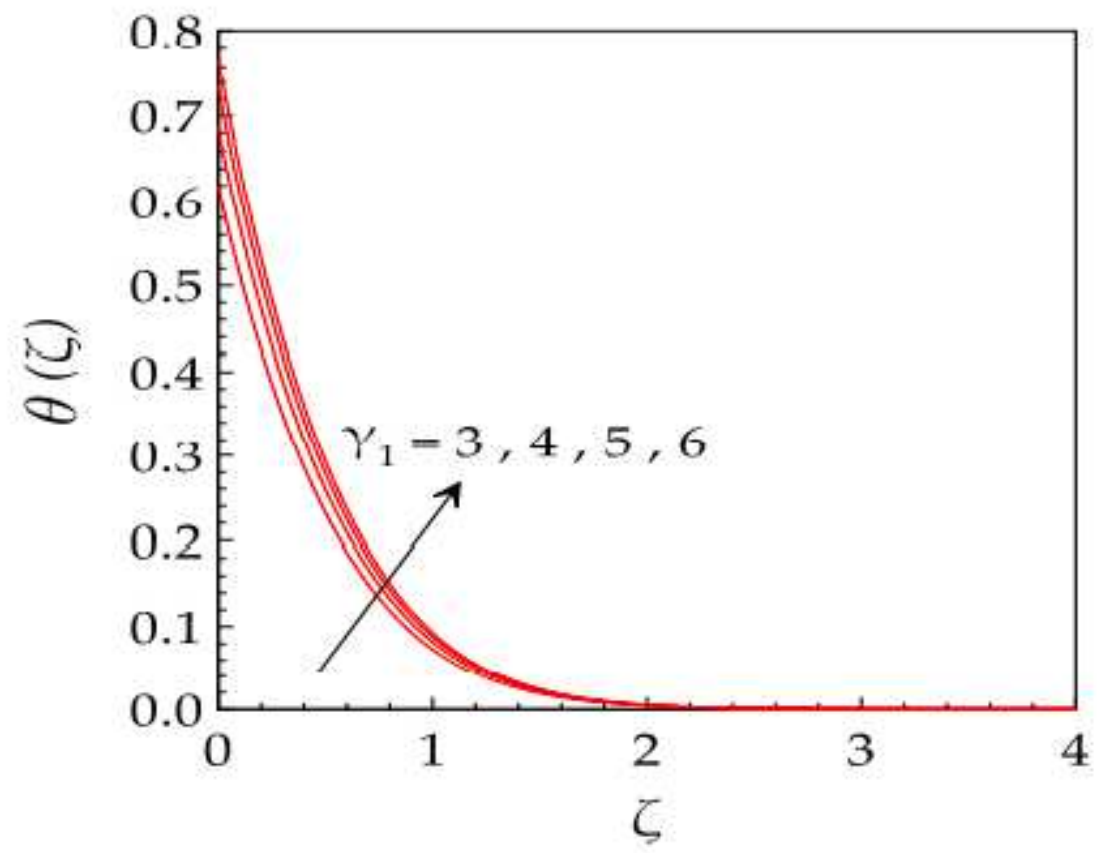

Figure 13

Please see the Manuscript PDF file for the complete figure caption

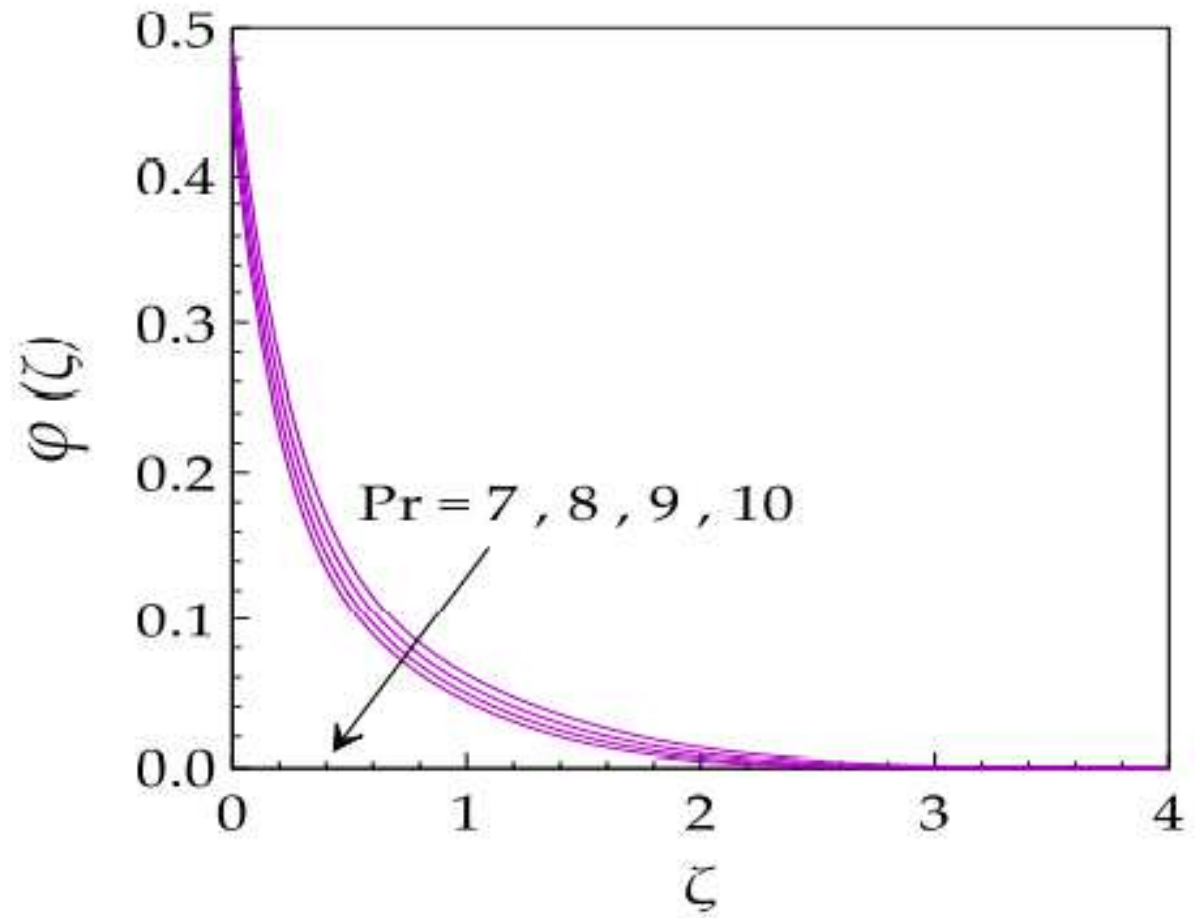

Figure 14

Please see the Manuscript PDF file for the complete figure caption 


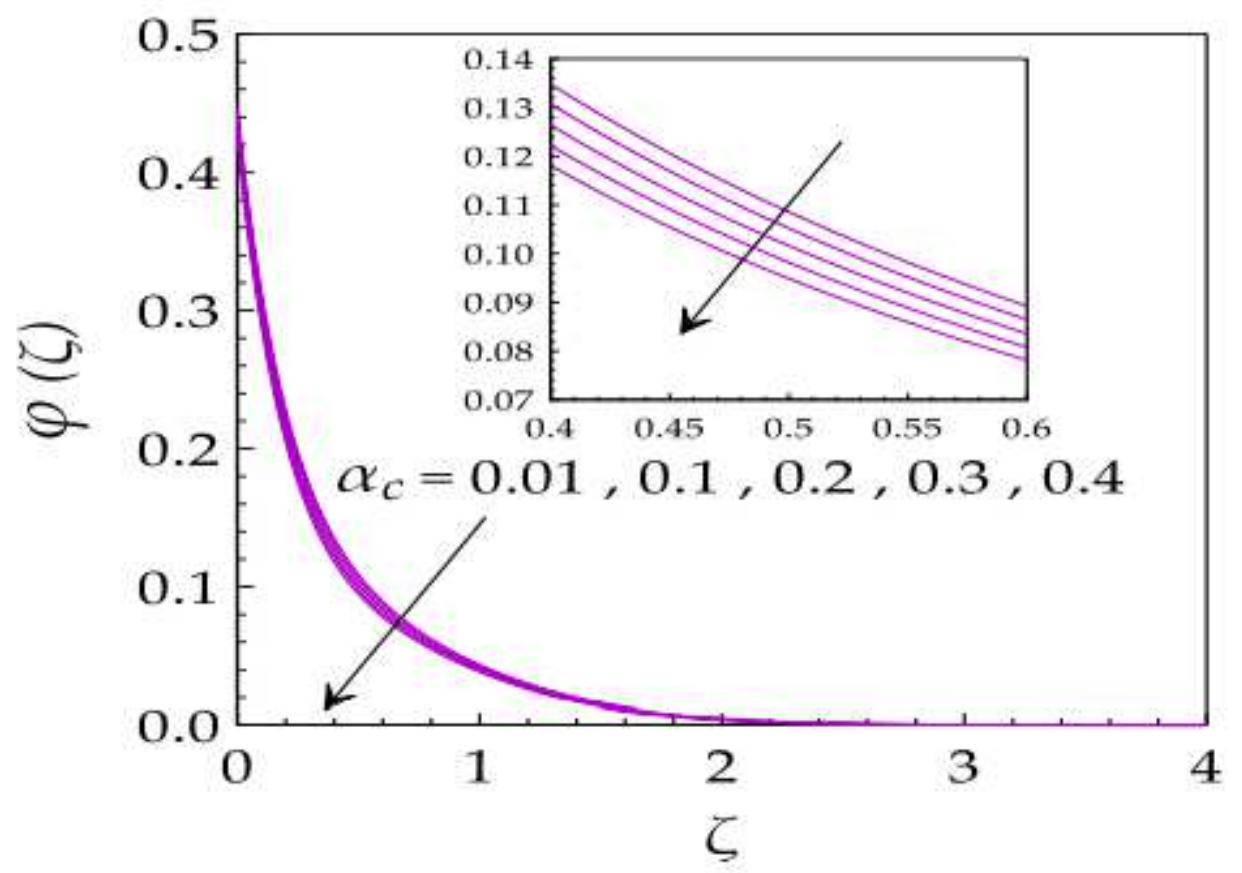

Figure 15

Please see the Manuscript PDF file for the complete figure caption

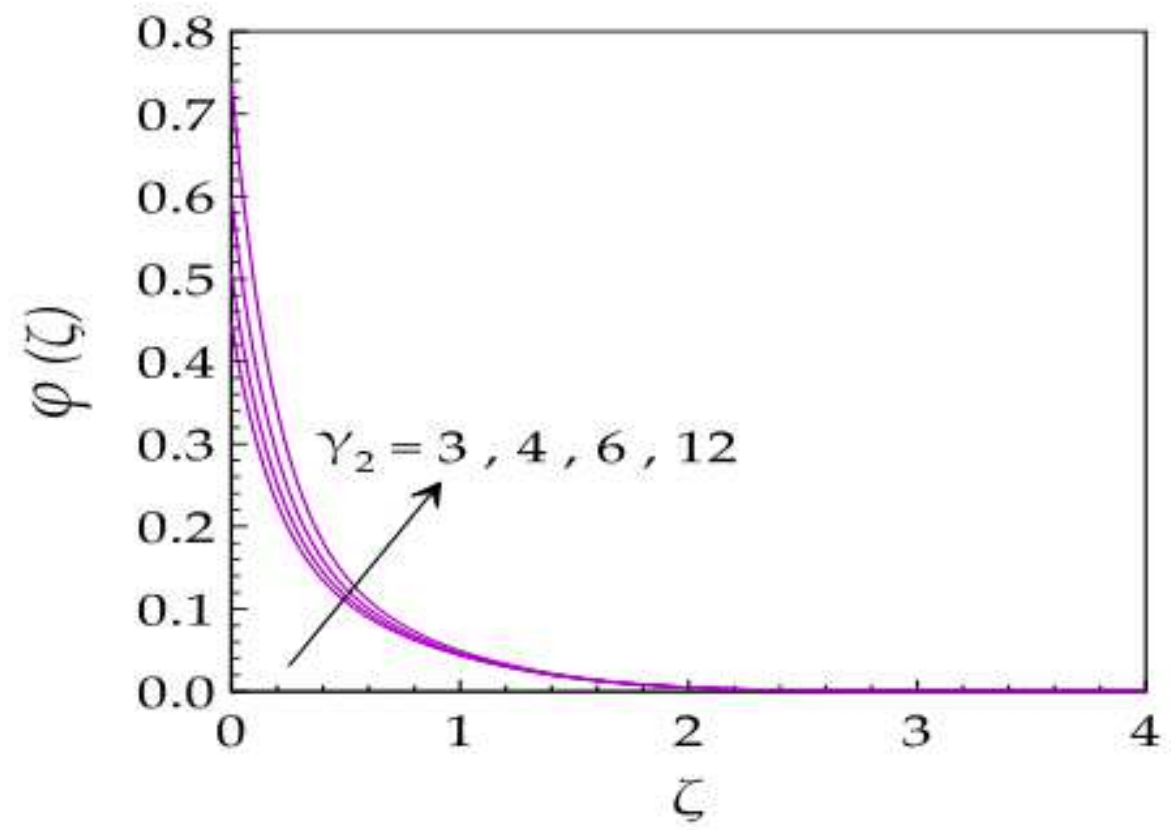

Figure 16

Please see the Manuscript PDF file for the complete figure caption 


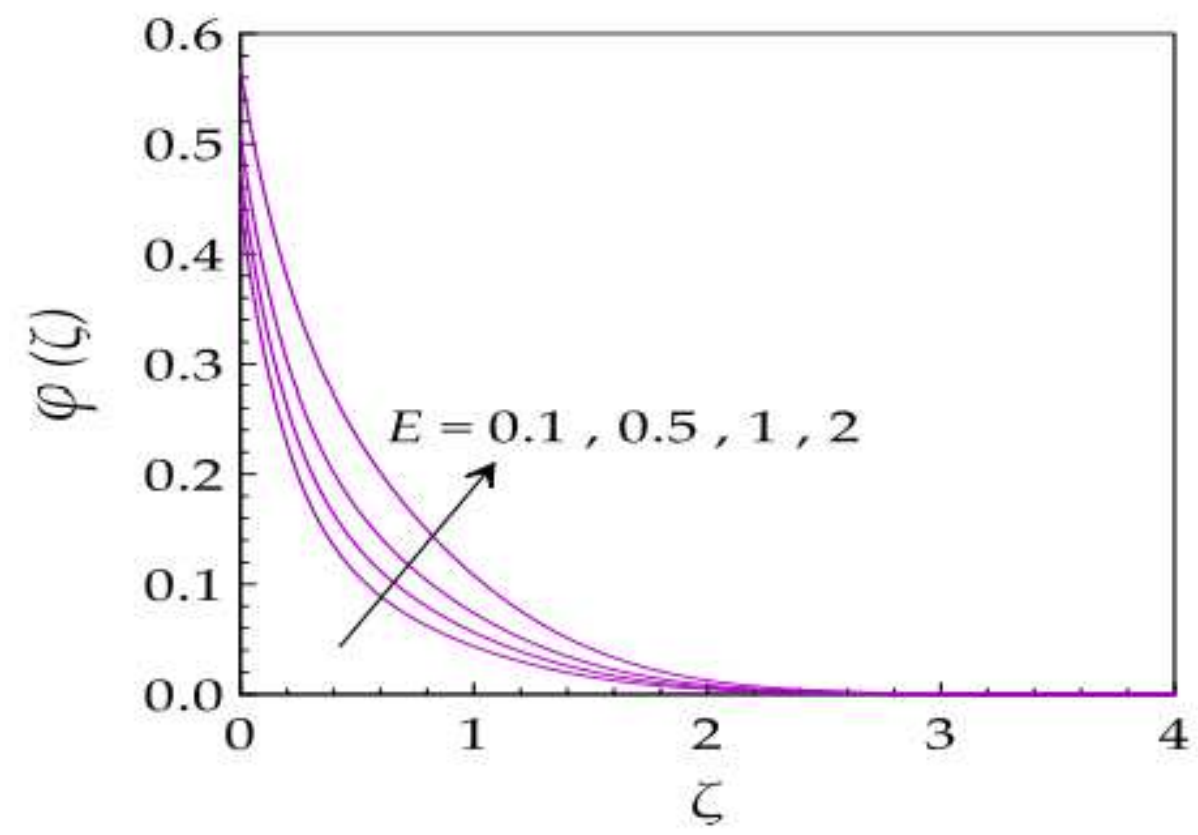

Figure 17

Please see the Manuscript PDF file for the complete figure caption

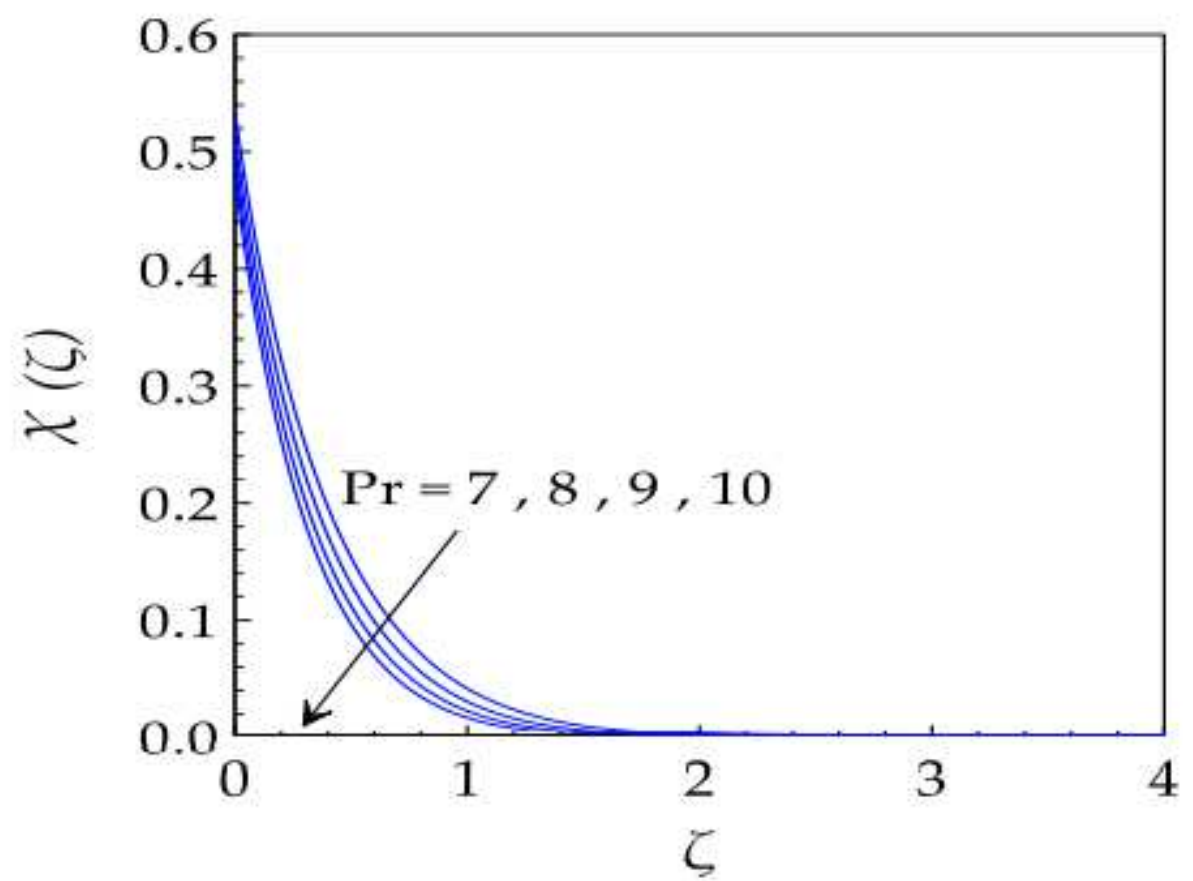

Figure 18

Please see the Manuscript PDF file for the complete figure caption 


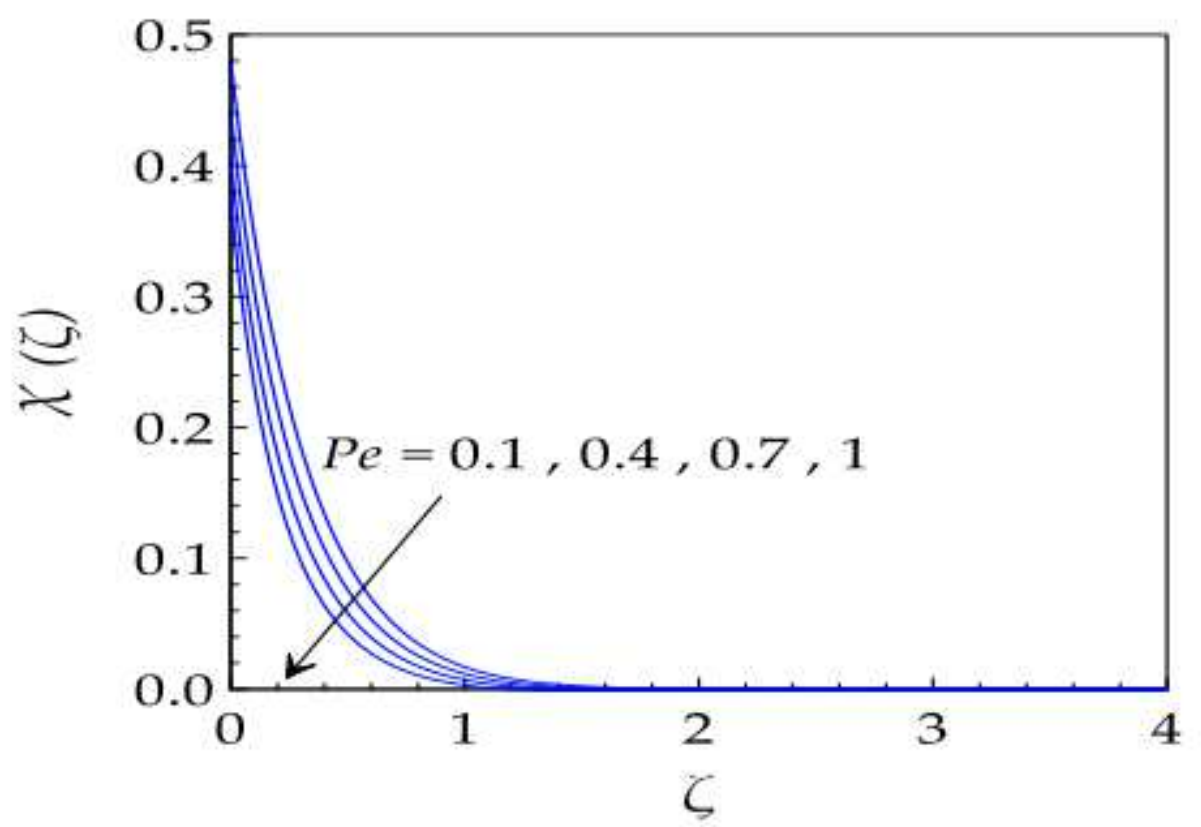

Figure 19

Please see the Manuscript PDF file for the complete figure caption

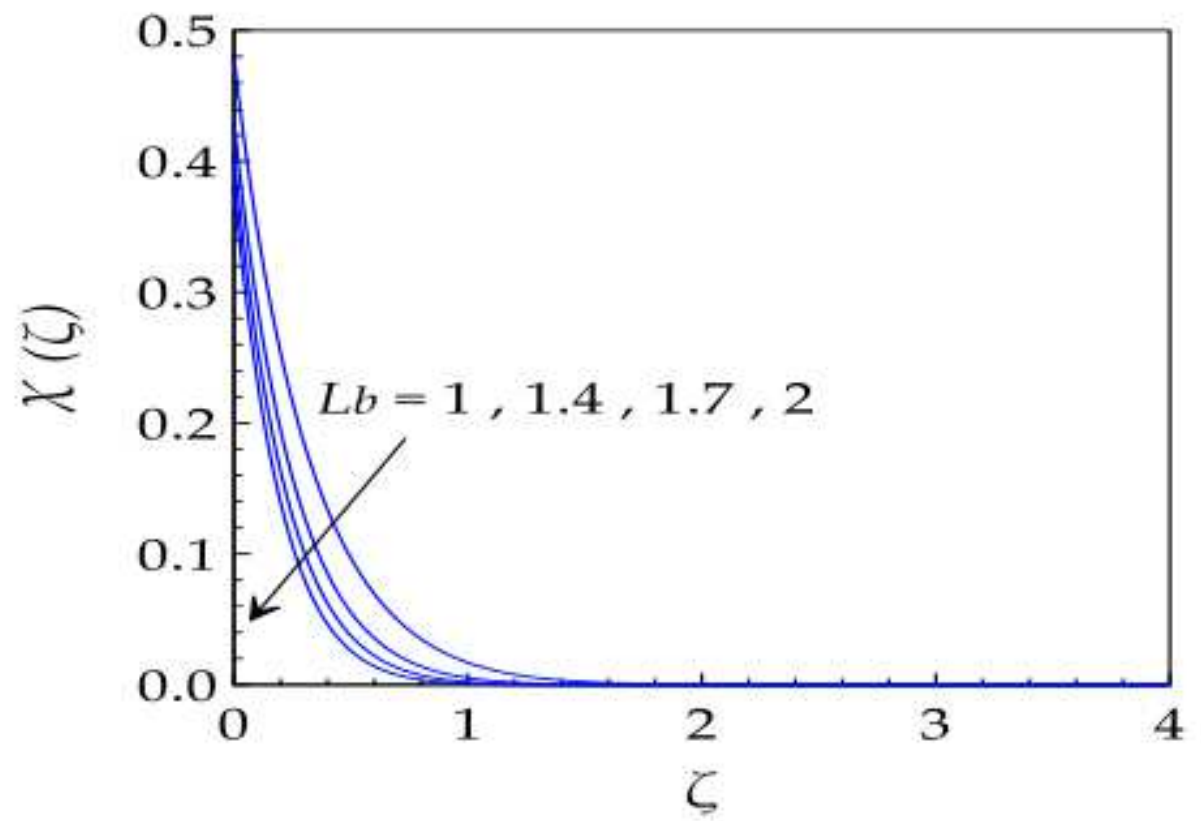

Figure 20

Please see the Manuscript PDF file for the complete figure caption 


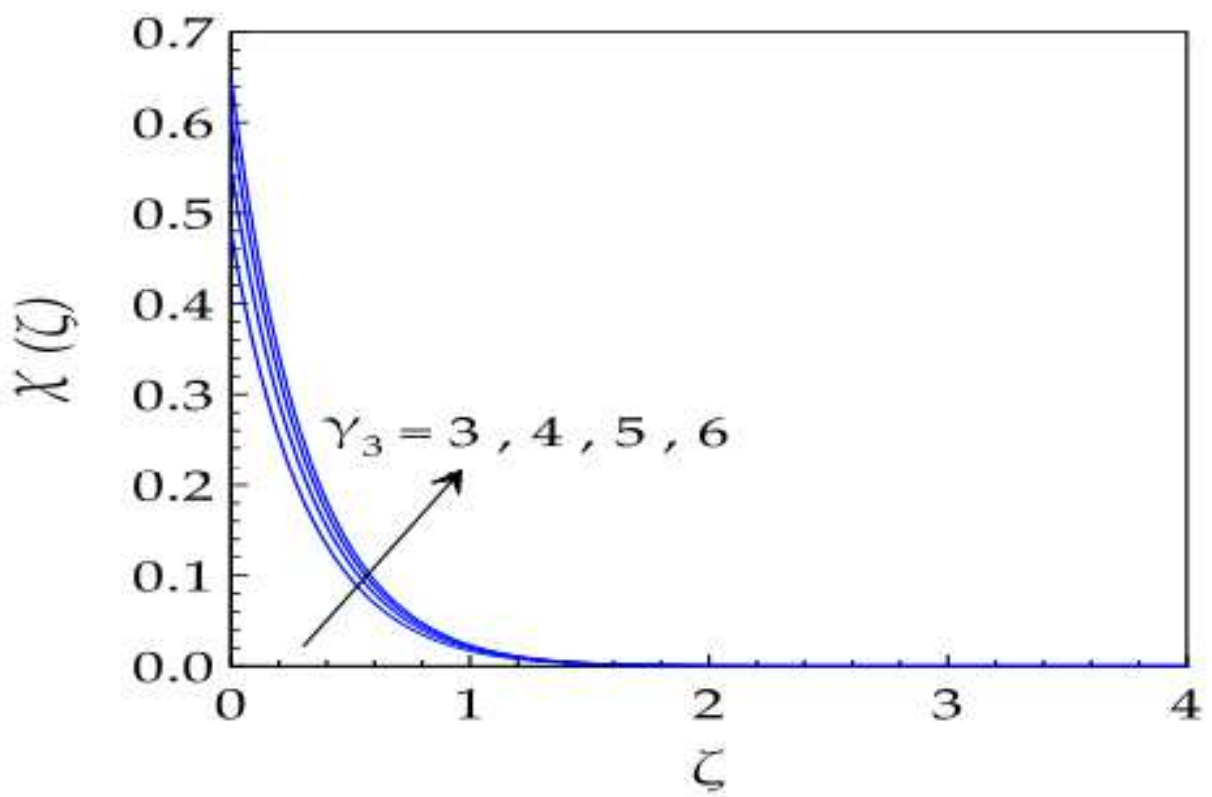

Figure 21

Please see the Manuscript PDF file for the complete figure caption 